UNIVERSITAT POLITÈCNICA DE VALENCIA

DEPARTAMENTO DE COMUNICACIONES

\title{
Few-Mode Transmission Technology for Ultra-High Capacity Optical Networks
}

\author{
Ph.D. THESIS \\ David García Rodríguez
}

Supervisors

Dr. Roberto Llorente Sáez

Dr. Juan Luis Corral González

Valencia, September 2018 



\section{Agradecimientos}

Deseo expresar mi agradecimiento a los directores de este trabajo Roberto Llorente Sáez y Juan Luis Corral González. Sin su apoyo, esfuerzo y dedicación el desarrollo de esta tesis doctoral no hubiera sido posible. El camino se recorre más fácilmente cuando te señalan en la dirección correcta.

También me gustaría dar las gracias a Maria Morant por sus consejos en aspectos técnicos así como la realización de los diferentes montajes experimentales.

Y no me olvido de algunos de mis compañeros de trabajo que más me han apoyado durante la realización de esta tesis doctoral, Marina Riquelme, Irene Olivares, Andrea Zanzi, SergioLuis Carrasco y Eduardo Valero. Gracias a Marina Riquelme por sus innumerables consejos en aspectos profesionales y motivación durante toda la tesis, a Irene Olivares por sus rápidos consejos en Matlab y algunas correcciones de inglés, a Andrea Zanzi por sus bromas y risas, y finalmente a Sergio Carrasco y Eduardo Valero por sus risas durante las quedadas en los cumpleaños.

Por último, me gustaría agradecer el apoyo de toda mi familia y amigos por la comprensión durante mi ausencia y trabajo todos estos años. 


\section{ABSTRACT}

Actual SMF networks are exclusively operated in the infra-red and $C+L$ bands (wavelengths in the range of $1300-1600 \mathrm{~nm}$ ) due to the commercial availability of amplification devices, such as erbium doped fiber amplifier (EDFA). However, the useable capacity in the infra-red band, although substantial, is finite and the unprecedented demand on that capacity means it is diminishing at an ever increasing rate. Few-mode propagation has recently emerged as a promising approach to maximize optical transmission capacity (bitrate $\times$ number of users). Thus, several techniques based on mode division multiplexing (MDM) using standard single-mode fibers (SSMFs) or optical waveguides in silicon-on-insulator (SOI) technology would suppose an increase of the bitrate to a very low cost.

In this Ph.D. thesis, different mode coupling and mode conversion techniques with the aim to increase the transport capacity in telecommunications systems over optical fiber are proposed. Concretely, the main aim is the development of the technology to achieve MDM using a limited controlled number of modes. Two different MDM scenarios based on two distinct wavelengths have been considered. On one hand, using the $850 \mathrm{~nm}$ wavelength over SSMF favors the use of optical and electro-optical devices with costs much lower than their equivalent in the $\mathrm{C}+\mathrm{L}$ band. This novel transmission technology enables a new generation of very high capacity optical interconnections applicable to chip-to-chip links, to optical backplanes, and also to high-performance computing clusters and network switching centre interconnections. On the other hand, using the $1550 \mathrm{~nm}$ wavelength over optical waveguides based on SOI, i.e., Si (Silicon) above $\mathrm{SiO}_{2}$ substrate (silicon oxide), allows the use of integrated devices offering a less size, better repeatability and robustness in comparison with the optical fiber devices.

Fused fiber couplers are proposed as key elements to (de)multiplex different fiber modes in a MDM link at $850 \mathrm{~nm}$. The use of a symmetric directional coupler (DC) as a (de)multiplexer requires the use of an additional mode converter. The use of an asymmetrical directional coupler (ADC) as optical (de)multiplexer and mode converter is proposed, avoiding the necessity of an additional mode converter and simplifying the MDM scheme.

Furthermore, in this Ph.D. thesis it is also proposed and evaluated the design of a mechanical mode converter at $850 \mathrm{~nm}$ using a SSMF. This technique permits to obtain the first high order mode with high quality and without the necessity of using an ADC.

After that, it is analyzed and investigated the employment of commercial optical couplers (designed at $1550 \mathrm{~nm}$ ) at $850 \mathrm{~nm}$ wavelength operation, thus avoiding the use of optical couplers and mode converters specifically designed at $850 \mathrm{~nm}$ wavelength. The MDM system costs are reduced as fewer devices are required and commercial components designed at $1550 \mathrm{~nm}$ are cheaper than the counterparts at $850 \mathrm{~nm}$.

In this Ph.D. thesis it is also considered the employment of ADCs over strip waveguides based on SOI technology for the conversion and multiplexing of the optical modes, from single-mode waveguide to high order mode waveguide at the $1550 \mathrm{~nm}$ wavelength. Thus, it has been studied and experimentally investigated different designs aimed to achieve the most robust configuration, in which the yield is less affected by the fabrication tolerances.

Furthermore, the use of DCs over ridge waveguides is commonly employed and it offers better performance than strip waveguides. For this reason, the Ph.D. thesis studies and evaluates the use of ADCs with ridge waveguides by considering the effective refractive indexes of the even and odd supermodes analysis. In this way, a comparison between strip and ridge structures is done in order to find the optimum design that offer the best features.

Finally, it is analyzed the design of a grating coupler capable of multiplexing and demultiplexing the fundamental and the high order mode from the waveguide to the optical fiber and vice versa. Thus, different designs are evaluated in order to achieve a design more robust and efficient to the coupling misalignments.

The use of all techniques will allow to perform the modal multiplexing on the different scenarios and thus increase the bandwidth of the system. 


\section{RESUMEN}

Actualmente únicamente se utiliza la banda infra-roja y la banda $C+L$ (longitudes de onda de $1300 \mathrm{~nm}$ a $1600 \mathrm{~nm}$ ). Esto es debido a la gran disponibilidad comercial de dispositivos de amplificación, principalmente amplificadores ópticos de fibra dopada con erbio (EDFA, del inglés erbium-doped fiber amplifier), en dicha banda. La capacidad útil de la banda $\mathrm{C}+\mathrm{L}$, aunque sustancial, es finita, y se está agotando debido a la creciente demanda de capacidad exigida por los usuarios. La propagación de pocos modos (FMF, del inglés few-mode fiber) en la fibra óptica ha surgido como una oportunidad tecnológica para incrementar la capacidad de transmisión (régimen binario $\times$ número de usuarios) de los enlaces ópticos. Por ello, la utilización de técnicas de multiplexación por división de modos (MDM, del inglés mode division multiplexing) en fibras monomodo estándar (SSMF, del inglés standard single-mode fiber), ampliamente desarrollada en redes ópticas comerciales y de menor coste, o en guías ópticas basadas en SOI (del inglés, silicon-on-insulator), dada su fácil fabricación, supondría un incremento del régimen binario a un coste muy reducido.

En esta Tesis Doctoral, se propone diferentes técnicas de acoplo y conversión modal destinadas a aumentar la capacidad de transporte en sistemas de telecomunicaciones sobre fibra óptica. En particular, el objetivo principal es el desarrollo de la tecnología necesaria para conseguir una multiplexación modal utilizando un número limitado de modos, de manera controlada. Para ello, se estudian dos escenarios MDM con dos longitudes de onda distinta. Por un lado, usando la longitud de onda de $850 \mathrm{~nm}$ sobre SSMF favoreciendo la utilización de componentes ópticos y electro-ópticos de coste mucho menor que sus equivalentes en la banda C+L. Esta novedosa tecnología de transmisión permitirá una nueva generación de interconexiones ópticas de muy alta capacidad aplicable a enlaces chip-a-chip, a backplanes ópticos y también a clústeres de computación de altas prestaciones y centros de conmutación de red. Por otro lado, usando la longitud de onda de $1550 \mathrm{~nm}$ sobre guías ópticas basadas en SOI, es decir, Si (silicio) sobre sustrato de $\mathrm{SiO}_{2}$ (óxido de silicio) favoreciendo la utilización de dispositivos basados en tecnología integrada que ofrecen un menor tamaño, mejor repetibilidad y robustez que los dispositivos basados en fibra óptica.

Para ello, se propone el uso de acopladores ópticos fusionados siendo un elemento indispensable a la hora de multiplexar y demultiplexar los distintos modos ópticos en un enlace MDM a $850 \mathrm{~nm}$. Esta técnica permite multiplexar/demultiplexar los modos ópticos cuando el tipo de acoplador óptico utilizado es simétrico (DC, del inglés directional coupler), siendo necesario la utilización de un conversor de modos. También se estudia la posibilidad de convertir el modo óptico mediante la utilización de un acoplador óptico asimétrico (ADC, del inglés asymmetrical directional coupler), no siendo necesario utilizar un conversor de modos y simplificando el esquema MDM.

Además, en esta tesis doctoral también se propone y evalúa el diseño de un conversor de modos mecánico basado en SSMF. Esta técnica permite obtener el primer modo de orden superior con una alta calidad y sin la necesidad de utilizar un ADC.

Después de esto, se propone y evalúa la posibilidad de utilizar acopladores comerciales (diseñados a $1550 \mathrm{~nm}$ ) a la longitud de onda de $850 \mathrm{~nm}$ permitiendo de esta forma reducir la necesidad de utilizar acopladores ópticos y conversores modales específicamente diseñados en dicha longitud de onda. Esta técnica reduciría los costes del sistema al necesitar un menor número de dispositivos y aprovechar los dispositivos diseñados a $1550 \mathrm{~nm}$, siendo más económicos que los diseñados a $850 \mathrm{~nm}$.

En esta Tesis también se propone el uso de ADCs en guías strip basadas en SOI para la conversión y multiplexación de los modos ópticos desde la guia fundamental a la guia de dos modos, a la longitud de onda de $1550 \mathrm{~nm}$. Para ello se estudia y demuestra experimentalmente diferentes diseños con el fin de obtener el diseño más robusto frente a las tolerancias de fabricación consiguiendo un rendimiento óptimo.

Además, el uso de DCs sobre guías ridge es comúnmente utilizado y ofrece mejores prestaciones que el basado en guías strip, por ese motivo esta Tesis estudia y evalúa el uso 
de ADCs sobre guías ridge mediante el método de análisis de los índices efectivos de los supermodos par e impar. De esta forma se realiza una comparación entre los diseños óptimos de ambas estructuras (strip y ridge) con el objetivo de averiguar qué diseño ofrece mejores prestaciones.

Por último, se propone y estudia el diseño de un acoplador grating capaz de multiplexar y demultiplexar los modos ópticos del modo fundamental y del primer orden superior desde la guia óptica a la fibra óptica y viceversa. Para ello se proponen diferentes diseños con el objetivo de conseguir un diseño más tolerante y eficiente frente a los errores por desalineamiento obteniendo un acoplo óptimo.

El uso de todas estas técnicas permitirá realizar la multiplexación modal sobre los diferentes escenarios y así aumentar el ancho de banda del sistema. 


\section{RESUM}

Actualment únicament s'utilitza la banda infraroja i la banda $C+L$ (longituds d'ona de $1300 \mathrm{~nm}$ a $1600 \mathrm{~nm}$ ). Açò és degut a la gran disponibilitat comercial de dispositius d'amplificació, principalment amplificadors òptics de fibra dopada amb erbi (EDFA, de l'anglès erbium-doped fiber amplifier), en dita banda. La capacitat útil de la banda $\mathrm{C}+\mathrm{L}$, encara que substancial, és finita, i s'està esgotant a causa de la creixent demanda de capacitat exigida pels usuaris. La propagació de pocs modes (FMF, de l'anglès few-mode fiber) en la fibra òptica ha sorgit com una oportunitat tecnològica per a incrementar la capacitat de transmissió (règim binari $x$ nombre d'usuaris) dels enllaços òptics. Per això, la utilització de tècniques de multiplexació per divisió de modes (MDM, de I'anglès mode division multiplexing) en fibres monomodo estàndard (SSMF, de l'anglès standard single-mode fiber), àmpliament desenrotllada en xarxes òptiques comercials i de menor cost, $\mathrm{O}$ en guies òptiques basades en SOI (de l'anglès, silicon-on-insulator), donada la seua fàcil fabricació, suposaria un increment del règim binari a un cost molt reduit.

En aquesta Tesi Doctoral, es proposen diferents tècniques d'acoblament i conversió modal destinades a augmentar la capacitat de transport en sistemes de telecomunicacions sobre fibra òptica. En particular, l'objectiu principal és el desenrotllament de la tecnologia necessària per a aconseguir una multiplexació modal utilitzant un número limitat de modes, de manera controlada. Per a això, s'estudien dos escenaris MDM amb dos longituds d'onda distinta. D'una banda, usant la longitud d'ona de $850 \mathrm{~nm}$ sobre SSMF afavorint la utilització de components òptics i electro-òptics de cost molt menor que els seus equivalents en la banda $\mathrm{C}+\mathrm{L}$. Aquesta nova tecnologia de transmissió permetrà una nova generació d'interconnexions òptiques de molt alta capacitat aplicable a enllaços chip-a-chip, a backplanes òptics i també a clústers de computació d'altes prestacions i centres de commutació de xarxa. D'altra banda, usant la longitud d'ona de $1550 \mathrm{~nm}$ sobre guies òptiques basades en SOI, és a dir, Si (silici) sobre substrat de $\mathrm{SiO}_{2}$ (òxid de silici) afavorint la utilització de dispositius basats en tecnologia integrada que ofereixen una menor grandària, millor repetibilitat i robustesa que els dispositius basats en fibra òptica.

Per a això, es proposa l'ús d'acobladors òptics fusionats sent un element indispensable a l'hora de multiplexar i demultiplexar els distints modes òptics en un enllaç MDM. Aquesta tècnica permet multiplexar/demultiplexar els modes òptics quan el tipus d'acoblador òptic utilitzat és simètric (DC, de l'anglès directional coupler), sent necessari la utilització d'un convertidor de modes. També s'estudia la possibilitat de convertir el mode òptic per mitjà de la utilització d'un acoblador òptic asimètric ( $A D C$, de l'anglès asymmetrical directional coupler), no sent necessari utilitzar un convertidor de modes i simplificant l'esquema MDM.

Es mes, en aquesta tesi doctoral també es proposa i avalua el disseny d'un convertidor de modes mecànic basat en SSMF. Aquesta tècnica permet obtindre el primer mode d'orde superior amb una alta qualitat sense la necessitat d'utilitzar un ADC.

Després d'açò, es proposa i avalua la possibilitat d'utilitzar acobladors comercials (dissenyats a $1550 \mathrm{~nm}$ ) a la longitud d'ona de $850 \mathrm{~nm}$ permetent d'esta manera reduir la necessitat d'utilitzar acobladors òptics i convertidors modals específicament dissenyats en la dita longitud d'ona. Aquesta tècnica reduiria els costos del sistema al necessitar un menor nombre de dispositius i aprofitant els dispositius dissenyats a $1550 \mathrm{~nm}$, sent més econòmics que els dissenyats a $850 \mathrm{~nm}$.

En aquesta Tesi també es proposa l'ús de ADCs en guies strip basades en SOI per a la conversió i multiplexació dels modes òptics des de la guia fonamental a la guia de dos modes, a la longitud d'ona de $1550 \mathrm{~nm}$. Per a això s'estudia i demostra experimentalment diferents dissenys a fi de obtindré el disseny més robust enfront les toleràncies de fabricació aconseguint un rendiment òptim.

A més, I'ús de DCs sobre guies ridge és comunament utilitzat i ofereix millors prestacions que el basat en guies strip, per eixe motiu aquesta Tesi estudia i avalua l'ús de ADCs sobre guies ridge per mitjà del mètode d'anàlisi dels índexs efectius dels supermodes parell i imparell. 
D'aquesta manera es realitza una comparació entre els dissenys òptims de les dos estructures (strip i ridge) amb l'objectiu d'esbrinar quin disseny ofereix millors prestacions.

Finalment, es proposa i estudia el disseny d'un acoblador grating capaç de multiplexar i demultiplexar els modes òptics del mode fonamental i del primer orde superior des de la guia òptica a la fibra òptica i viceversa. Per a això es proposen diferents dissenys amb l'objectiu d'aconseguir un disseny més tolerant i eficient enfront dels errors per desalineament obtenint un acoblament òptim.

L'ús de totes estes tècniques permetrà realitzar la multiplexació modal sobre els diferents escenaris i així augmentar l'ample de banda del sistema. 


\section{INDEX}

CHAPTER 1. INTRODUCTION ................................................... 1

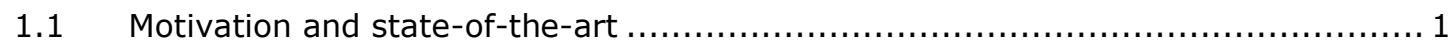

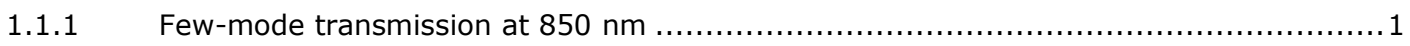

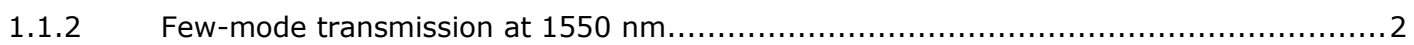

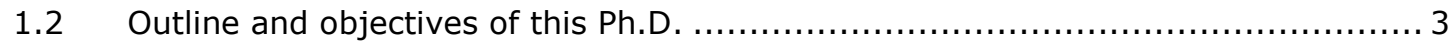

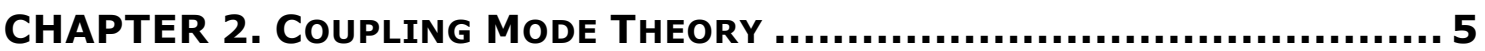

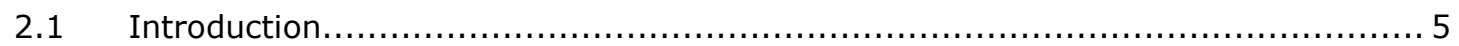

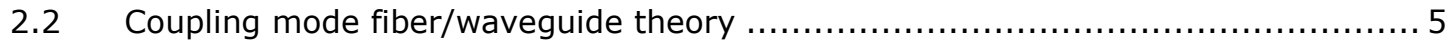

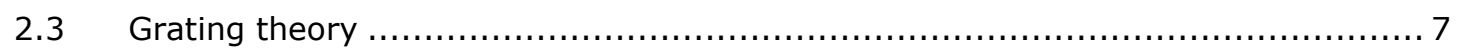

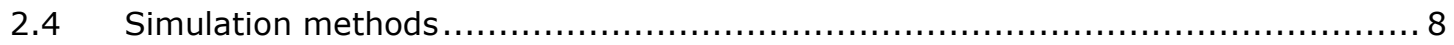

ChAPTER 3. Mode Division MultipleXing at 850 nM .................... 11

3.1 Mode-Selective Couplers for Two-Mode Transmission at $850 \mathrm{~nm}$ in Standard SMF 11

3.2 Mode Conversion for Mode Division Multiplexing at $850 \mathrm{~nm}$ in Standard SMF ...... 19

3.3 Modal Selectivity at $850 \mathrm{~nm}$ employing Standard Single-Mode Couplers: Theory and

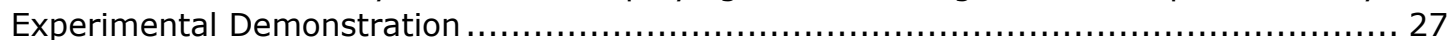

ChAPTER 4. Mode Division MultipleXing at 1550 nM ................... 35

4.1 Dimensional variation tolerant mode converter/multiplexer fabricated in SOI

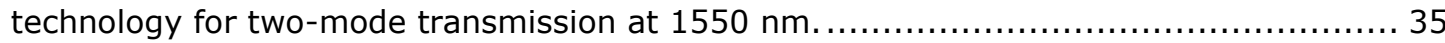

4.2 Design of Asymmetrical Directional Couplers on Ridge and Strip SOI technology with high dimensional variation tolerance

4.3 Bimodal grating coupler design on SOI technology for mode division multiplexing at $1550 \mathrm{~nm}$

CHAPTER 5. GENERAL DISCUSSION .........................................6 63

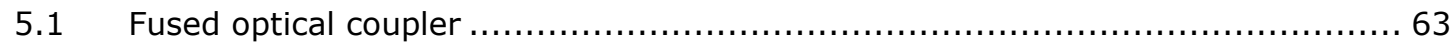

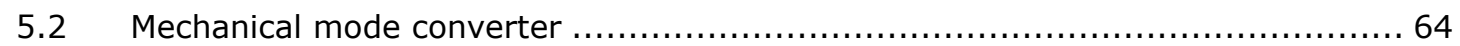

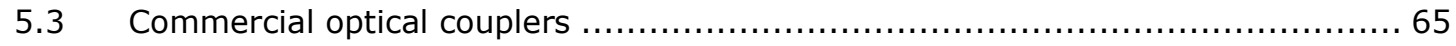

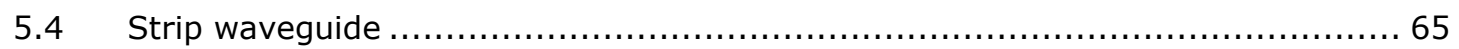

5.5 Supermodes based on strip and ridge waveguides $\ldots \ldots \ldots \ldots \ldots \ldots \ldots \ldots \ldots \ldots \ldots \ldots 6,6 \ldots \ldots \ldots \ldots \ldots$

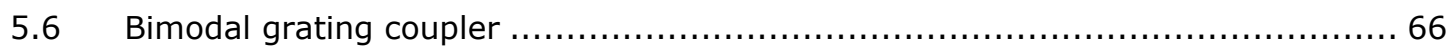

CHAPTER 6. CONCLUSION AND FURTHER WORK ............................. 69

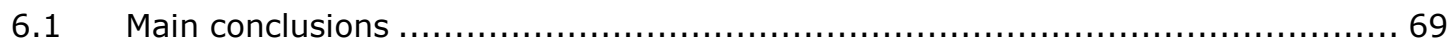

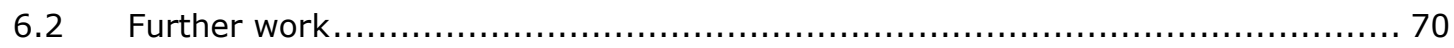

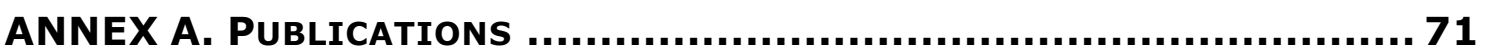

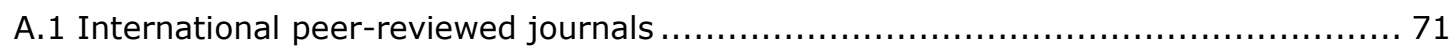

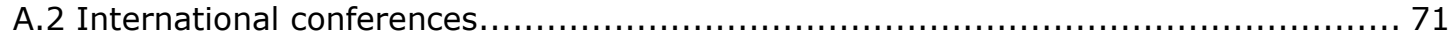

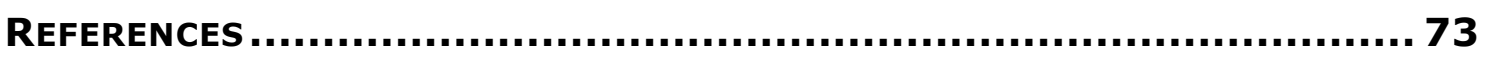





\section{CHAPTER 1. INTRODUCTION}

\subsection{Motivation and state-of-the-art}

In this Ph.D. thesis, different scenarios for mode division multiplexing (MDM) optical transmission are proposed, analyzed and investigated experimentally with the aim of increasing the single-user and overall transmission capacity (bitrate $\times$ number of users) in optical links, thus developing a further optical communications technology with direct application in next-generation optical networks.

MDM appears as a solution to overcome the capacity limit of the optical fiber [ESS10]. Due to the continuously growing internet traffic demand in the next decade, there is a need to increase the optical fiber capacity to accommodate a huge amount of traffic (machine-to-machine and video) [RIC13], [ESS10]. Different solutions to surpass the capacity limit as multi-core fibers (MCF) [MIZ16], multi-mode fiber (MMF) [RYF12] and multi-core multi-mode fibers [SAS17] are intensively investigated. However, the MCFs requires to incorporate multiple separate cores with sufficiently low crosstalk between neighboring cores. Also, the MMFs use a spatial filtering [MOR11], [FRA10] in order to limit the number of modes due to the high level of modal dispersion. However, few-mode fibers (FMFs) are considered as a complementary solution with the possibility to guide just a limited number of modes in MDM transmission.

Recently, the use of FMFs for MDM systems [BER82] has received considerable attention. Different solutions have been proposed, such as the possibility to reuse existing SMF infrastructures in the visible spectrum. This opens up the possibility of taking profit of the low-cost devices available in the market such as $10 \mathrm{~Gb} / \mathrm{s}$ VCSEL at $850 \mathrm{~nm}$ [TAT15]. On the other hand, it is possible to use a special fiber with a two-mode behavior at $1550 \mathrm{~nm}$ [KAS13]. Silicon on insulator (SOI) or planar lightwave circuit (PLC) processing technologies are optimal candidates to produce cost-effective optical devices for modal multiplexing in MDM transmission systems using TMF [HAN15], [WAN14].

The implementation of the aforementioned technologies allows an increase of the overall system capacity, providing enough bitrate for the multiple services such as the continuous increase of video consumption in bitrate-demanding formats (as for example the 4K [CIS17]).

\subsubsection{Few-mode transmission at $850 \mathrm{~nm}$}

Few-mode propagation has recently emerged as a promising approach to increase the optical transmission capacity. However, until the present moment, this technique has been implemented only for a specific photonic crystal fiber, which presents the disadvantage of a high cost and low availability [SAK11]. Consequently, the use of this type of fiber limits the development of optical transmission systems based on few-mode fibers.

Implementation of few-mode propagation in standard single-mode fibre (SSMF) requires employing wavelengths in the range of $850 \mathrm{~nm}$. In this way we can take advantage of the SSMF already deployed on the field, altogether readily the availability of optical sources such as VCSEL or LED, as well as low-cost photodetectors [VIS11], [KRU11]. Besides, it offers the possibility to reuse the already existing network infrastructure based on SSMF, just adding MDM transceivers to the networking equipment.

Although it has been paid low attention to transmission in optical fibers at $850 \mathrm{~nm}$ wavelength due to the high propagation losses (in contrast with the $\mathrm{C}+\mathrm{L}$ band), those are considerably low compared with polymer optical fibres (POF), as shown in Figure 1.1.1. 


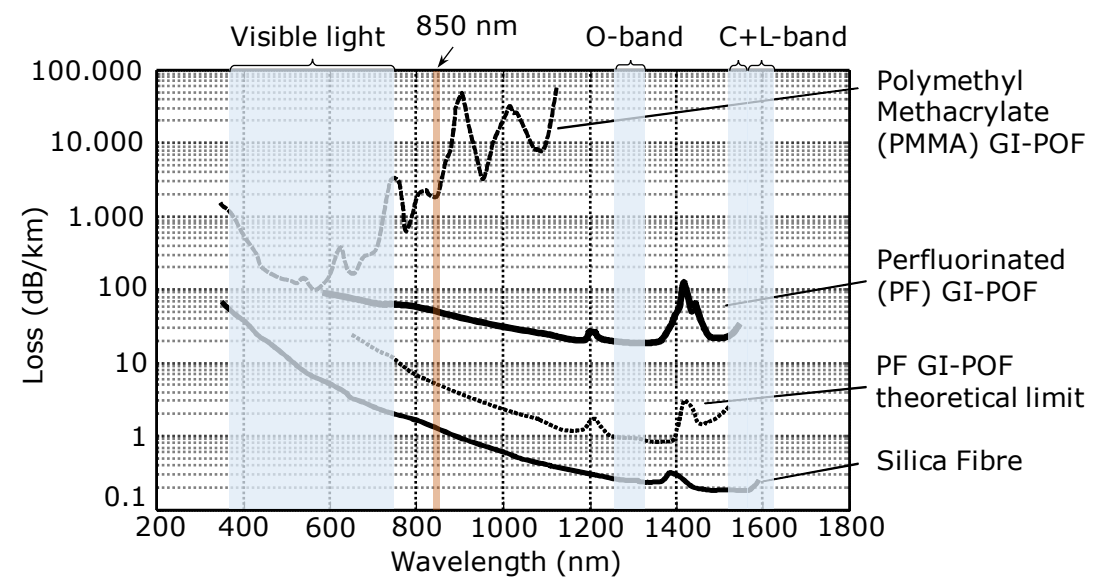

Figure 1.1.1. Propagation losses for different types of fibre [LLO14].

In the state-of-the-art, different optical transmissions over SSMF using visible light have been reported [HAN11], [TIA12]. Also, the use of free-space transmission is usually considered on the MDM experiments [MIL16].

Using the $850 \mathrm{~nm}$ wavelength opens up the possibility to multiplex two linear polarization (LP) modes when SSMF is chosen as the transmission media. The number of propagated modes is determined by the conventional definition of the normalized frequency $(V)$ :

$$
V=\frac{2 \pi a}{\lambda} \sqrt{n_{\text {core }}^{2}-n_{\text {cladding }}^{2}}
$$

Where $a$ is the core radius of the optical fiber, $\lambda$ is the wavelength and $n_{\text {core }}$ and $n_{\text {cladding }}$ are the refractive indexes of core and cladding, respectively.

In fact, the refractive indexes for the SMF- 28 at $850 \mathrm{~nm}$ are $n_{\text {core }}=1.4575$ and $n_{\text {cladding }}=1.4525$, obtaining a normalized frequency $V=3.74$. Figure 1.1 .2 represents the normalized propagation constant $(b)$ in relation with the normalized frequency $(V)$, i.e., the number of propagated modes depending on the $V$-cut value. From this figure, it can be stated that only the $\mathrm{LP}_{01}$ and $\mathrm{LP}_{11}$ modes are propagated (highlighted zone).

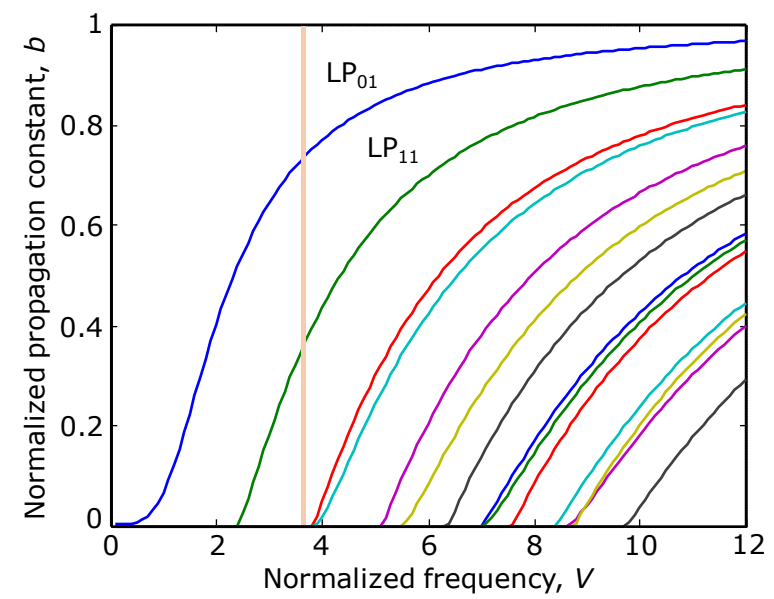

Figure 1.1.2. $b-V$ diagram for the SMF-28 fiber with a $V$-cut=3.74 propagating the $L P_{01}$ and $L P_{11}$ modes.

\subsubsection{Few-mode transmission at $1550 \mathrm{~nm}$}

Several MDM transmission systems using FMFs in the infrared band have been proposed [KAS13], [RYF12], [LI11]. Low-cost reliable mode converters and (de)multiplexers based on SOI or PLC processing technologies are the key devices on the success of MDM. Typically, the main techniques to convert/couple the modes using (de)multiplexers are the asymmetric directional couplers (ADCs) [DAI13], Y-junctions [SUN16], multimode interference [ZAN16], tapered directional couplers [ZHA15] or adiabatic couplers [XIN13]. 
The techniques based on PLC technology are the most commonly employed, offering low insertion loss, high yield rate, and mass productivity [DIN13], [HAN14]. However, higher sizes and higher bending radii are required. Instead, SOI technology offers the counterpart of the PLC devices with a reduced size and shorter bending radii [WAN14], [DAI13].

The optical fiber employed along the MDM transmission is a specialty fiber that acts as two-mode fiber (TMF) at $1550 \mathrm{~nm}$. For example, the SM2000 step-index fiber from Thorlabs $\left(11 \mu \mathrm{m}\right.$ core diameter with a $\mathrm{SiO}_{2}$ cladding, $n_{\text {core }}=1.4495$ at $1550 \mathrm{~nm}$ ) has a TMF behaviour at $1550 \mathrm{~nm}$. Thus, the SM2000 fiber obtained a $V=2.8093$ propagating the $L P_{01}$ and $L P_{11}$ modes (highlighted zone) as is depicted in Figure 1.1.3.

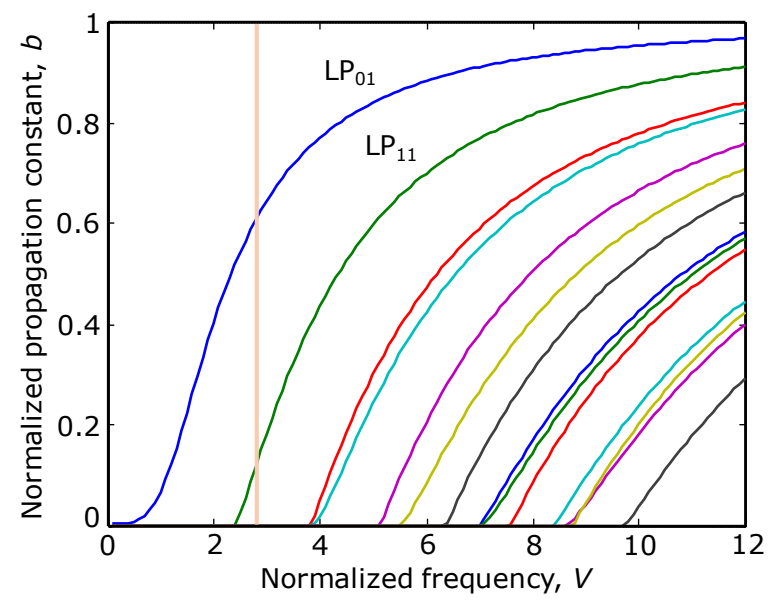

Figure 1.1.3. $b$-V diagram for SM2000 fiber with a $V$-cut $=2.8093$ propagating the $L P_{01}$ and $L P_{11}$ modes.

\subsection{Outline and objectives of this Ph.D.}

This Ph.D. thesis has been carried out in the Optical Access and Next-Generation Networks of the Nanophotonics Technology Center (NTC) at the Universitat Politècnica de València (UPV). The thesis was coordinated by Dr. Roberto Llorente (supervisor of the area in the NTC) and Dr. Juan Luis Corral from the Universitat Politècnica de València.

The research activities in this Ph.D. thesis target to increase the capacity of the optical links performing MDM in two different scenarios. On the one hand, the use of the $850 \mathrm{~nm}$ band takes profit of low cost reliable devices, such as VCSELs, and the possibility to reuse the current deployment of the SSMF. On the other hand, the use of a special fiber at $1550 \mathrm{~nm}$ wavelength, allows to use all the currently available equipment at the $C$ band and to design integrated circuits based on SOI technology to obtain low cost mode converters and (de)multiplexers. As a result of this study, more rigorous and suitable designs of the mode converters and multiplexers in the MDM systems will be provided. In particular, six different topics are considered throughout this work. In the first one, it is analyzed and simulated the design of a low-loss fused fiber mode-selective coupler for two-mode fiber transmission in the $850 \mathrm{~nm}$ band. Firstly, an asymmetrical coupler with different widths is assumed. Secondly, a symmetrical coupler with similar widths is considered. In both cases, the mode coupling is based on the phase mismatch condition. The second topic discussed is the analysis, design and fabrication of a mechanical mode converter over SSMF at $850 \mathrm{~nm}$. The main point is the tunability to adapt the mode converter to the tolerances in fiber specifications or laser performance. With this purpose, the design can be tuned in a $124 \mathrm{~nm}$ bandwidth offering a great operation bandwidth. Additionally, the transmission performance over a $1 \mathrm{~km}$ employing OOK modulation is experimentally evaluated. The third thematic area of this Ph.D. thesis is focused on the use of commercially available standard single mode couplers designed for the $1550 \mathrm{~nm}$ window (a low cost device) as mode multiplexer/demultiplexer of $L \mathrm{P}_{01}$ and $L \mathrm{LP}_{11}$ modes in a SSMF few mode link operating at $850 \mathrm{~nm}$. Both simulation and experimental results are presented. The fourth and fifth topic deal with asymmetrical directional couplers (ADCs) based on SOI technology. Firstly, it is analyzed, designed and fabricated an optimum mode converter 
and mode multiplexing/demultiplexing using a strip structure. Secondly, both structures (strip and ridge) are compared with the analysis of the supermodes in order to find an optimum design fabrication tolerances. In both cases, the designs were targeted to reduce ADC device fabrication requirements. Finally, the sixth and last topic is focused on the grating coupler design for simultaneously coupling both $\mathrm{LP}_{01}$ and $\mathrm{LP}_{11}$ modes using a TMF at $1550 \mathrm{~nm}$. Previously, the optimum design of the fourth topic was employed in order to convert the $\mathrm{TE}_{0}$ mode into $\mathrm{TE}_{1}$ mode. In this work, the coupling of the $L \mathrm{P}_{01}$ and $L \mathrm{P}_{11}$ modes is exhaustively analyzed and experimentally evaluated.

With regard to the Ph.D. thesis structure, it is organized as follows:

The herein first Chapter is devoted to the introduction. Next, Chapter 2 presents some fiber/waveguide basic concepts, such as the mode coupling theory, grating basic features, and simulation methods. The following two Chapters ( 3 and 4) contain a list of the most relevant publications from this Ph.D. thesis. There are a total of five published articles and one submitted for publication. The first three articles deal with the topic of MDM at $850 \mathrm{~nm}$ using SMF-28 with the design and simulation of symmetrical and asymmetrical optical couplers, the design and fabrication of a mechanical mode converter, and the use of commercially-available optical couplers designed for the $1550 \mathrm{~nm}$ window operating at $850 \mathrm{~nm}$. The remaining three articles are devoted to MDM at $1550 \mathrm{~nm}$ with the design, simulation and fabrication of an optimum ADC based on strip structure, the analysis and simulation of the ADCs based on strip and ridge structures, and the design, simulation and fabrication of an optimum grating coupler for both $\mathrm{LP}_{01}$ and $\mathrm{LP}_{11}$ modes. Chapter 5 contains a general discussion about the main findings of the work presented in Chapters 3 and 4. This Chapter is splitted into six subsections, each of them linked to its correspondent thematic area as described above in this introductory chapter. Finally, Chapter 6 summarises the most relevant conclusions from this work. 


\section{CHAPTeR 2. Coupling Mode Theory}

\subsection{Introduction}

The coupled-mode theory determines the interaction between the optical modes propagating through two different parallel waveguides/fibers that are spatially close. In section 2.2 , we consider the general formulation and solutions for the relevant case of two-mode coupling. It is based on the use of the mode coupling in a directional coupler. This structure is composed by two parallel waveguides or fibers separately a distance, $S$, and a coupling length, $L$, as it is shown in Figure 2.2.1. In section 2.3, we consider the basic formulation for periodic structures composed by several waveguide and optical fiber sections, the basic formulation of the periodic structures in the waveguide and optical fiber cases. Finally, in section 2.4 it is discussed the package software employed for the simulations of this thesis.

\subsection{Coupling mode fiber/waveguide theory}

The mode coupling theory is concerned with coupling spatial modes of differing polarizations, distributions, or both. The operation principle is based on the use of two (or more) arbitrarily shaped, but otherwise uniformly parallel waveguides placed in close proximity, as can be seen in Figure 2.2.1

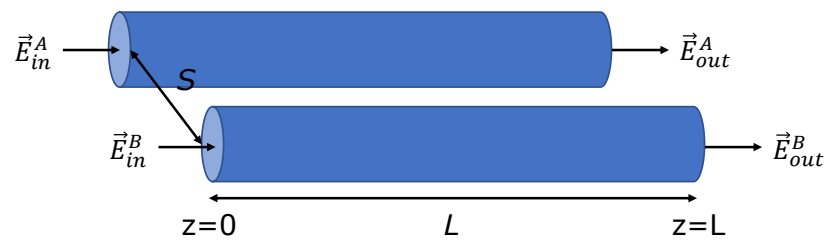

Figure 2.2.1. Mode coupling between two parallel optical fibers (or waveguides) for a separation $S$, and coupling length, $L$.

The optical fields in each waveguide, propagating along the same direction ( $z$ axis) can be expressed as

$$
\begin{aligned}
& \vec{E}^{A}(x, y, z, t)=A(z) \vec{\varepsilon}_{m n}^{A}(x, y) e^{j\left(\beta_{m n}^{A} z-\omega t\right)} \\
& \vec{E}^{B}(x, y, z, t)=B(z) \vec{\varepsilon}_{p q}^{B}(x, y) e^{j\left(\beta_{p q}^{B} z-\omega t\right)}
\end{aligned}
$$

where $\vec{\varepsilon}_{m n}^{A}(x, y)$ and $\vec{\varepsilon}_{p q}^{B}(x, y)$ are the modal profiles of the modes under consideration in each waveguide ( $m n$ in the waveguide $\mathrm{A}$ and $p q$ in the waveguide $\mathrm{B}$ ), while $\beta_{m n}^{A}$ and $\beta_{p q}^{A}$ are the propagation constants for both modes, respectively. The modal profiles and propagation constants correspond to the isolated waveguide modes, whereas the influence of the local modal profiles will be considered in the variation of the complex envelopes, $A(z)$ and $B(z)$, along the direction of propagation.

The complex envelopes at the output $(z=L)$ for both modes according to their values in the input $(z=0)$ can be expressed as [LIU07]

$$
\left[\begin{array}{c}
A(L) \\
B(L)
\end{array}\right]=\left[\begin{array}{cc}
\frac{\beta_{c} \cos \left(\beta_{c} L\right)-j \delta \sin \left(\beta_{c} L\right)}{\beta_{c}} e^{j \delta L} e^{j \kappa_{a a} L} & \frac{j \kappa_{a b}}{\beta_{c}} \sin \left(\beta_{c} L\right) e^{j \delta L} e^{j \kappa_{a b} L} \\
\frac{j \kappa_{b a}}{\beta_{c}} \sin \left(\beta_{c} L\right) e^{-j \delta L} e^{j \kappa_{b b} L} & \frac{\beta_{c} \cos \left(\beta_{c} L\right)+j \delta \sin \left(\beta_{c} L\right)}{\beta_{c}} e^{-j \delta L} e^{j \kappa_{b b} L}
\end{array}\right]\left[\begin{array}{c}
A(0) \\
B(0)
\end{array}\right]
$$

where $\kappa_{a} a$ and $\kappa_{b b}$ are the self-coupling coefficients that take into account the variation in the propagation constant of each mode due to the perturbation caused by the other waveguide; $\kappa_{a b}$ and $\kappa_{b a}$ are the mutual coupling coefficients of the waveguide B to A and vice versa which depend on the fiber separation, $S$; and $\delta$ is the phase mismatch. 
The phase mismatch has an important role in the coupling efficiency of the directional coupler and can be expresses as

$$
\delta=\frac{\left(\beta_{p q}^{B}+\kappa_{b b}\right)-\left(\beta_{m n}^{A}+\kappa_{a a}\right)}{2}
$$

Assuming $L$ is the length of the directional coupler, if only one mode is present at the waveguide $A$ input of the coupler $(B(0)=0)$, the optical power in both outputs (direct $(A)$ and coupled (B) paths) as a function of the input power $\mathrm{P}_{\mathrm{a}}(0)$ is

$$
\begin{gathered}
\frac{P_{a}(L)}{P_{a}(0)}=\left|\frac{A(L)}{A(0)}\right|^{2}=\left(\frac{\kappa_{a b} \kappa_{b a}}{\beta_{c}^{2}}\right) \cos ^{2}\left(\beta_{c} L\right)+\left(\frac{\delta}{\beta_{c}}\right)^{2} \\
\frac{P_{b}(L)}{P_{a}(0)}=\left|\frac{B(L)}{A(0)}\right|^{2}=\frac{\left|\kappa_{b a}\right|^{2}}{\beta_{c}^{2}} \sin ^{2}\left(\beta_{c} L\right) \\
\beta_{c}=\sqrt{\kappa_{a b} \kappa_{b a}+\delta^{2}}
\end{gathered}
$$

Finally, the coupling efficiency is defined as

$$
\eta=\frac{P_{b}(L)}{P_{a}(0)}=\frac{\left|\kappa_{b a}\right|^{2}}{\beta_{c}^{2}} \sin ^{2}\left(\beta_{c} L\right)
$$

Therefore, the optical power is periodically exchanged between both waveguides appearing the first maximum in the coupled output for a distance:

$$
L_{c}=\frac{\pi}{2 \beta_{c}}
$$

The power transfer will be maximized when the phase-matching condition is fulfilled $(\delta=0)$, which implies that the propagation constants of both modes in the coupler have to be the same and, therefore, the refractive indices will also be equal.

The effective refractive indexes of the optical modes involved play an important role in order to fulfill the phase-mismatch condition $(\delta=0)$. When the coupler is made of similar waveguides (DC), the phase match condition is always fulfilled if the modes to be coupled are the same one $(A=B)$, regardless of the wavelength. However, in an ADC, the effective indices of the modes to be coupled, which are wavelength dependent, must be matched by properly selecting the dimensions of the respective waveguides to be used.

Considering an $A D C$ at $850 \mathrm{~nm}$, the effective refractive index of $\mathrm{LP}_{01}$ in SMF-28 must match the $\mathrm{LP}_{11}$ index in TMF which can be accomplished by reducing the core radius of a SMF- 28 fiber to $2.05 \mu \mathrm{m}$, as can be seen in Figure 2.2.2.

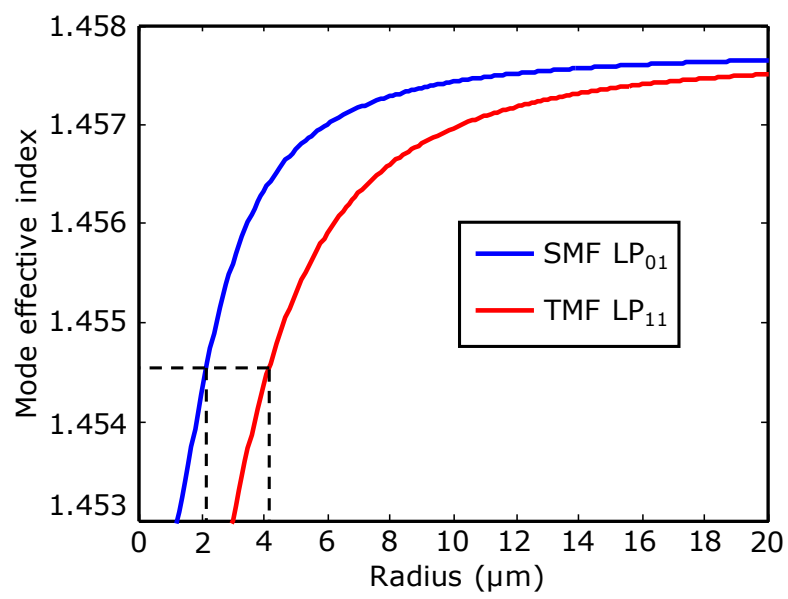

Figure 2.2.2. The phase matching graphs for the $L P_{01}$ in the SMF and the $L P_{11}$ in the TMF. 
Likewise, the optical waveguides in the $1550 \mathrm{~nm}$ operation band have a similar behavior. In this case, the $\mathrm{TE}_{0}$ mode is propagated over the single-mode waveguide $(0.45 \mu \mathrm{m}$ width) and the two-mode waveguide is increased from $0.45 \mu \mathrm{m}$ to $0.962 \mu \mathrm{m}$ in order to fulfill the phase mismatch between the $\mathrm{TE}_{0}$ and $\mathrm{TE}_{1}$ modes, achieving the same effective index, as it is shown in Figure 2.2.3.

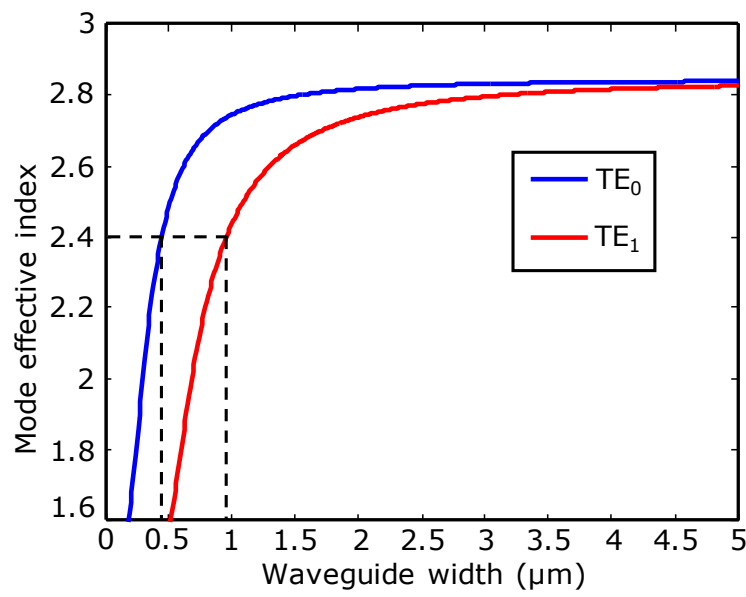

Figure 2.2.3. The phase matching graphs for the $T E_{0}$ in the single-mode waveguide and the $T E_{1}$ in the two-mode waveguide.

\subsection{Grating theory}

An asymmetric perturbation can be created in a periodic structure by means of a mechanical grating in the optical fiber applying pressure or using a grating coupler based on silicon-oninsulator (SOI) technology, as it is depicted in Figure 2.3.1. Both techniques are useful to generate a mode conversion or mode multiplexing.

(a)

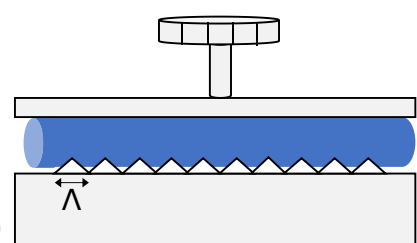

(b)

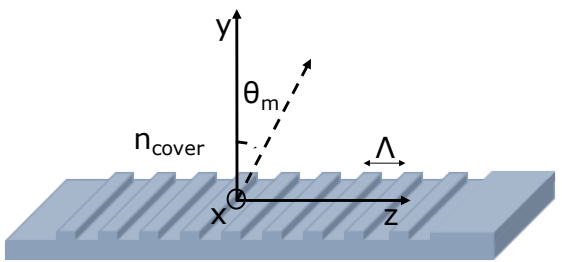

Figure 2.3.1. Periodic structure for: (a) optical fiber with applied pressure and (b) grating coupler.

The mechanical grating is a periodic structure defined by a grating period $(\Lambda)$ with $N$ coupling points. The structure apply pressure over the fiber, generating an asymmetric perturbation inside the optical fiber. The propagated $L P_{01}$ mode is converted into $L P_{11}$ mode in function of the grating period that defines the wavelength where the efficiency to convert the $L P_{01}$ to $L P_{11}$ is maximum. The conversion efficiency is expressed as [VEN96]

$$
\begin{gathered}
\frac{P_{11}}{P_{01}}=\frac{\sin ^{2}\left(\kappa \cdot L \sqrt{1+\left(\frac{\delta}{\kappa}\right)^{2}}\right)}{1+\left(\frac{\delta}{\kappa}\right)^{2}} \\
\delta=\frac{1}{2}\left\{\frac{2 \pi}{\lambda}\left(n_{L P 01}-n_{L P 11}\right)-\frac{2 \pi}{\Lambda}\right\}
\end{gathered}
$$

where $P_{01}$ is the power of the $L P_{01}$ mode, $P_{11}$ is the power of the $L P_{11}$ mode, $k$ is the coupling coefficient, $L$ is the grating length, $\delta$ is the detuning parameter, $\lambda$ is the wavelength, $n_{L P O 1}$ and $n_{L P 11}$ are the effective refractive indexes of the $L P_{01}$ and $L P_{11}$ modes and $\Lambda$ is the grating period. Thus, the conversion efficiency is maximized when $\delta=0$ and, therefore, the grating period can be defined as 


$$
\Lambda=\frac{\lambda}{n_{L P 01}-n_{L P 11}}
$$

Finally, in a period structure with equal pressure in each coupling point, the conversion efficiency is simplified to [GIL12]

$$
\frac{P_{11}}{P_{01}}=\sin ^{2}\left(N \cdot \sin ^{-1}(\kappa)\right)
$$

In the case of a grating coupler, the periodic structure is defined by the Bragg condition which describes the relation between the wave-vectors of the incident and diffracted waves. The equation can be defined as [GAL08]

$$
\frac{2 \pi}{\lambda} n_{\text {cover }} \sin \left(\theta_{\mathrm{m}}\right)=\frac{2 \pi}{\lambda} n_{\text {eff }}+m \frac{2 \pi}{\Lambda}
$$

where $\lambda$ is the design wavelength, $n_{\text {cover }}$ is the refractive index of the cover substrate, $\theta_{m}$ is the tilt angle, $n_{\text {eff }}$ the effective index of the optical mode in the grating waveguide, $\Lambda$ is the grating period and the integer $m$ is the diffraction order.

The coupling of the $T E_{0}-L_{01}$ and $T E_{1}-L_{11}$ modes from/to the grating coupler is always fulfilled if the waveguide width is sufficiently large in order to obtain similar effective refractive indexes. Thus, the coupling efficiency from fiber to waveguide is the same as from waveguide to fiber due to reciprocity theorem. When the electromagnetic fields at the position of the fiber facet are known, the coupling efficiency to fiber becomes [ROE06]

$$
\eta=\left|\iint_{S} E \times H_{\text {fib }}^{*} d S\right|^{2}
$$

where $E$ is the normalized electrical field of the diffracted wave from the grating, and $H_{\text {fib }}$ is the normalized magnetic fields of the optical mode in the fiber and $S$ is the fiber facet. Equation (2.2.15) is a good approximation, even if the grating is covered by air instead of silica [TAI05].

\subsection{Simulation methods}

In this Ph.D. thesis three different simulation methods implemented in the RSoft Component Design Suite were employed [RSOFT].

The first simulation package is known as BeamPROP and it is based on advanced finitedifference beam propagation (BPM) techniques. These techniques are employed to solve the well-known parabolic or paraxial approximation of the Helmholtz equation. The method employed by the simulation tool BeamPROP is the most widely used propagation technique for modelling integrated and fiber optic photonic devices, and most commercial software for such modelling is based on it. The reasons are the fast and accurate results in accordance with the number of grid points, which can be optimized when the number of grid points are lesser than $10 \mathrm{~nm}$. However, it is not suitable when the waveguide or optical fibers have a little bend in the design due to the paraxial approximation of the Helmholtz equation that only takes into account the propagation in the z-direction.

Second, the FullWAVE simulation package is based on the well-known finite-difference timedomain (FDTD) technique. This technique consists of a solution from Maxwell's equations without any approximation or restriction, allowing good results for the cases where the BPM method fails, namely high index contrast devices or blended components. Additionally, FDTD easily lends itself to cluster computing taking profit of several simultaneous computers in order to solve a complex problem. However, a full three-dimensional simulation would imply a long time computing as well as a huge of amount of RAM for the most common devices such as waveguide couplers, rings, etc. However, the BPM is the perfect package for the type of components analyzed in this Ph.D. thesis. 
Finally, FemSIM is the last simulation package which it is based on a Finite Element Method (FEM) technique to calculate modes, both waveguide and cavity, of any 1D or 2D cross-section. It is a full-vector implementation for both propagating and leaky waveguide modes. 


\title{
ChAPTER 3. Mode Division MultipleXing At 850 nM
}

\subsection{Mode-Selective Couplers for Two-Mode Transmission at $850 \mathrm{~nm}$ in Standard SMF}

\section{BIBLIOGRAPHY RECORD}

- Authors: Juan L. Corral, David Garcia-Rodriguez and Roberto Llorente

- Publication: IEEE Photonics Technology Letters, vol. 28, no. 4

- Year: 2016

- Pages: $425-428$

- Keywords: Few-mode fiber, mode converter, mode multiplexer, optical fiber coupler, space division multiplexing

\begin{abstract}
The optimal design of a low-loss fused fiber mode-selective coupler for two-mode fiber transmission in the $850 \mathrm{~nm}$ band is presented. The coupler is based on precise phase matching of the propagation constants in each arm of a weakly fused fiber coupler. The designed component permits both mode converter and mode multiplexer/demultiplexer operation, thus enabling modal multiplexing transmission in this band with no additional component. The presented design is evaluated by simulation considering two types of structures, leading to asymmetric and symmetric coupler configurations. Mode converter and mode multiplexer operation is achieved with $93.5 \%$ efficiency in the band of $845-855 \mathrm{~nm}$. Mode demultiplexer operation is achieved with an extinction ratio exceeding $20.4 \mathrm{~dB}$ in the same band.
\end{abstract}




\section{INTRODUCTION}

Recently, significant research efforts have been directed to increase the capacity of fiber optic communication networks by adding spatial division multiplexing or mode division multiplexing (MDM) to current technology [1]. Mode converters and mode multiplexers/demultiplexers are key components in a MDM system to combine and split the different modulated optical signals.

Mode couplers based on free space have been successfully used in MDM experiments [2] but the devices based on directional couplers (DC) offer a more compact and cheap solution, with less losses and with direct integration with optical fiber. Recently, the use of a fiber based directional coupler for a MDM system at $1550 \mathrm{~nm}$ has been theoretically [3] and experimentally $[4,5]$ considered. However, the proposed system is based on the use of a specialty fiber with a two-mode behaviour at $1550 \mathrm{~nm}$. Thus, the huge amount of standard single-mode fiber already deployed could not be used. Instead, standard single-mode fiber (SSMF) can be used as a two-mode fiber (TMF) if short wavelengths below cutoff wavelength are chosen.

For instance, a fiber directional coupler has been reported as a mode coupler at $1080 \mathrm{~nm}$ in a MDM link based on SSMF but an additional fiber grating was required to convert modes [6]. We propose to use the band around $850 \mathrm{~nm}$ where low cost VCSELs are available with modulation bandwidth beyond $10 \mathrm{~Gb} / \mathrm{s}$. This band is widely used for short range high data rate optical links with multimode fiber (MMF). The use of a fiber bundle composed of 4 or 10 parallel multimode fibers is already standardized as a way to multiply link capacity in the $850 \mathrm{~nm}$ band [7] but the use of MDM would be a more efficient way to increase the data rate capacity of a single fiber.

The use of SSMF at the $850 \mathrm{~nm}$ band has been proposed by including mode filters to avoid the higher order modes but without taking profit of the capacity increase available if MDM were used $[8,9]$. The availability of low cost devices to convert, multiplex and demultiplex spatial modes in the $850 \mathrm{~nm}$ band on SSMF could ease the development of this multiplexing technique.

In this letter, the use of directional couplers as mode selective couplers for SSMF at $850 \mathrm{~nm}$ is analysed and optimal designs in terms of coupling efficiencies and extinction ratios are obtained. The organization of this letter is as follows: in Section II the coupler model is described, Section III presents the optimal design of an asymmetric coupler working as mode converter and mode multiplexer/demultiplexer whereas Section IV is devoted to a symmetric coupler working as a mode multiplexer/demultiplexer. Finally, some remarks and conclusions are summarized in Section $\mathrm{V}$.

\section{MODEL}

The basic concept of the MDM link is depicted in Figure 3.1.1. It consists of two lasers emitting at a wavelength (e.g. $850 \mathrm{~nm}$ ) below the SSMF cutoff wavelength. In this configuration, the SSMF behaves effectively as a two-mode fiber (TMF). The optical signals coming from the lasers will correspond to the $\mathrm{LP}_{01}$ mode if the lasers are properly aligned to the fiber centers or if the pigtails are made of fiber operating as single mode at $850 \mathrm{~nm}$ [9]. A mode converter $\left(L P_{01}\right.$ to $\left.L P_{11}\right)$ and a mode multiplexer are needed at the transmitter in order to transfer the modulated optical carriers coming from both lasers to the $L P_{01}$ and $L P_{11}$ modes in the SSMF. At the receiver side, a mode demultiplexer will be needed to direct each mode to the corresponding photodiode; however, the mode conversion ( $L P_{11}$ in the SSMF to $L P_{01}$ impinging photodiode PD2) is not strictly needed if the fiber connecting the mode demultiplexer to the PD2 photodiode is also SSMF. 


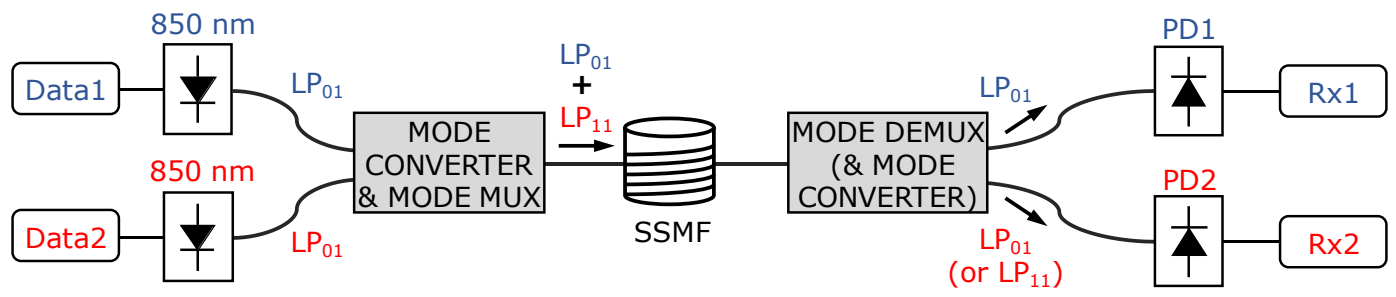

Figure 3.1.1. Scheme for mode-division multiplexing (MDM) at short wavelengths with SSMF as two-mode fiber (TMF) optical transmission media.

We propose the use of fiber based directional couplers as mode converter, mode multiplexer and mode demultiplexer in a MDM system, as depicted in Figure 3.1.1. The waveguide structure of a fiber based mode coupler is shown in Figure 3.1.2. This coupler is realized by weakly fusing two similar (symmetric coupler) or dissimilar (asymmetric coupler) fibers together. The principle of the asymmetric coupler is to phase match the fundamental mode in one fiber to a high order mode in a TMF in order to achieve a conversion to the high order mode. In a symmetric coupler, no mode conversion is obtained but the signal is coupled from one fiber to the other depending on the coupler geometry. The weak fused technique will ensure that the fiber geometries are kept and an accurate index matching is achieved.

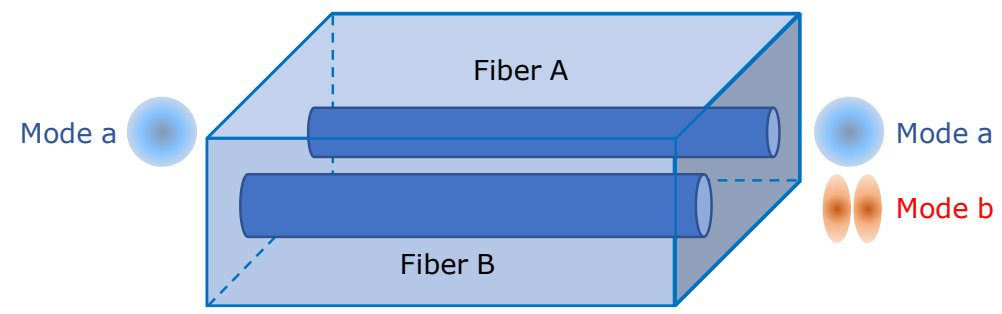

Figure 3.1.2. Waveguide structure of the fiber-based mode coupler.

According to Figure 3.1.2 the fiber coupler is made of two fibers (A, B) that are fused together with their cores surrounded by the cladding of both fibers and keeping a constant distance, $d$, between them. We are interested in studying the coupling between spatial mode $a$ in fiber $A$ (power $P_{A}$, propagation constant $\beta_{a}$ ) and spatial mode $b$ in fiber $B$ (power $P_{B}$, propagation constant $\beta_{b}$ ). According to the coupled-mode theory if the only optical signal present at the coupler input is mode $a$ at the input of fiber $A$, the optical power at both outputs (direct and coupled paths) of the coupler of length $L$ as a function of the input power, $P_{A}(0)$, can be expressed as [10]:

$$
\begin{gathered}
\frac{P_{A}(L)}{P_{A}(0)}=\left(\frac{\kappa_{a b} \kappa_{b a}}{\beta_{c}^{2}}\right) \cos ^{2}\left(\beta_{c} L\right)+\left(\frac{\delta}{\beta_{c}}\right)^{2} \\
\frac{P_{B}(L)}{P_{A}(0)}=\frac{\left|\kappa_{b a}\right|^{2}}{\beta_{c}^{2}} \sin ^{2}\left(\beta_{c} L\right) \\
\delta=\frac{\left(\beta_{b}+\kappa_{b b}\right)-\left(\beta_{a}+\kappa_{a a}\right)}{2} \\
\beta_{c}=\sqrt{\kappa_{a b} \kappa_{b a}+\delta^{2}}
\end{gathered}
$$

where $\kappa_{a a}, \kappa_{b b}, \kappa_{a b}$ and $\kappa_{b a}$ are the self-coupling and mutual coupling coefficients, respectively, and $\delta$ is the phase mismatch term which plays an important role in the coupling efficiency of the coupler. Finally, the coupling efficiency is defined as:

$$
\eta=\frac{P_{B}(L)}{P_{A}(0)}=\frac{\left|\kappa_{b a}\right|^{2}}{\beta_{c}^{2}} \sin ^{2}\left(\beta_{c} L\right)
$$

It is clear from (3.1.1) and (3.1.2) that maximum power coupling from fiber A to fiber B will require the phase mismatch, $\delta$, to be zero. In this case, the maximum coupling efficiency will 
be achieved when $\sin \left(\beta_{c} L\right)=1$. When the phase-match condition is fulfilled, $\beta_{c}$ depends on the mutual coupling coefficients which depend on the fiber separation, $d$, and the spatial modes profiles to be coupled.

When the coupler is made of similar fibers (symmetric coupler) the phase match condition is always fulfilled if the modes to be coupled are the same one $(a=b)$. However, in an asymmetric coupler the effective indexes of the modes to be coupled must be matched by properly selecting the features of the respective fibers to be used. The easiest way to adapt the fiber parameters is to pre-taper one of the fibers during the coupler fabrication in order to reduce its core diameter [4].

Once the effective indexes of the modes to be coupled are matched and the fibers spacing is fixed, the mutual couplings coefficients are known (they will be higher for closer fibers) and the maximum coupling efficiency will be obtained for different coupling lengths according to equation (3.1.5).

The fiber to be used for the couplers and the optical link will be standard single mode fiber; namely, SMF-28 from Corning Inc. $(8.2 \mu \mathrm{m}$ core diameter, $\Lambda=0.36 \%$ refractive index difference and $\mathrm{S} \mathrm{SiO}_{2}$ cladding). This fiber will behave as a two-mode fiber at $850 \mathrm{~nm}$ propagating the $\mathrm{LP}_{01}$ and $\mathrm{LP}_{11}$ (including odd and even degenerated versions) linear polarization modes.

A full vectorial Beam Propagation Method has been used to compute the modal effective index and to simulate the different mode selective couplers. The calculated refractive indexes for the SMF-28 fiber at $850 \mathrm{~nm}$ are $n_{\text {core }}=1.4577, n_{\text {cladding }}=1.4525, n_{L P 01}=1.4564$ and $n_{L P 11}=1.4545$. The relative difference between both modes indexes $(0.13 \%)$ will assure that no modal coupling will appear along the SSMF link as the measurements in [8] confirmed.

\section{ASYMMETRIC COUPLER}

The asymmetric coupler is made of a single mode fiber (SMF) propagating only the fundamental mode and a two-mode fiber propagating both $L P_{01}$ and $L P_{11}$ modes. In this case, the $L P_{01}$ mode entering the coupler through the SMF must be converted to $L P_{11}$ at the TMF output of the coupler. Thus, the effective index of $L P_{01}$ in SMF must match the $L P_{11}$ index in TMF which can be accomplished by reducing the core diameter of a SMF-28 fiber to $4.17 \mu \mathrm{m}$. This reduction of SMF-28 core diameter up to $51 \%$ of its original core diameter can be accomplished by pre-tapering the fiber during the fabrication of the coupler $[3,4]$.

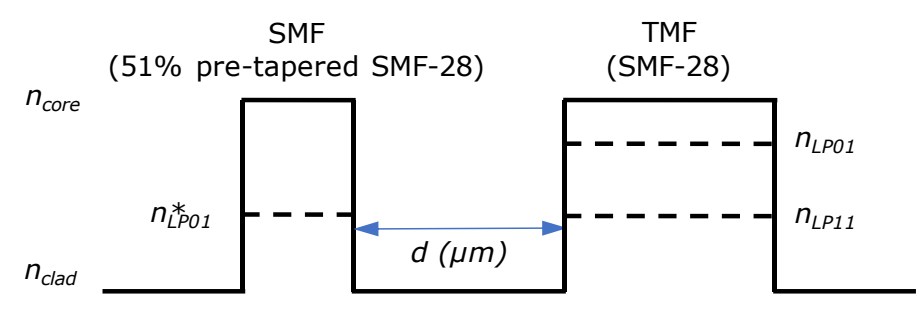

Figure 3.1.3. Refractive index profile of the asymmetric coupler.

Once the core diameter of the SMF-28 fiber is pre-tapered to $51 \%$ of the original core diameter, the fiber becomes single-mode at $850 \mathrm{~nm}$ and the effective index of the fundamental mode, $L P_{01}$, changes to $n *^{L P O 1}=1.4545$, the same index as the $L P_{11}$ mode in TMF as depicted in Figure 3.1.3. Now, the phase match condition is fulfilled and the maximum coupling efficiency from $L P_{01}$ in SMF to $L P_{11}$ in TMF can be obtained. This asymmetric coupler has been simulated at $850 \mathrm{~nm}$ for different values of fiber separation, $d(\mu \mathrm{m})$ and coupling length, $L(\mathrm{~mm})$ and the results are shown in Figure 3.1.4. As expected, for a fixed fiber separation, different coupling lengths achieve the maximum coupling efficiency according to the sinusoidal expression in equation (3.1.5). For wider fiber separations, the mutual coupling coefficients are reduced and the coupler requires longer lengths to achieve the maximum efficiency. 


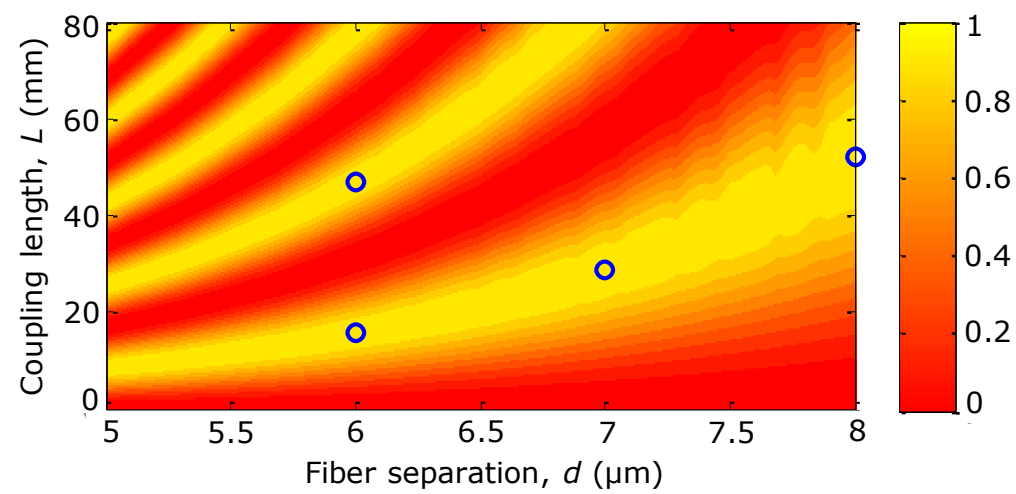

Figure 3.1.4. $L P_{01} \rightarrow L P_{11}$ coupling efficiency for an asymmetric mode-selective coupler with the refractive index profile from Figure 3.1.3.

Different combinations of fiber separation and coupler length offering the maximum coupling efficiency can be selected from Figure 3.1.4. In order to compare some of the combinations, the performance of the coupler has been assessed, both for the design wavelength of $850 \mathrm{~nm}$ and for different wavelengths around $850 \mathrm{~nm}$. The results are shown in Figure 3.1.5 for the four cases: $\left(d=6 \mu \mathrm{m}, L_{1}=15.66 \mathrm{~mm}\right),\left(d=6 \mu \mathrm{m}, L_{2}=46.95 \mathrm{~mm}\right),(d=7 \mu \mathrm{m}, L=28.56 \mathrm{~mm})$ and $(d=8 \mu \mathrm{m}, L=52.17 \mathrm{~mm})$ highlighted in Figure 3.1.4.
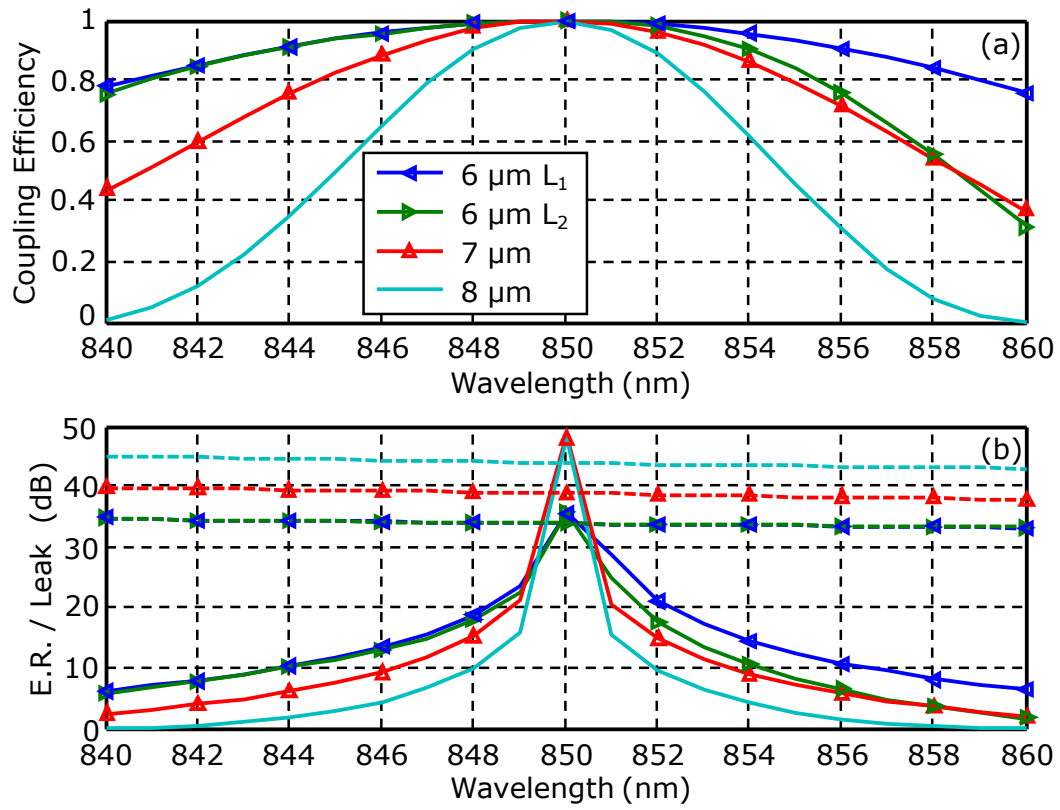

Figure 3.1.5. (a) $L P_{01} \rightarrow L P_{11}$ coupling efficiency, (b) $L P_{11}$ extinction ratio and $L P_{01}$ leakage (dashed line) for the asymmetric coupler from Figure 3.1.3 for different combinations of fiber separation, $d$, and coupler length, $L$.

When the asymmetric coupler is used as a mode converter and multiplexer as in the transmitter in Figure 3.1.1, the most important parameters are the coupling efficiency from the $L P_{01}$ input in the SMF to the $L P_{11}$ output in the TMF and the insertion losses for the $L P_{01}$ mode from input to output of the TMF. The insertion losses for the $L \mathrm{P}_{01}$ mode in the TMF are negligible as this mode is not coupled to the SMF and the length of the fiber is too short to introduce any significant loss. The coupling efficiency for this case is shown in Figure 3.1.5 where all four cases offer an efficiency better than 99\% (coupling losses below $0.05 \mathrm{~dB}$ ) at the design wavelength. However, the performance of the coupler for a broader bandwidth is quite dependent on the fiber spacing of the coupler and the best coupling efficiency is obtained for the lower spacing and the shorter coupling length $\left(d=6 \mu \mathrm{m}, L_{1}=15.66 \mathrm{~mm}\right)$ with a $93.5 \%$ (corresponding to a $0.3 \mathrm{~dB}$ insertion loss) coupling efficiency for a $10 \mathrm{~nm}$ bandwidth around $850 \mathrm{~nm}$. 
The asymmetric coupler could be used in the receiver side of the link as a mode converter and demultiplexer. In this case, both $\mathrm{LP}_{01}$ and $\mathrm{LP}_{11}$ modes enter the coupler through the TMF input and they are split into the TMF $\left(\mathrm{LP}_{01}\right)$ and the SMF $\left(\mathrm{LP}_{11}\right)$ outputs. The most important parameters to check besides the ones already considered in the multiplexer will be the extinction ratio of the $L P_{11}$ mode at the TMF output and the undesired leakage of the $L P_{01}$ mode from the TMF to the SMF. These parameters are plotted in Figure 3.1.5 where values above $34 \mathrm{~dB}$ are observed at $850 \mathrm{~nm}$. However, when a $10 \mathrm{~nm}$ bandwidth is considered the $\mathrm{LP}_{11}$ extinction ratio is quite reduced with the best performance obtained again for the coupler with the lower spacing and the shorter coupling length with an extinction ratio above $11.6 \mathrm{~dB}$ and a leakage above $33.6 \mathrm{~dB}$.

The asymmetric coupler shows an outstanding performance as mode converter and multiplexer but its performance as mode demultiplexer would be limited by the sensitivity of the $\mathrm{LP}_{11}$ extinction ratio to deviations of the laser wavelength. This limitation in its use as mode demultiplexer can be easily avoided by adding a modal filter after the TMF output of the coupler. The easiest implementation of this modal filter is to add some thigh turns to the fiber coming out of the TMF output of the coupler as more than $20 \mathrm{~dB}$ attenuation has been reported for 30 turns around a cylinder of 9-mm radius [8].

Concerning the demultiplexer, it is important to point out that, as it happens with all mode-selective couplers, the $\mathrm{LP}_{11}$ mode entering the coupler must be properly aligned (axial orientation of the mode aligned with the plane containing the fibers of the coupler, horizontal axis in Figure 3.1.2) which can be easily accomplished by adding an inline polarization controller acting as a mode rotator before the demultiplexer [9].

\section{SYMMETRIC COUPLER}

Compared with an asymmetric coupler, the propagation constants (thus, the effective refractive indices) of the same modes in the two fibers of a symmetric coupler are always matched at any given wavelength, and thus the device can be broadband. On the other hand, additional mode conversion, like a long-period fiber Bragg grating (LPFBG), is needed before mode coupling if the symmetric coupler is to be used as multiplexer in the transmitter in Figure 3.1.1 [5,6].

However, the symmetric coupler can be advantageously used as a mode demultiplexer at the receiver side in the setup shown in Figure 3.1.1 without any additional mode conversion as the $L P_{01}$ and $L P_{11}$ spatial modes coming from the fiber link will be split, and each mode $L P_{01}$ or $L P_{11}$ will impinge the corresponding photodiode without any mode conversion.

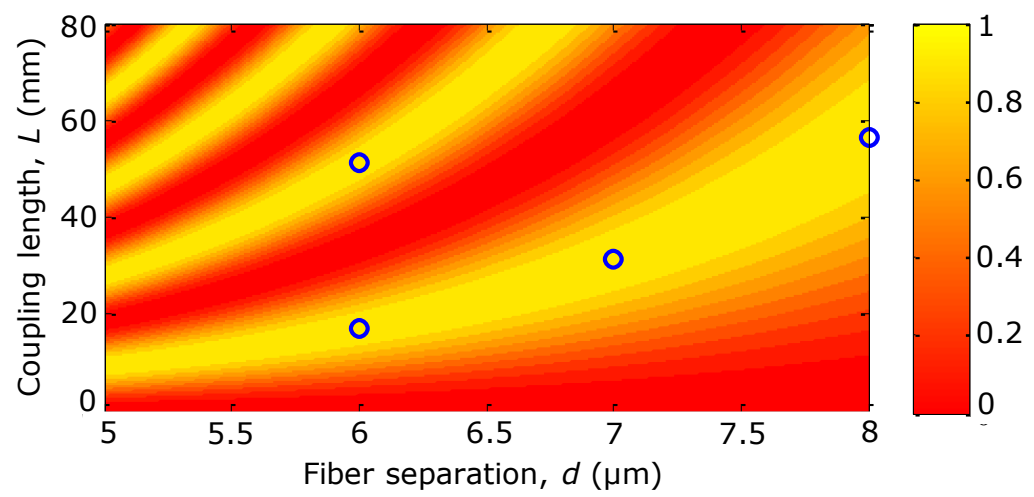

Figure 3.1.6. $L P_{11}$ coupling efficiency for a symmetric mode-selective coupler made of SMF-28 fiber.

Figure 3.1.6 show the $L P_{11}$ coupling efficiency of the symmetric coupler at $850 \mathrm{~nm}$ for different values of fiber separation, $d(\mu \mathrm{m})$ and coupling length, $L(\mathrm{~mm})$. Figure 3.1.7 show the simulation results of the four cases: $(d=6 \mu \mathrm{m}, L=17.01 \mathrm{~mm}),(d=6 \mu \mathrm{m}, L=51.09 \mathrm{~mm}),(d=7 \mu \mathrm{m}$, $L=31.14 \mathrm{~mm})$ and $(d=8 \mu \mathrm{m}, L=56.58 \mathrm{~mm})$ highlighted in Figure 3.1 .6 corresponding to different designs offering the maximum coupling efficient at $850 \mathrm{~nm}$. 

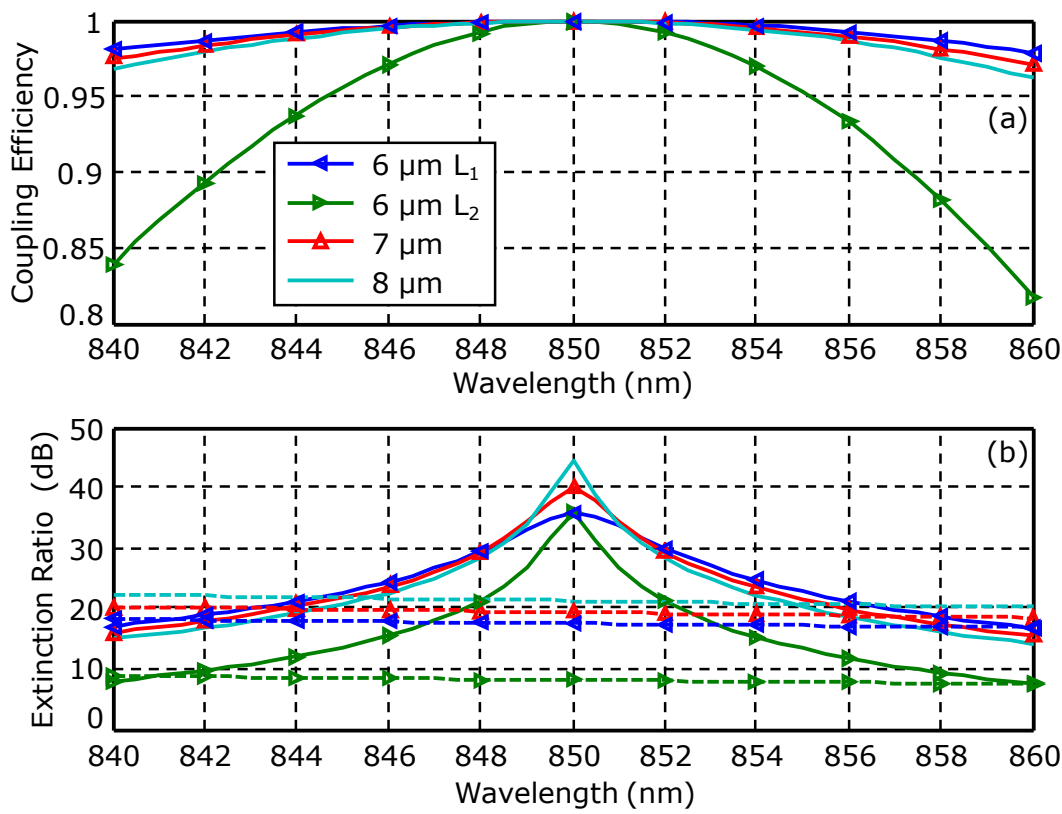

Figure 3.1.7. (a) $L P_{11}$ coupling efficiency, (b) $L P_{11}$ and $L P_{01}$ (dashed line) extinction ratios for the symmetric coupler for different combinations of fiber separation, $d$, and coupler length, $L$.

As expected the $\mathrm{LP}_{11}$ coupling efficiency of the symmetric coupler is quite less sensitive to wavelength variation and a coupling efficiency better than $99 \%$ (insertion loss below $0.04 \mathrm{~dB}$ ) can be achieved in a $10 \mathrm{~nm}$ bandwidth around $850 \mathrm{~nm}$ when the minimum coupling length for any fixed fiber separation is selected. The insertion loss for the $\mathrm{LP}_{01}$ (straight path) in these three cases is better than $0.09 \mathrm{~dB}$ in the same bandwidth. Thus, the use of the symmetric coupler as a mode multiplexer in Figure 3.1.1 could be an interesting option if an external mode converter could be easily obtained. In other case, the asymmetric coupler from section III would be a better option.

In order to consider the use of the symmetric coupler as a demultiplexer in the receiver side of Figure 3.1.1, the extinction ratio for both modes in the opposite output of the coupler should be considered. In the symmetric coupler, both modes, $\mathrm{LP}_{01}$ and $L \mathrm{P}_{11}$, are coupled, although the $L P_{01}$ mode requires quite longer coupling lengths. If the $L P_{01}$ extinction ratio for both couplers with $d=6 \mu \mathrm{m}$ are compared $\left(17.5 \mathrm{~dB}\right.$ for $L_{1}$ and $8.2 \mathrm{~dB}$ for $L_{2}$ at $850 \mathrm{~nm}$ ) it is clear that the minimum coupling length must be selected to minimize the undesired coupling of the $\mathrm{LP}_{01}$ mode. The corresponding insertion losses for the $\mathrm{LP}_{01}$ mode in the straight path are also better for shorter couplers ( $0.08 \mathrm{~dB}$ for $L_{1}$ and $0.7 \mathrm{~dB}$ for $L_{2}$ at $850 \mathrm{~nm}$ ).

The wider the separation between the fibers, the higher the $L P_{01}$ extinction ratio $(17.1 \mathrm{~dB}$, $19 \mathrm{~dB}, 20.8 \mathrm{~dB}$ for $d=6,7$ and $8 \mu \mathrm{m}$ for a $10 \mathrm{~nm}$ bandwidth around $850 \mathrm{~nm}$ ) but the lower the $\mathrm{LP}_{11}$ extinction ratio $(22.7 \mathrm{~dB}, 21.5 \mathrm{~dB}$ and $20.4 \mathrm{~dB}$ respectively). The $d=8 \mu \mathrm{m}, L=56.58 \mathrm{~mm}$ symmetric coupler offers the best overall performance taking into account that the $\mathrm{LP}_{11}$ can be further attenuated at the corresponding output as previously commented for the asymmetric coupler.

\section{CONCLUSION}

In this letter, we propose the use of mode selective couplers at the band around $850 \mathrm{~nm}$ with standard single-mode fiber to achieve modal multiplexing with the $L P_{01}$ and $L P_{11}$ modes.

The best overall performance is obtained when an asymmetric coupler ( $d=6 \mu \mathrm{m} ; L=15.66 \mathrm{~mm}$ ) is selected as a mode converter and multiplexer (coupling efficiency $93.5 \%$ ) and a symmetric coupler $(d=8 \mu \mathrm{m} ; L=56.58 \mathrm{~mm}$ ) is used as demultiplexer (extinction ratio $<20.4 \mathrm{~dB}$ ) for a $10 \mathrm{~nm}$ bandwidth around $850 \mathrm{~nm}$. 


\section{REFERENCES}

[1] D. J. Richardson, J. M. Fini, and L. E. Nelson, "Space-division multiplexing in optical fibres," Nature Photon., vol. 7, pp. 354-362, May 2013.

[2] R. Ryf et al., "Mode-division multiplexing over $96 \mathrm{~km}$ of few-mode fiber using coherent 6 × 6 MIMO processing," J. Lightw. Technol., vol. 30, no. 4, pp. 521-531, Feb. 15, 2012.

[3] A. Li, X. Chen, A. A. Amin, and W. Shieh, "Fused fiber mode couplers for few-mode transmission," IEEE Photon. Technol. Lett., vol. 24, no. 21, pp. 1953-1956, Nov. 1, 2012.

[4] R. Ismaeel, T. Lee, B. Oduro, Y. Jung, and G. Brambilla, "All-fiber fused directional coupler for highly efficient spatial mode conversion," Opt. Exp., vol. 22, no. 10, pp. 11610-11619, May 2014.

[5] A. Li, J. Ye, X. Chen, and W. Shieh, "Fabrication of a low-loss fused fiber spatial mode coupler for few-mode transmission," IEEE Photon. Technol. Lett., vol. 25, no. 20, pp. 19851988, Oct. 15, 2013.

[6] N. Hanzawa, K. Saitoh, T. Sakamoto, T. Matsui, S. Tomita, and M. Koshiba, "Demonstration of mode-division multiplexing transmission over $10 \mathrm{~km}$ two-mode fiber with mode coupler," in Proc. OFC, Mar. 2011, pp. 1-3, paper OWA4.

[7] Physical Layer Specifications and Management Parameters for $40 \mathrm{~Gb} / \mathrm{s}$ and $100 \mathrm{~Gb} / \mathrm{s}$ Operation over Fiber Optic Cables, IEEE Standard 802.3bm-2015, 2015.

[8] S. Moon and D. Y. Kim, "Effective single-mode transmission at wavelengths shorter than the cutoff wavelength of an optical fiber," IEEE Photon. Technol. Lett., vol. 17, no. 12, pp. 2604-2606, Dec. 2005.

[9] Z. Tian, C. Chen, and D. V. Plant, "850-nm VCSEL transmission over standard single mode fiber using fiber mode filter," IEEE Photon. Technol. Lett., vol. 24, no. 5, pp. 368-370, Mar. 1, 2012.

[10] D. Marcuse, Theory of Dielectric Optical Waveguides. New York, NY, USA: Academic, 1974. 


\title{
3.2 Mode Conversion for Mode Division Multiplexing at $850 \mathrm{~nm}$ in Standard SMF
}

\section{BIBLIOGRAPHY RECORD}

- Authors: David Garcia-Rodriguez, Juan L. Corral and Roberto Llorente

- Publication: IEEE Photonics Technology Letters, vol. 29, no. 11

- Year: 2017

- Pages: 929-932

- Keywords: Mechanical mode converter, mode stripper, mode conversion, mode division multiplexing.

\begin{abstract}
A mechanical mode converter for mode division multiplexed systems over SSMF at $850 \mathrm{~nm}$ is proposed and evaluated experimentally by the transmission of OOK-modulated optical signals. The proposed mode converter is based on a periodic structure defined by the grating period $(\Lambda)$ parameter and the number of mode coupling points, $N$. A mechanical grating with $N=50$ points and a tunable grating period ( $\Lambda$ from 440 to $456 \mu \mathrm{m}$ ) has been designed offering a $124 \mathrm{~nm}$ tuning bandwidth. The $\mathrm{LP}_{01}$ mode to $\mathrm{LP}_{11}$ mode conversion has been experimentally assessed and a conversion efficiency of $89 \%$ has been achieved in the designed device. Optical transmission of OOK-modulated signals with 1.25 and $2.5 \mathrm{~Gb} / \mathrm{s}$ bitrates on $\mathrm{LP}_{01}$ mode or a converted $\mathrm{LP}_{11}$ mode is demonstrated achieving $\mathrm{BER}<10^{-9}$ after $1-\mathrm{km}$ optical transmission over SSMF at $850 \mathrm{~nm}$.
\end{abstract}




\section{INTRODUCTION}

The availability of cost effective vertical cavity surface emitting lasers (VCSEL's) at $850 \mathrm{~nm}$ combined with optimized designs of multimode fiber (MMF) have been stablished as a low cost solution for short range high speed optical data links [1,2]. The use of $850 \mathrm{~nm}$ VCSEL's for transmission over SSMF constitutes a promising low cost solution to improve the capacity and the link reach provided by MMF [3]. Using the $850 \mathrm{~nm}$ wavelength opens up the possibility to multiplex two linear polarization (LP) modes when standard single mode fiber (SSMF) is chosen as the transmission media, compared with more than 100 modes if multimode fiber (MMF) were employed. Fibers that propagates several modes are called "Few Mode Fibers" (FMF) or even "Two Mode Fibers" (TMF) to distinguish them from MMF, and can be designed to operate at short wavelengths [4]. Spatial-Division Multiplexing (SDM) or Mode-Division Multiplexing (MDM) are promising techniques to increase the capacity of fiber optic communication networks $[5,6]$. MDM increases the fiber capacity by means of the parallel transmission of different information through the available spatial modes at a specific wavelength. Specialty fibers or free-space transmission are usually considered on the MDM experiments instead of the ubiquitous SSMF $[4,7]$.

In any MDM system, mode converters and mode (de)multiplexers are required to combine and split the modes. Mode converters and multiplexers based on spatial light modulators or q-plates have been successfully used in MDM experiments $[4,7]$ but the devices based on fused fiber couplers offer a compact solution, with less losses and with direct integration with optical fiber. Both, symmetric (same fiber type) and asymmetric (different fiber type) fused fiber couplers have been proposed as mode converters and mode (de)multiplexers [8,9]. Asymmetric couplers are required in order to achieve mode conversion, whereas both asymmetric and symmetric couplers can be advantageously used as (de)multiplexer. Unfortunately, the fabrication tolerances required by the asymmetric couplers are more difficult to fulfill than the equivalent tolerances for the symmetric device. However, the use of symmetric couplers as (de)multiplexer requires an additional device to accomplish the mode conversion $\left(\mathrm{LP}_{01}\right.$ to $\left.L \mathrm{LP}_{11}\right)$ in the transmitter as shown in Figure 3.2.1 [8].

Mode conversion based on a mechanical grating for a MDM system operating at $1550 \mathrm{~nm}$ has been theoretically and experimentally proposed $[10,11]$. The mechanical mode converter transforms the $L P_{01}$ to $L P_{11}$ by applying pressure into the fiber [11]. This mechanical grating was designed to work at $1550 \mathrm{~nm}$ by selecting the appropriate grating period. However, the proposed system requires the use of a specialty fiber with a two mode behaviour at $1550 \mathrm{~nm}$. Instead, the use of SSMF as the optical media at the $850 \mathrm{~nm}$ band would benefit from the broad availability of SSMF-based infrastructure in current networks.

In this letter, the use of a mechanical converter for MDM in SSMF at $850 \mathrm{~nm}$ is first analysed. Following the analysis, a mode converter for operation at $850 \mathrm{~nm}$ in SSMF is designed and fabricated. Finally, the transmission performance employing OOK modulation is experimentally evaluated.

\section{MODEL}

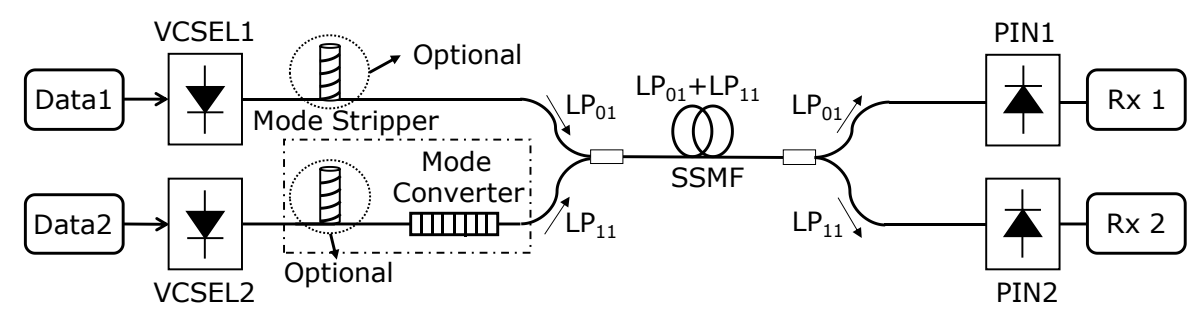

Figure 3.2.1. Scheme for mode-division multiplexing (MDM) at short wavelengths with SSMF as two-mode fiber (TMF) optical transmission media.

The MDM system including the mechanical mode converter is depicted in Figure 3.2.1. At the transmitter, two VCSEL sources are modulated by different data sources. The VCSEL's are 
coupled to SSMF patchcords. In general terms, both $L P_{01}$ and $L P_{11}$ could be excited in the fiber depending on the misalignment (lateral displacement or angle of incidence) of the laser light from the VCSEL to the core of the fiber. A mode stripper is placed after each VCSEL in order to eliminate any residual $L P_{11}$ content without reducing the $L P_{01}$ power. The $L P_{01}$ mode from the upper branch is directly sent to the fiber coupler acting as mode multiplexer. In the lower branch the $L P_{01}$ is converted to $\mathrm{LP}_{11}$ by using the mechanical mode converter and then sent to the fiber coupler when it is multiplexed with the optical signal coming from the upper branch. The multiplexed signals travel over the SSMF acting as a two-mode fiber at the $850 \mathrm{~nm}$ band. At the receiver, the $L P_{01}$ and $L P_{11}$ are demultiplexed by using the same device used as multiplexer in the transmitter.

(a)

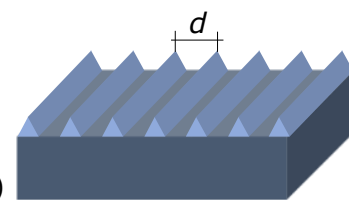

(b)

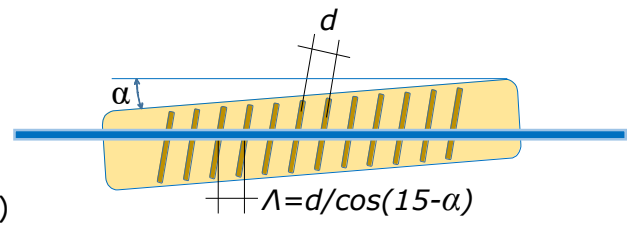

Figure 3.2.2. Mechanical Mode Converter: a) Structure, b) Design parameters.

A mechanical mode converter is a periodic structure defined by a grating period $(\Lambda)$ with $N$ coupling points that create a periodic perturbation on the optical fiber, as depicted in Figure 3.2.2. The grating period defines the wavelength where the efficiency to convert the $L P_{01}$ to $L P_{11}$ is maximum. The conversion efficiency can be expressed as [12]:

$$
\begin{gathered}
\frac{P_{11}}{P_{01}}=\frac{\sin ^{2}\left(\kappa \cdot L \sqrt{1+\left(\frac{\delta}{\kappa}\right)^{2}}\right)}{1+\left(\frac{\delta}{\kappa}\right)^{2}} \\
\delta=\frac{1}{2}\left\{\frac{2 \pi}{\lambda}\left(n_{L P 01}-n_{L P 11}\right)-\frac{2 \pi}{\Lambda}\right\}
\end{gathered}
$$

where $P_{01}$ is the power of the $L P_{01}$ mode, $P_{11}$ is the power of the $L P_{11}$ mode, $k$ is the coupling coefficient, $L$ is the grating length, $\delta$ is the detuning parameter, $\lambda$ is the wavelength, $n_{L P O 1}$ and $n_{L P 11}$ are the effective refractive indexes of the $L P_{01}$ and $L P_{11}$ modes and $\Lambda$ is the grating period. Equation (3.2.1) shows that the maximum coupling will take place when $\delta=0$. Therefore, from (3.2.2) it is possible to define the grating period as:

$$
\Lambda=\frac{\lambda}{n_{L P 01}-n_{L P 11}}
$$

In our case, the relative inclination of the grating axis with respect to the fiber ( $\alpha$ in Figure 3.2.2) can be adjusted by 15 degrees. Thus, the effective grating period, $\Lambda$, can be tuned between $d$ and $d / \cos (15)$ when the separation of the grating grooves is $d$. This variation in the effective grating period adds flexibility to adjust the modal converter to the fiber specific characteristics and the wavelength emitted by the laser.

Finally, for a periodic structure with equal pressure in each coupling point, the conversion efficiency would be [10]:

$$
\frac{P_{11}}{P_{01}}=\sin ^{2}\left(N \cdot \sin ^{-1}(\kappa)\right)
$$

The mechanical mode converter will be used with SMF-28 fiber from Corning Inc. ( $8.2 \mu \mathrm{m}$ core diameter, $\Delta=0.36 \%$ refractive index difference and $\mathrm{SiO}_{2}$ cladding). Sellmeier equation has been used to calculate the refractive and the modal effective indexes at $850 \mathrm{~nm}$. These are $n_{\text {core }}=1.4577, n_{\text {cladding }}=1.4525, n_{L P O 1}=1.4564$ and $n_{L P 11}=1.4545$. Finally, the optimum grating period to transform the $L P_{01}$ to the $L P_{11}$ at $850 \mathrm{~nm}$ is $\Lambda=447.36 \mu \mathrm{m}$. 


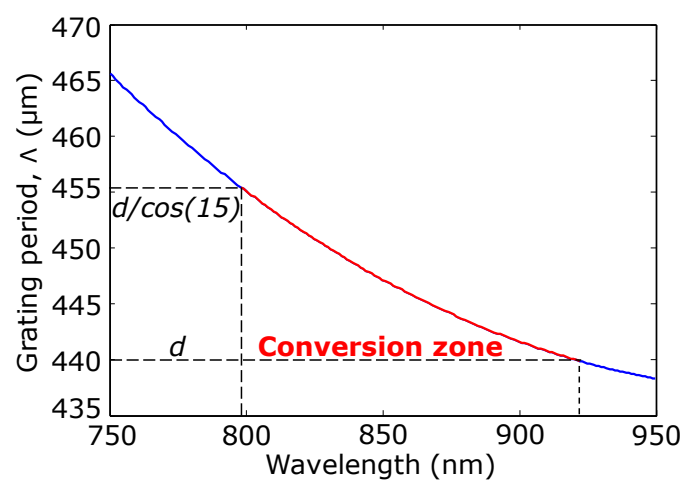

Figure 3.2.3. Grating period $(\Lambda)$ as a function of the wavelength.

According to (3.2.3) the grating period depends on the wavelength, both directly and indirectly through the wavelength dependence of the modal effective indexes. This dependence is shown in Figure 3.2.3.

A mechanical mode converter has been fabricated with $N=50, d=440 \mu \mathrm{m}$ and $L=2.2 \mathrm{~cm}$ in order to maximize the number of periods in the grating and to center the wavelength range of the device at $850 \mathrm{~nm}$. An effective grating period, $\Lambda$, between $440 \mu \mathrm{m}$ and $456 \mu \mathrm{m}$ can be obtained when $d=440 \mu \mathrm{m}$. Thus, this mode converter can transform $\mathrm{LP}_{01}$ to $\mathrm{LP}_{11}$ in a $124 \mathrm{~nm}$ bandwidth around $850 \mathrm{~nm}$ (from $798 \mathrm{~nm}$ to $922 \mathrm{~nm}$, marked as conversion zone in Figure 3.2.3) if SMF-28 fiber is used.

\section{MODE CONVERSION EFFICIENCY}

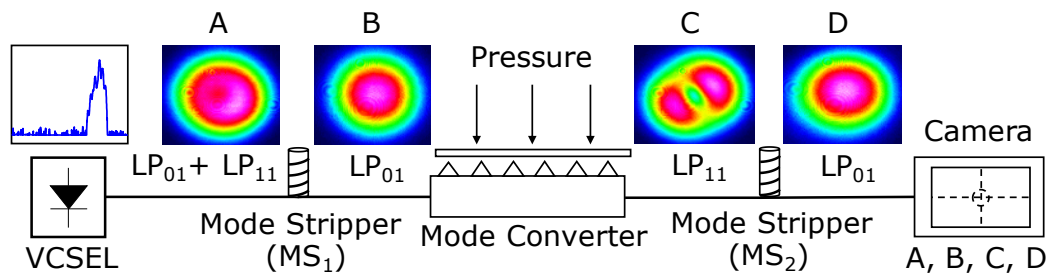

Figure 3.2.4. Setup for the conversion from $L P_{01}$ to $L P_{11}$.

The mechanical mode converter transforms a particular mode to the next higher order mode through the grating period. The setup depicted in Figure 3.2.4 has been used to experimentally demonstrate the conversion efficiency of the mechanical mode converter previously designed. The optical signal generated in an $850 \mathrm{~nm}$ VCSEL is coupled to a patchcord of bare SMF-28 fiber. The output of the VCSEL will be a combination of both $L P_{01}$ and $L P_{11}$ modes. The two linear polarization (LP) modes coming from the VCSEL are guided to the first mode stripper $\left(M S_{1}\right)$ to erase the $L P_{11}$ content and obtain a pure $L P_{01}$ mode. The mechanical mode converter transforms the $L P_{01}$ to the $L P_{11}$ mode. A CCD camera Beam Profiler will be placed in the positions $A, B, C$ or $D$ in order to visualize the modal profile. The camera in the $C$ position permits to properly adjust the pressure at the mechanical mode converter measuring the power obtained and visualizing the modal profile.

Once the $L P_{11}$ is obtained, a second mode stripper $\left(M S_{2}\right)$ is used to erase the converted $L P_{11}$. The $L P_{11}$ power content is determined as the power difference between points $B-D$, as the $M S_{2}$ will only remove the $\mathrm{LP}_{11}$ mode. Finally, the conversion efficiency will be obtained as the ratio between this result and the total optical power after mode conversion (point $\mathrm{C}$ ). The designed mechanical grating will be placed on a stand as described in [10] in order to manually adjust the pressure level applied on the fiber and the relative angle between the grating and the fiber. The periodic perturbation produced by the grating will transform the $L P_{01}$ mode to the $L P_{11}$ mode. A low level pressure is required in the mechanical mode converter to achieve the mode transformation. If the pressure is too high, the $\mathrm{LP}_{01}$ power will be attenuated and the conversion efficiency will be reduced. It is important to point out that the fiber used in the setup is SMF-28 fiber with a $250 \mu \mathrm{m}$ cladding and no additional coating. The thin cladding 
makes it easier to apply the pressure produced by the mechanical mode converter to the fiber core without breaking the fiber.

The mode strippers used in the setup are made by coiling the SSMF fiber around a cylinder of a certain diameter in order to erase the $\mathrm{LP}_{11}$ mode without losing the power content of the $\mathrm{LP} \mathrm{P}_{01}$ mode. Different diameters (from $0.6 \mathrm{~cm}$ to $1.2 \mathrm{~cm}$ ) have been studied, obtaining an optimum size of $1.0 \mathrm{~cm}$, which eliminates completely the $L P_{11}$ mode after a few loops without damaging the $\mathrm{LP}_{01}$ mode (losses $<0.01 \mathrm{~dB}$ ). The mechanical mode converter configuration (pressure level, and relative angle between fiber and grating) were selected by using the CCD camera at the output of the mode converter and choosing the purest $\mathrm{LP}_{11}$ mode profile as shown in Figure 3.2.6(b).

It has to be taken into account that the mode converter induces insertion losses around 0.2-0.5 dB depending on the pressure level applied on the fiber core. For the optimum mode converter configuration these losses are equal to $0.5 \mathrm{~dB}$.

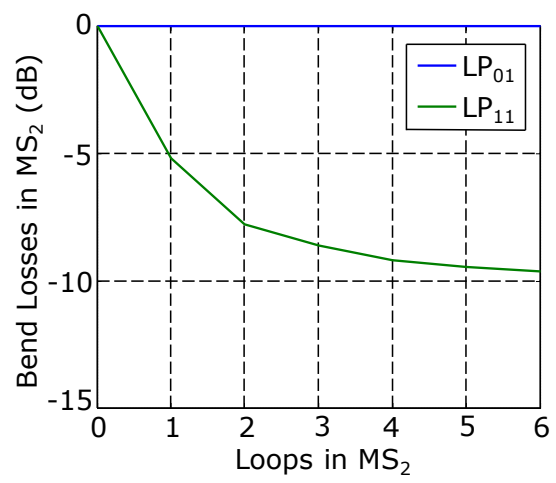

Figure 3.2.5. Bend losses in the $\mathrm{MS}_{2}$ as a function of the loop number.

In order to measure the conversion efficiency, the difference in optical power between the points B-D in Figure 3.2.4 must be measured when the $\mathrm{MS}_{2}$ mode stripper removes all the $\mathrm{LP}_{11}$ mode content. The number of loops applied to the fiber in this mode stripper will define the attenuation level suffered by the $\mathrm{LP}_{11}$ mode. As the number of loops increases, the power level in point $D$ is reduced until the $L P_{11}$ is fully removed and the residual $L P_{01}$ mode content will be the only term to be measured. In Figure 3.2.5 the optical attenuation between points B-D is shown for 0 to 6 loops in the $\mathrm{MS}_{2}$ mode stripper $(1.0 \mathrm{~cm}$ diameter). From these results, it is clear that after 6 loops the $\mathrm{LP}_{11}$ content is fully removed. The residual $L P_{01}$ content is shown to be $9.5 \mathrm{~dB}$ below the $L \mathrm{P}_{11}$ mode content, which corresponds to a $L \mathrm{P}_{01}$ to $L \mathrm{P}_{11}$ mode conversion efficiency of $89 \%$ and a coupling coefficient of $K=0.025$.

\section{MODE TRANSMISSION EXPERIMENT}

The MAX3996 evaluation board from Maxim Integrated will be used to assess the data transmission performance of the $\mathrm{LP}_{01}$ and $\mathrm{LP}_{11}$ modes over SSMF fiber at $850 \mathrm{~nm}$. This board is equipped with the MAX3996 VCSEL laser driver and a maximum data nm rate of $2.5 \mathrm{~Gb} / \mathrm{s}$ can be selected. An $850 \mathrm{~nm}$ VCSEL with an electrical bandwidth greater than $1 \mathrm{GHz}$ has been added to the evaluation board. This VCSEL is coupled to a SSMF pigtail with an optical output power of $-13 \mathrm{dBm}$.

The use of the $850 \mathrm{~nm}$ band in SSMF is aimed at short reach links due to the high losses $(\sim 2 \mathrm{~dB} / \mathrm{km})$ of the fiber at this wavelength. Due to an additional limitation on the output power coupled to the SMF-28 from the available $850 \mathrm{~nm}$ VCSEL, data transmission measurements were limited to $1 \mathrm{~km}$ reach. 

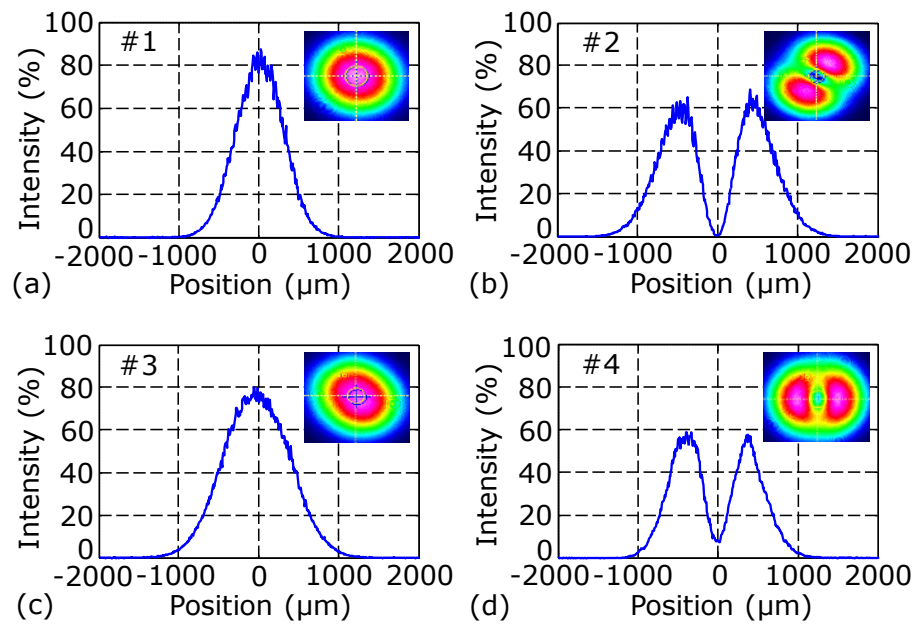

Figure 3.2.6. Modal profiles in SSMF: a) $L P_{01}$ mode in $B 2 B$ transmission, $\left.b\right) L P_{11}$ mode $B 2 B$ transmission, c) $L P_{01}$ mode after $1 \mathrm{~km}$, d) $L P_{11}$ mode after $1 \mathrm{~km}$.

In the first experiment, the evolution of the $L P_{01}$ and $L P_{11}$ modes through the $1 \mathrm{~km}$ link has been assessed by transmitting the unmodulated optical signal alternatively with and without pressure applied to the mode converter. The corresponding mode profiles for $L P_{01}$ and $L P_{11}$ are depicted in Figure 3.2.6, where the results for back-to-back transmission (pictures \#1 and \#2) and along $1 \mathrm{~km}$ of fiber (pictures \#3 and \#4) are compared. The mode profiles are maintained at the output of the link and the viability of the MDM system at $850 \mathrm{~nm}$ over SSMF would be ensured if both modes were transmitted simultaneously and the proper mode (de)multiplexers were available. Both modes show an excellent stability after $1 \mathrm{~km}$ long transmission. In both cases, there is a minor interference of the alternate mode when the mode profile is taken after $1 \mathrm{~km}$ of fiber. The $L \mathrm{P}_{01}$ mode profile after $1 \mathrm{~km}$ is slightly broadened if compared to the $\mathrm{B} 2 \mathrm{~B}$ case due to a minimal $L P_{11}$ power level. In the $L P_{11}$ case, the intensity level just in the center of the fiber is not exactly zero after $1 \mathrm{~km}$ of fiber what corresponds to the presence of a reduced level of the $\mathrm{LP}_{01}$ mode. If we compare the modal profiles in Figure 3.2.6(b) and Figure 3.2.6(d), the interference of the $L P_{01}$ mode in Figure 3.2.6(d) corresponds to a relative intensity of the $L P_{01}$ mode $10 \mathrm{~dB}$ below the intensity of the $\mathrm{LP}_{11}$ due to the modal coupling in the optical connector. This behavior is due to the mode coupling ( $L P_{01}$ to $L P_{11}$ and $L P_{11}$ to $\left.L P_{01}\right)$ induced by the connector between the bare fiber at the mode converter and the fiber span as it would happen with any connector in any TMF/FMF link. The elimination of this connector by using the same fiber in the converter and in the fiber span would avoid any potential mode crosstalk. No mode crosstalk is expected to be due to the mode coupling along the fiber as the difference between the effective indexes of both modes is $1.310^{-3}$ [13].

In order to test the link capability along $1 \mathrm{~km}$ we will transmit the individual modes modulated by an OOK signal with a $2^{31}-1$ pseudo random bit sequence (PRBS) at $1.25 \mathrm{~Gb} / \mathrm{s}$ and $2.5 \mathrm{~Gb} / \mathrm{s}$. The evaluation can be done through the measurement of the quality factor $(Q)$, which determines the quality of the eye diagram and it is directly related to the Bit Error Rate (BER).

Figure 3.2.7 shows the eye diagrams for the $L P_{01}$ and $L P_{11}$ modes at a bit rate of $1.25 \mathrm{~Gb} / \mathrm{s}$. For the $\mathrm{B} 2 \mathrm{~B}$ case, both modes have an open eye diagram with quality factors around $Q \approx 19$ and $Q \approx 8$, respectively. This difference between quality factors is due to the conversion losses from $L P_{01}$ to $L P_{11}$; however, it is important to mention that both signals have a quality factor higher than $Q=6$, corresponding to a $B E R=10^{-9}$. After the $1 \mathrm{~km}$ transmission, the quality of the eye diagrams have been slightly reduced due to the attenuation of the fiber and the influence of the fiber connectors. Anyway, the final $Q$ value for the $L P_{01}$ and $L P_{11}$ is approximately $Q \approx 6.8$. In this case, both modes can be transmitted and received correctly at $1.25 \mathrm{~Gb} / \mathrm{s}$ along $1 \mathrm{~km}$. 

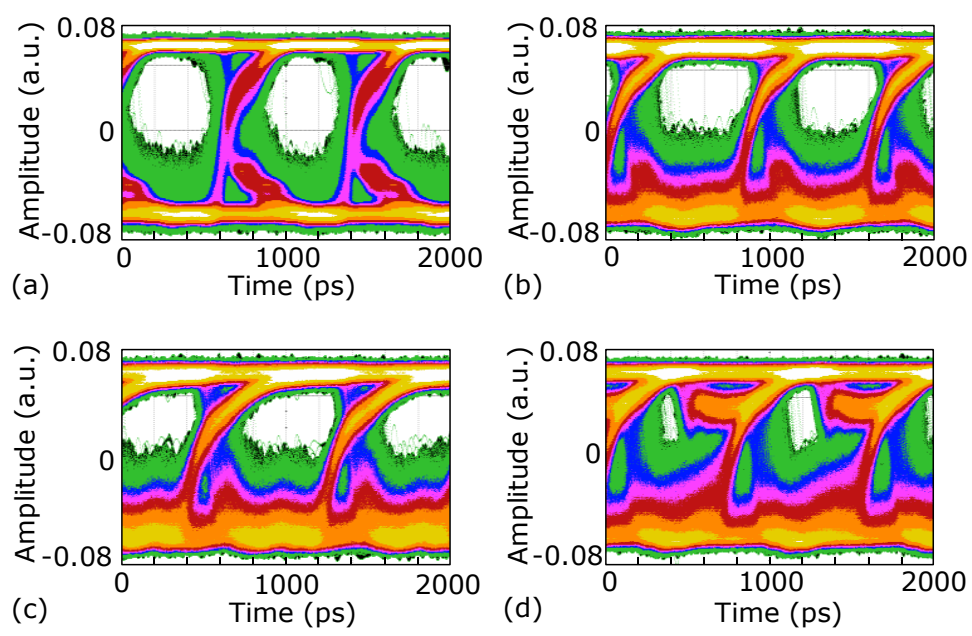

Figure 3.2.7. Eye diagram for the $L P_{01}$ and $L P_{11}$ modes at $1.25 \mathrm{~Gb} / \mathrm{s}$ : a) $\left.\left.B 2 B L P_{01}, b\right) B 2 B L P_{11}, c\right) 1 \mathrm{~km}$ $L P_{01}$ and d) $1 \mathrm{~km} \mathrm{LP} 11$.

Figure 3.2.8 shows the same transmission as the Figure 3.2.7 but changing the bit rate to $2.5 \mathrm{~Gb} / \mathrm{s}$. It is important to point out that the bandwidth limitation of the VCSEL used for the experiments is affecting the transmission even for the B2B case as shown in Figure 3.2.8(a) and Figure 3.2.8(b), with a quality factors around $Q \approx 9$ and $Q \approx 8$, respectively. After $1 \mathrm{~km}$ transmission, the $\mathrm{LP}_{01}$ and $\mathrm{LP}_{11}$ suffer almost the same distortion at $2.5 \mathrm{~Gb} / \mathrm{s}$ that at $1.25 \mathrm{~Gb} / \mathrm{s}$. The resulting quality factors for the $\mathrm{LP}_{01}$ and $\mathrm{LP}_{11}$ after $1 \mathrm{~km}$ are both around $Q \approx 6.2$, again higher than $Q=6$.
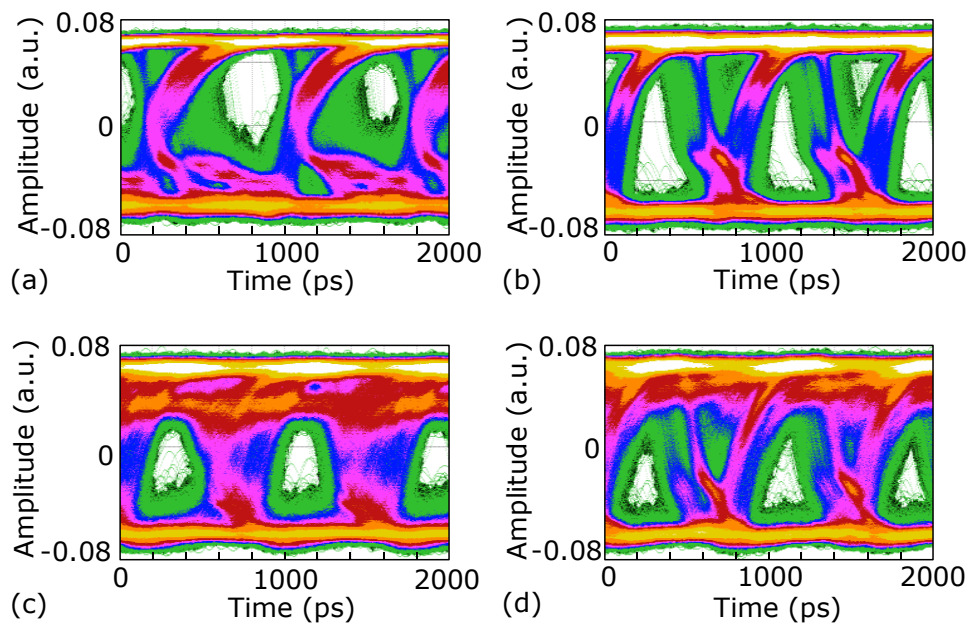

Figure 3.2.8. Eye diagram for the $L P_{01}$ and $L P_{11}$ modes at $2.5 \mathrm{~Gb} / \mathrm{s}$ : a) $B 2 B L P_{01}$, b) $\left.B 2 B L P_{11}, c\right) 1 \mathrm{~km}$ $L P_{01}$ and d) $1 \mathrm{~km} L P_{11}$.

\section{CONCLUSION}

In this letter, we have proposed and evaluated experimentally the use of a mechanical mode converter at $850 \mathrm{~nm}$ to achieve mode transformation from LP 01 to $\mathrm{LP}_{11}$ in SSMF.

The design of the mechanical grating is centered at $850 \mathrm{~nm}$ but it can be tuned in a $124 \mathrm{~nm}$ bandwidth. The tunability can be advantageously used to adapt the mode converter to tolerances in fiber specifications or laser performance. The mechanical mode converter has been shown to offer $89 \% L_{01}$ to $L_{11}$ conversion efficiency. The stability of both modes (LP ${ }_{01}$ and converted $\mathrm{LP}_{11}$ ) has been proved in a $1 \mathrm{~km}$ SSMF link.

Finally, data transmission over $1 \mathrm{~km}$ at $1.25 \mathrm{~Gb} / \mathrm{s}$ and $2.5 \mathrm{~Gb} / \mathrm{s}$ OOK signal was achieved obtaining a quality factor higher than $Q=6\left(B E R=10^{-9}\right)$ for both modes. The link distance and achieved bitrate were limited by the reduced output power and modulation bandwidth of the VCSEL source. 


\section{REFERENCES}

[1] J. A. Tatum et al., "VCSEL-based interconnects for current and future data centers," ]. Lightw. Technol., vol. 33, no. 4, pp. 727-732, Feb. 15, 2015.

[2] D. Vez et al., "Packaged $850 \mathrm{~nm}$ vertical-cavity surface-emitting lasers as low-cost optical sources for transparent fibre-optic links," in Proc. 33rd Eur. Microw. Conf., Munich, Germany, Oct. 2003, pp. 611-614.

[3] Z. Tian, C. Chen, and D. V. Plant, "850-nm VCSEL transmission over standard single mode fiber using fiber mode filter," IEEE Photon. Technol. Lett., vol. 24, no. 5, pp. 368-370, Mar. 1, 2012.

[4] G. Milione, E. Ip, M.-J. Li, J. Stone, G. Peng, and T. Wang, "Mode crosstalk matrix measurement of a $1 \mathrm{~km}$ elliptical core few-mode optical fiber," Opt. Lett., vol. 41, no. 12, pp. 2755-2758, 2016.

[5] D. Richardson, J. Fini, and L. Nelson, "Space-division multiplexing in optical fibres," Nature Photon., vol. 7, no. 5, pp. 354-362, May 2013.

[6] E. Ip et al., "SDM transmission of real-time 10 GbE traffic using commercial SFP+ transceivers over $0.5 \mathrm{~km}$ elliptical-core few-mode fiber," Opt. Exp., vol. 23, no. 13, pp. 1712017126, 2015.

[7] G. Milione et al., " $4 \times 20 \mathrm{Gbit} / \mathrm{s}$ mode division multiplexing over free space using vector modes and a q-plate mode (de)multiplexer," Opt. Lett., vol. 40, no. 9, pp. 1980-1983, 2015.

[8] N. Hanzawa, K. Saitoh, T. Sakamoto, T. Matsui, S. Tomita, and M. Koshiba, "Demonstration of mode-division multiplexing transmission over $10 \mathrm{~km}$ two-mode fiber with mode coupler," in Proc. OFC, Mar. 2011, pp. 1-3.

[9] J. L. Corral, D. Garcia-Rodriguez, and R. Llorente, "Mode-selective couplers for two mode transmission at $850 \mathrm{~nm}$ in standard SMF," IEEE Photon. Technol. Lett., vol. 28, no. 4, pp. 425428, Feb. 15, 2016.

[10] I. Giles, A. Obeysekara, R. Chenm, D. Giles, F. Poletti, and D. Richardson, "Fiber LPG mode converters and mode selection technique for multimode SDM," IEEE Photon. Technol. Lett., vol. 24, no. 21, pp. 1922-1925, Nov. 1, 2012.

[11] P. Pradhan, D. Sengupta, H. Rehouma, V. Francois, C. Tremblay, and B. Ung, "Excitation of vector modes in few-mode fiber using wire-based mechanical long period fiber grating," in Proc. IEEE Photonics North, Jun. 2015, p. 1.

[12] A. M. Vengsarkar, P. J. Lemaire, J. B. Judkins, V. Bhatia, T. Erdogan, and J. E. Sipe, "Long-period fiber gratings as band-rejection filters," J. Lightw. Technol., vol. 14, no. 1, pp. 58-65, Jan. 1, 1996.

[13] F. Yaman, N. Bai, B. Zhu, T. Wang, and G. Li, "Long distance transmission in few mode fibers," Opt. Exp., vol. 18, no. 12, pp. 13250-13257, 2010. 


\title{
3.3 Modal Selectivity at $850 \mathrm{~nm}$ employing Standard Single- Mode Couplers: Theory and Experimental Demonstration
}

\section{BIBLIOGRAPHY RECORD}

- Authors: David Garcia-Rodriguez, Maria Morant, Juan L. Corral and Roberto Llorente

- Publication: Submitted to Optics Communication

- Year: TBD

- Pages: TBD

- OCIS codes: Optical fiber coupler, mode multiplexer, mode division multiplexing, few-mode fiber.

\begin{abstract}
In this paper, we evaluate experimentally and analyze theoretically and by simulation the demultiplexing of $L P_{01}$ and $L P_{11}$ modes in a $850 \mathrm{~nm}$ few mode optical link when a standard single-mode coupler theoretically designed for $1550 \mathrm{~nm}$ operation is used. The simulation analysis indicates a feasible conversion efficiency higher than $98 \%$ for the $L P_{01}$ mode and $100 \%$ efficiency for $\mathrm{LP}_{11}$ mode. These results are experimentally validated considering different off the shelf optical couplers configured in 99:1, 90:10, 80:20 and 60:40 coupling ratios. Experimental analysis of the optical couplers demonstrate adequate functionality in correspondence with the theoretical analysis, exhibiting a good near-field pattern and modal profile for the $L P_{01}$ and $L P_{11}$ modes. The mode purity observed was $100 \%$ for the $L P_{01}$ mode, and higher than $90 \%$ for the $L P_{11}$ mode. The 90:10 optical coupler configuration shows best mode purity with the minimal insertion losses.
\end{abstract}




\section{INTRODUCTION}

In the last decade, due to the huge amount of internet traffic, researchers have focused their efforts to increase the transmission capacity of the optical systems. Five physical dimensions can be employed to characterize the optical data transmitted (time, wavelength, quadrature, polarization and spatial propagation mode) [1]. Space division multiplexing (SDM) or mode division multiplexing (MDM) are the key to surpass the capacity limit of the optical fiber, either employing multicore fibers or different optical modes in the optical fiber [2-6]. The fibers that support more than 100 modes are called multimode fibers (core/cladding diameters of $50 / 125 \mu \mathrm{m}$ and $62.5 / 125 \mu \mathrm{m}$ ) but the relative delay between the different modes introduce a huge restriction in long transmission links. For this reason, the fibers supporting a small number of optical modes have attracted a lot of interest and are now recognized as one of the most promising technologies to increase the transmission capacity.

In any MDM system, mode converters and mode (de)multiplexers are the key to combine/divide the different modulated signals [7]. Recently, the use of a fiber based directional coupler for a MDM system at $1550 \mathrm{~nm}$ has been theoretically [8] and experimentally $[9,10]$ considered. However, the proposed system is based on the use of a specialty fiber with a two mode behavior at $1550 \mathrm{~nm}$.

The $L P_{01}$ and $L P_{11}$ modes propagate when standard single mode fiber (SSMF), like SMF-28 with $8.2 / 125 \mu \mathrm{m}$ core/cladding diameters, at $850 \mathrm{~nm}$ is employed, making a low cost solution without the need of any extra devices or design [7]. The optical modes have a high differential mode delay (DMD) value ensuring a negligible modal coupling along the SSMF link [11]. This band opens up the possibility of using low cost devices such as $10 \mathrm{~Gb} / \mathrm{s}$ VCSEL [12].

Previously, the use of mode filters in a SSMF at $850 \mathrm{~nm}$ wavelength without taking advantage of the MDM benefits have been proposed $[13,14]$. Besides these results, in order to increase the SSMF capacity using MDM at $850 \mathrm{~nm}$ band, a custom fiber coupler or mechanical mode converter has been theoretically [7] and experimentally [15] proposed and demonstrated. On one hand, the asymmetric fiber couplers permit to convert the $L P_{01}$ mode into the $\mathrm{LP}_{11}$ mode with the added complexity of achieving the stringent fabrication tolerances required. On the other hand, a mechanical mode converter can be used to obtain a pure $\mathrm{LP}_{11}$ mode adding extra losses to the MDM link and requiring additional manual adjustment.

Additionally, a preliminary simulation and experimental demonstration of the correct demultiplexing of the 90:10 optical coupler has been reported [16]. The new study here presented is exhaustively performed taking into account the theory and experimental results in order to achieve the best performance.

In this letter, a comprehensive study of the $L P_{01}$ and $L P_{11}$ modes demultiplexing in commercially available standard single mode couplers designed to operate at the $1550 \mathrm{~nm}$ band, and acting as mode multiplexer/demultiplexer at $850 \mathrm{~nm}$, is presented. Both the simulation analysis of the coupling efficiency in the $1550 \mathrm{~nm}$ and $850 \mathrm{~nm}$ bands and the experimental results of modal purity and insertion losses are considered.

\section{THEORETICAL STUDY}

The basic concept of the MDM link is depicted in Figure 3.3.1. It consists of two lasers emitting at $850 \mathrm{~nm}$ propagating the $\mathrm{LP}_{01}$ and $\mathrm{LP}_{11}$ modes in a standard single-mode fiber (SSMF). As the SSMF fiber behaves effectively as a two-mode fiber (TMF) at $850 \mathrm{~nm}$, a bimodal optical link is obtained. Both modes will be coupled to the TMF using a commercial $2 \times 1$ optical coupler originally designed to operate at the $1550 \mathrm{~nm}$ wavelength. The behavior of the optical coupler at $850 \mathrm{~nm}$ operation will allow to multiplex the $L P_{01}$ mode from the upper branch with the $\mathrm{LP}_{11}$ mode from the lower branch to the common output. The residual content of the $\mathrm{LP}_{11}$ mode at the upper branch and the $\mathrm{LP}_{01}$ mode at the lower branch will be eventually diverted to the other coupler output and discarded. At the receiver side, the same fiber coupler will act as a mode demultiplexer and each mode will be routed to the corresponding photodiode. 


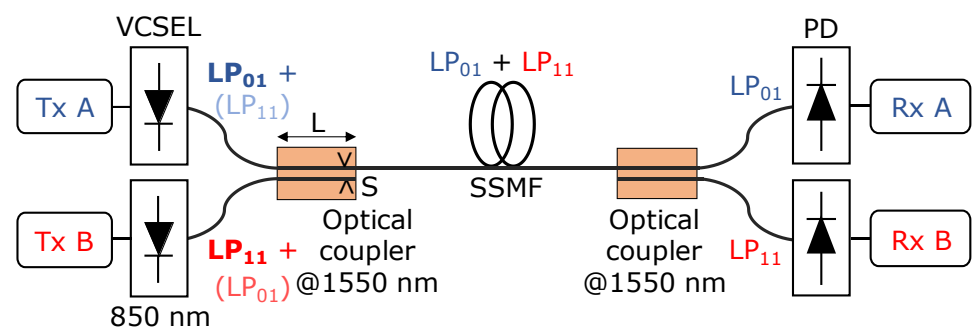

Figure 3.3.1. MDM transmission using standard single-mode couplers as multiplexer/demultiplexer at $850 \mathrm{~nm}$.

\section{COUPLING THEORY}

According to two mode coupling theory, the optical fields along each optical fiber can be expressed as:

$$
\begin{aligned}
& \vec{E}^{A}(x, y, z, t)=A(z) \vec{\varepsilon}_{m n}^{A}(x, y) e^{j\left(\beta_{m n}^{A} z-\omega t\right)} \\
& \vec{E}^{B}(x, y, z, t)=B(z) \vec{\varepsilon}_{p q}^{B}(x, y) e^{j\left(\beta_{p q}^{B} z-\omega t\right)}
\end{aligned}
$$

Where $\vec{\varepsilon}_{m n}^{A}(x, y)$ and $\vec{\varepsilon}_{p q}^{B}(x, y)$ are the modal profiles in each optical fiber ( $m n$ in fiber A and $p q$ in fiber $\mathrm{B}$ ) while $\beta_{m n}^{A}$ and $\beta_{p q}^{A}$ are the propagation constants for both modes, respectively. Each individual mode is excited with their corresponding modal profile and propagation constant while the local modal profiles changes with the variation of the complex envelopes, $A(z)$ and $B(z)$, along the direction of propagation.

If we consider a simple case when power is launched only into mode $a$ at $L=0$, the optical power in both outputs (direct (A) and coupled (B) paths) as a function of the input power $P_{a}(0)$ is [16]:

$$
\begin{gathered}
\frac{P_{a}(L)}{P_{a}(0)}=\left|\frac{A(L)}{A(0)}\right|^{2}=\left(\frac{\kappa_{a b} \kappa_{b a}}{\beta_{c}^{2}}\right) \cos ^{2}\left(\beta_{c} L\right)+\left(\frac{\delta}{\beta_{c}}\right)^{2} \\
\frac{P_{b}(L)}{P_{a}(0)}=\left|\frac{B(L)}{A(0)}\right|^{2}=\frac{\left|\kappa_{b a}\right|^{2}}{\beta_{c}^{2}} \sin ^{2}\left(\beta_{c} L\right) \\
\delta=\frac{\left(\beta_{p q}^{B}+\kappa_{b b}\right)-\left(\beta_{m n}^{A}+\kappa_{a a}\right)}{2} \\
\beta_{c}=\sqrt{\kappa_{a b} \kappa_{b a}+\delta^{2}}
\end{gathered}
$$

where $\kappa_{a a}, \kappa_{b b} . \kappa_{a b}$ and $\kappa_{b a}$ are the self-coupling and mutual coupling coefficients, respectively, and $\delta$ is the phase mismatch. Finally, the coupling efficiency for a length $L$ is

$$
\eta=\frac{P_{b}(L)}{P_{a}(0)}=\frac{\left|\kappa_{b a}\right|^{2}}{\beta_{c}^{2}} \sin ^{2}\left(\beta_{c} L\right)
$$

Thus, power is exchanged periodically between both modes with a coupling length

$$
L_{c}=\frac{\pi}{2 \beta_{c}}
$$

where the maximum coupling is achieved when the phase mismatch is zero $(\delta=0)$. In this case, the propagation constants (and the refractive indexes) of both modes in the coupler will be the same. 


\section{DEVICE STRUCTURE}

The optical coupler is employed as a mode multiplexer and mode demultiplexer of the $\mathrm{LP}_{01}$ and $\mathrm{LP}_{11}$ modes at $850 \mathrm{~nm}$, as it is shown in Figure 3.3.2. The structure is defined by the diameter of the fiber $(D)$, the separation between cores $(S)$ and the coupling length $(L)$

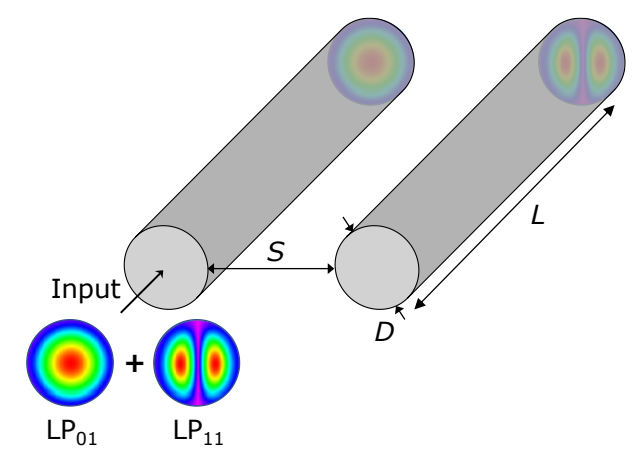

Figure 3.3.2. 3D sketch of the structure of the fiber coupler mode (de)multiplexer.

The fiber to be used for the optical coupler is the standard single mode fiber; namely, SMF-28 from Corning Inc. ( $D=8.2 \mu \mathrm{m}$ core diameter, $\Lambda=0.36 \%$ refractive index difference and ${\mathrm{a} \mathrm{SiO}_{2}}_{2}$ cladding). At $850 \mathrm{~nm}$, only two linear polarization (LP) modes are supported in SMF-28, behaving as a TMF. The refractive indexes are calculated employing the Sellmeier equation, obtaining a $n_{\text {core }}=1.4577$ and $n_{\text {cladding }}=1.4525$. Also, the modal effective indexes are calculated with the three dimensional (3D) beam propagation method (3D-BPM) with values of $n_{L P 01}=1.4564$ and $n_{L P 11}=1.4545$.

\section{SIMULATION ANALYSIS}

The optical coupler has been simulated at $850 \mathrm{~nm}$ for different gap, $S(\mu \mathrm{m})$, and coupling length, $L(\mathrm{~mm})$ values. The maximum coupling efficiency is obtained when the equation (3.3.8) is fulfilled; therefore, for several gaps and lengths different coupling efficiencies will be achieved, as can be seen in Figure 3.3.3 for the $\mathrm{LP}_{11}$ mode.

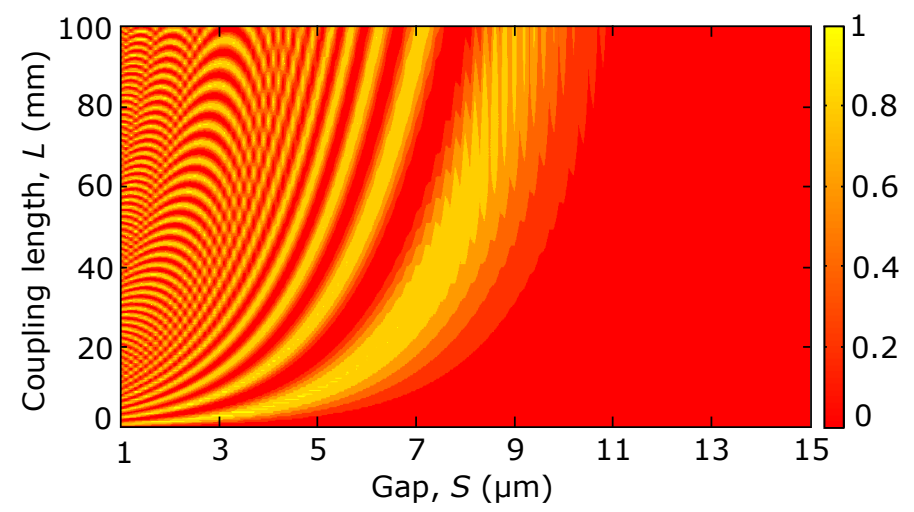

Figure 3.3.3. $L P_{11}$ coupling efficiency in a SMF-28 fiber coupler as a function of the coupler gap and length.

If the gap increases, the mutual coupling coefficients are reduced and, therefore, the optical coupler requires longer coupling lengths to achieve the maximum coupling efficiency.

When the optical coupler acts as a (de)multiplexer each mode is split to different outputs. The $L P_{01}$ mode remains in the straight path whereas the $\mathrm{LP}_{11}$ mode is coupled to the cross path. Different coupler configurations $(S, D, L)$ can be selected in order to achieve a perfect demultiplexing of the $\mathrm{LP}_{11}$ mode. It can be stated from Figure 3.3.3 that the maximum separation between cores for a maximum coupling of the $L P_{11}$ mode is around $9 \mu \mathrm{m}(90 \mathrm{~mm}$ coupling length (yellow color band)). For wider separations, the mutual coupling coefficients are sufficiently separated and a very large length is needed to couple the $\mathrm{LP}_{11}$ mode. Thus, the commercial couplers designed to operate at $1550 \mathrm{~nm}$ will have a separation between cores 
shorter than $9 \mu \mathrm{m}$. Typically, commercial optical couplers present coupling lengths of few centimeters [17].
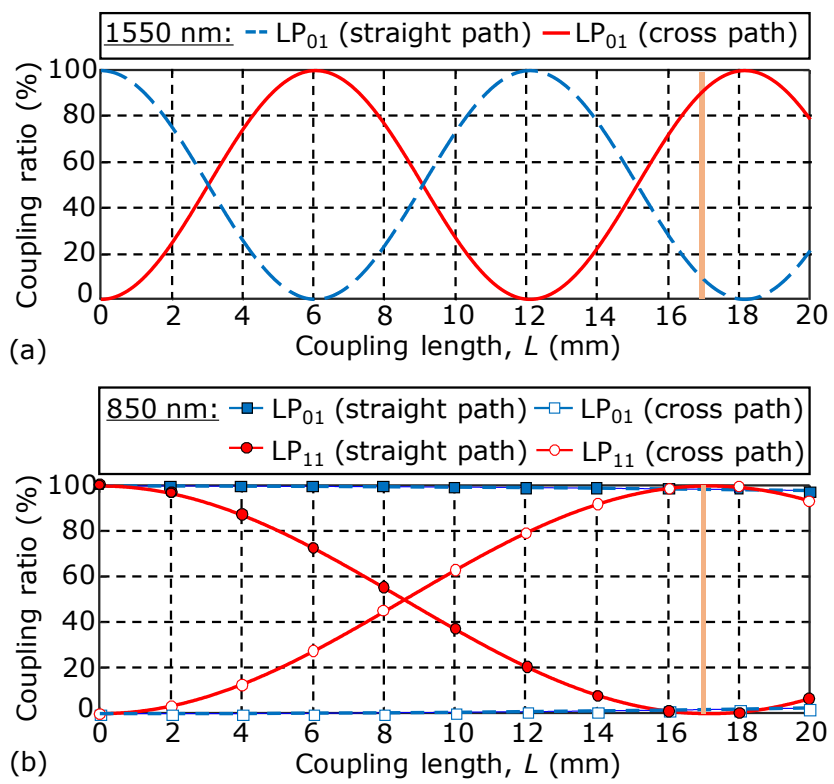

Figure 3.3.4. Different coupling efficiencies when both $L P_{01}$ and $L P_{11}$ modes are excited and the $L P_{11}$ mode is demultiplexed at the cross path.

The simulation analysis was performed taking into account two different wavelengths, $1550 \mathrm{~nm}$ and $850 \mathrm{~nm}$, in order to evaluate the behavior of the device in the design and operation wavelengths. To maximize the response of the optical coupler, the separation between cores is fixed at $S=6 \mu \mathrm{m}$, with a coupling length up to $L=20 \mathrm{~mm}$.

Figure 3.3.4 shows the simulation results of the coupling efficiency when the optical coupler acts as demultiplexer. For the $1550 \mathrm{~nm}$ operation, only the $L \mathrm{P}_{01}$ mode is propagated, as it can be seen in Figure 3.3.4(a). In this case, the maximum coupling efficiency from the straight path to the cross path takes place twice along the evaluated coupling length of $L=20 \mathrm{~mm}$. Thus, if we focus the attention in the highlighted region, a coupling ratio of 90:10 is obtained when the coupling length is $L=17 \mathrm{~mm}$. Figure 3.3.4(b) shows the coupling efficiency of the $L P_{01}$ and $L_{11}$ modes operating at $850 \mathrm{~nm}$. For the same highlighted region, $L=17 \mathrm{~mm}$, the $\mathrm{LP}_{01}$ mode obtained a coupling efficiency of $98 \%$ in the straight path and $1.76 \%$ in the cross path (that corresponds with an extinction ratio of $17.55 \mathrm{~dB}$ ). However, the $\mathrm{LP}_{11}$ mode reaches a $100 \%$ in the cross path (for the same region) and $0 \%$ in the cross path. It can be stated that the commercial 90:10 optical coupler designed for $1550 \mathrm{~nm}$ can be used as multiplexer/demultiplexer of the $L P_{01}$ and $L P_{11}$ modes over SSMF fiber operating at $850 \mathrm{~nm}$.

The simulation results are based on real fabrication parameters for a typical 90:10 optical coupler at $1550 \mathrm{~nm}$, however, depending on the manufacturer and the fabrication method used, the coupler performance at $850 \mathrm{~nm}$ may differ.

\section{EXPERIMENTAL VALIDATION}

The schematic diagram of the experimental setup to obtain the near-field patterns of the $\mathrm{LP}_{01}$ and $\mathrm{LP}_{11}$ modes is shown in the Figure 3.3.5. The vertical cavity surface emitting laser (VCSEL) transmitting at $850 \mathrm{~nm}$ and coupled to a SSMF pigtail propagates the $\mathrm{LP}_{01}$ and $\mathrm{LP}_{11}$ modes. When both modes enter the optical coupler they are demultiplexed in different output ports ( $L P_{01}$ in output $A$ and $L P_{11}$ in output $B$ ). The optical mode at each output will be visualized independently by manually selecting the corresponding output. For any single output, the mode is guided to the polarization controller (PC) to adjust the polarization. The collimator adjusts the size of the beam and the polarizer rotates from $0^{\circ}$ to $360^{\circ}$ the $x-y$ polarization. Finally, a CCD camera (Thorlabs BC106-VIS) records the mode patterns. 


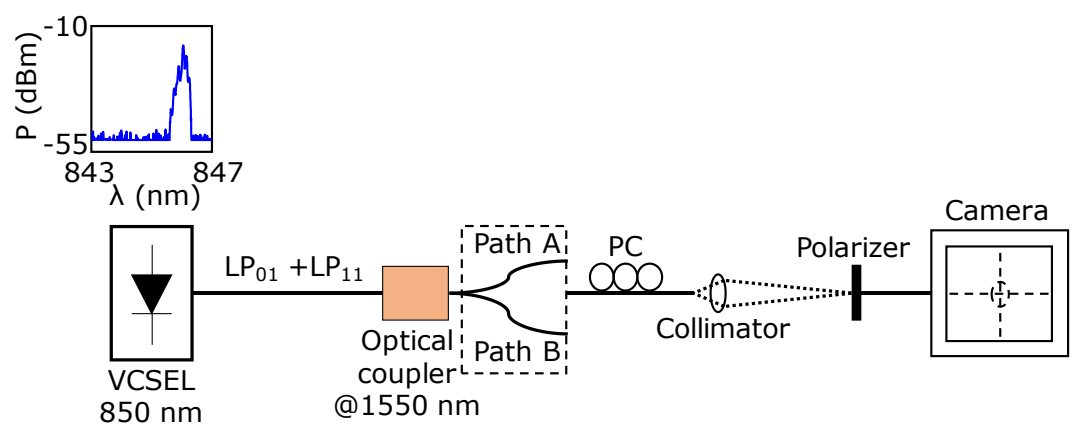

Figure 3.3.5. Experimental setup for the measurement of the near field patterns for the $L P_{01}$ and $L P_{11}$ modes at the direct and cross paths of the fiber coupler.

The experiment is focused on demonstrating the demultiplexing of the $L P_{01}$ and $L P_{11}$ modes, more concretely the $\mathrm{LP}_{11}$ mode coupling. In order to check the coupling performance of the $\mathrm{LP}_{11}$ mode for both sine/cosine azimuthal variation ( $\mathrm{LP}_{11 \mathrm{a}}$ and $\mathrm{LP}_{11 \mathrm{~b}}$ ) the $\mathrm{PC}$ and the polarizer will be jointly adjusted. By rotating 90 degrees the polarizer, it is possible to obtain both orthogonal polarization for any mode.

Different optical couplers with distinct coupling ratios have been measured in order to obtain a complete study of the different $\mathrm{LP}_{01}$ and $\mathrm{LP}_{11}$ modes. The insertion losses and the purity of the optical mode will be measured taking into account the received power and the modal profile of both $L P_{01}$ and $L P_{11}\left(L P_{11 a}-L P_{11 b}\right)$ modes at their respective coupler outputs.

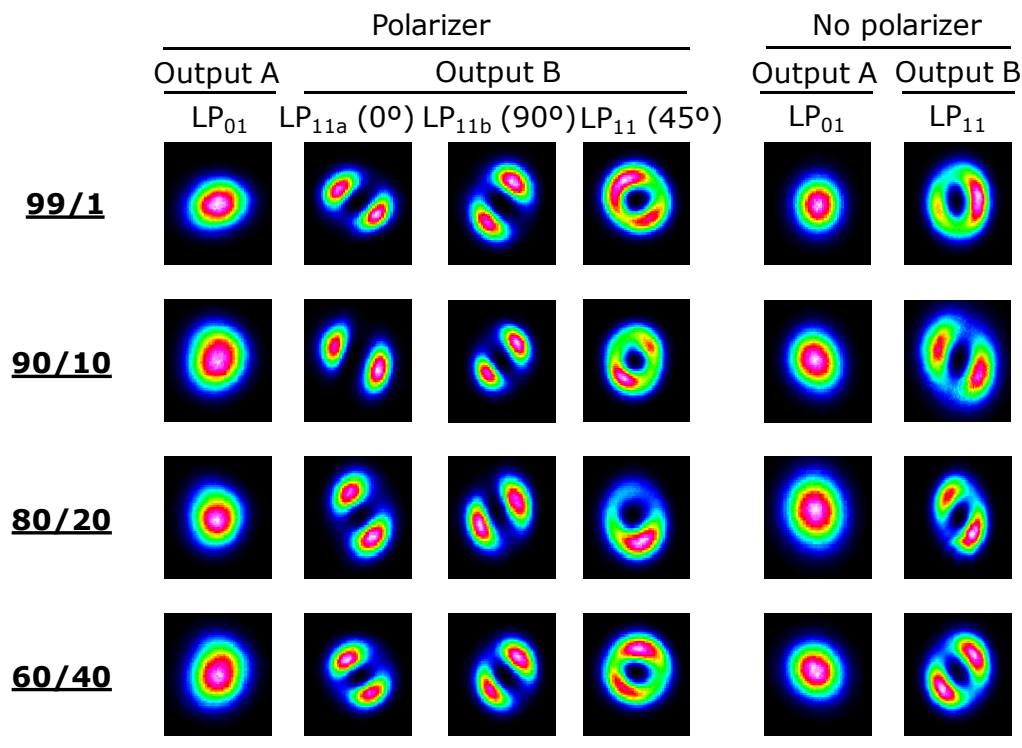

Figure 3.3.6. Experimental near-field pattern at both coupler outputs ( $A$ : direct path, $B$ : cross path) for different optical couplers and pattern rotation (cross-path: 0, 45 and 90 degrees) with/without polarizer in beam path for the $L P_{01}$ mode and $L P_{11 a}$ and $L P_{11 b}$ modes.

Figure 3.3.6 shows the experimental near-field pattern of the $L P_{01}$ and $L P_{11}$ modes at the TMF output. First, when the polarizer is included in the beam path, the $L P_{01}$ mode at the direct path output achieves a perfect near-field pattern for all optical couplers (99/1, 90/10, 80/20 and $60 / 40$ coupling ratios at $1550 \mathrm{~nm}$ ). At the cross path output, a perfect near-field $L P_{11}$ mode profile can be achieved when the polarizer is set at orthogonal linear polarizations, marked as 0 and 90 degrees in Figure 3.3.6 (corresponding in this case to the $\mathrm{LP}_{11 \mathrm{a}}$ and $\mathrm{LP}_{11 \mathrm{~b}}$ orientation), for all couplers considered. However, if the polarizer is 45 degrees rotated, both polarizations are combined and a mixed $\mathrm{LP}_{11 \mathrm{a}} / \mathrm{LP}_{11 \mathrm{~b}}$ mode profile is obtained. At the direct path output, it was observed that a similar $\mathrm{LP}_{01}$ near-field pattern was obtained when changing the polarization. For simplicity, in Figure 3.3.6 only one capture is represented for $\mathrm{LP}_{01}$. 
Table I. Commercial optical couplers at $1550 \mathrm{~nm}$ and $850 \mathrm{~nm}$

\begin{tabular}{cccc}
\hline \multicolumn{2}{c}{ Optical couplers (@1550 nm) } & & Optical couplers (@850 nm) \\
\cline { 1 - 1 } CR $(\%)$ & IL (dB) & & IL (dB) \\
\hline $99 / 1$ & $0.2 / 20.8$ & & $7.4 / 26.2$ \\
$90 / 10$ & $0.7 / 9.2$ & & $4.9 / 12.9$ \\
$80 / 20$ & $1.1 / 7.2$ & & $6.7 / 21.9$ \\
$60 / 40$ & $2.4 / 4.1$ & & $6.9 / 23.8$
\end{tabular}

In terms of the insertion losses, the performance of the $L P_{01}$ and $L P_{11}$ modes at $850 \mathrm{~nm}$ differ from the specifications for the $L P_{01}$ at $1550 \mathrm{~nm}$. Table I summarizes the results. The insertion losses have increased in both branches; being $7.4 \mathrm{~dB}, 4.9 \mathrm{~dB}, 6.7 \mathrm{~dB}$ and $6.9 \mathrm{~dB}$ at the direct path and, $26.2 \mathrm{~dB}, 12.9 \mathrm{~dB}, 21.9 \mathrm{~dB}$ and $23.8 \mathrm{~dB}$ at the cross path, respectively, when the total optical power at the coupler's input is taken as a reference. Thus, the only optical coupler that achieved a similar behavior at $850 \mathrm{~nm}$ than the specifications at $1550 \mathrm{~nm}$ was the 90:10 coupler.

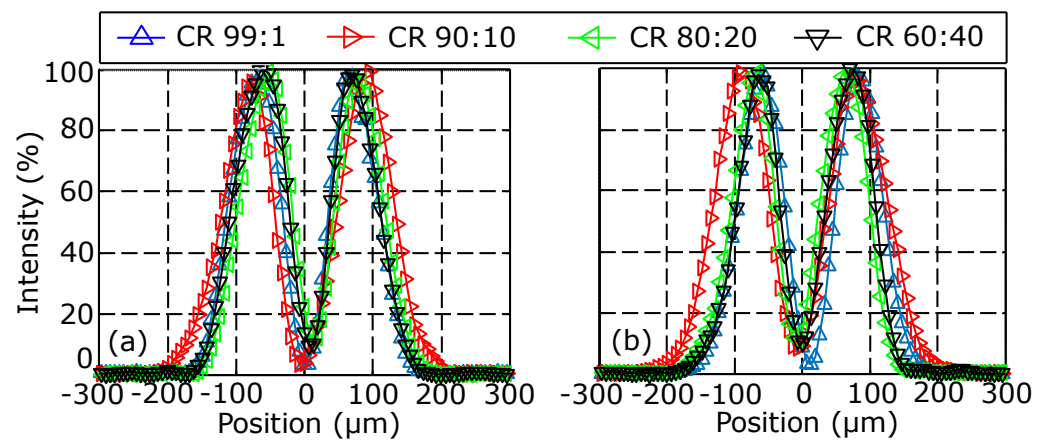

Figure 3.3.7. Experimental modal profiles at the TMF output at $850 \mathrm{~nm}$ for the four optical couplers in the case of the $L P_{11}$ with their two orthogonal polarizations: (a) $L P_{11 a}$ and (b) $L P_{11 b}$

From the $\mathrm{LP}_{11 \mathrm{a}}$ and $\mathrm{LP}_{11 \mathrm{~b}}$ near-field pattern shown in Figure 3.3.6, the corresponding modal profiles along the major axis are shown in Figure 3.3.7. The results of the four optical couplers $(99: 1,90: 10,80: 20$ and $60: 40)$ are compared. Both $L P_{11}$ orientations show an excellent purity after the demultiplexing process. In some cases, there is a minor interference of the $\mathrm{LP}_{01}$ (shown as a non-zero intensity at the center of the fiber, position $=0 \mu \mathrm{m}$ ) due to the use of optical couplers which are designed to operate at $1550 \mathrm{~nm}$ and not optimized for $850 \mathrm{~nm}$ operation. However, if the optical coupler would be specifically designed for $850 \mathrm{~nm}$ operation, the (de)multiplexing of both modes would be perfect [7]. In the $\mathrm{LP}_{11 \mathrm{a}}$ case, the couplers with a coupling ratio of $99: 1$ and $90: 10$ showed in the Figure 3.3.7(a) obtained the best purity with a $97 \%$. The rest of the couplers achieved a $90 \%$. A similar performance is obtained with the $\mathrm{LP}_{11 \mathrm{~b}}$ orientation, but now only the coupler 99:1 (Figure 3.3.7(b)) reached the best purity $(97 \%)$. A purity better than $90 \%$ is obtained for the other three couplers.

\section{CONCLUSIONS}

This paper proposes and demonstrates the simulation and experimental analysis of the $\mathrm{LP}_{01}$ and $\mathrm{LP}_{11}$ modes at $850 \mathrm{~nm}$ when the optical coupler acts as demultiplexer.

The simulation results presents that the 90:10 optical coupler can multiplex/demultiplex the $\mathrm{LP}_{01}$ and $\mathrm{LP}_{11}$ modes at $850 \mathrm{~nm}$ (for a $S=6 \mu \mathrm{m}$ and $L=17 \mathrm{~mm}$ ), obtaining a coupling efficiency of $98 \%$ and $100 \%$, respectively. Moreover, the experimental results point out the good performance of the different optical couplers at $850 \mathrm{~nm}$. In terms of the insertion losses, the 90:10 optical coupler achieved the best performance with insertion losses of $4.9 \mathrm{~dB}$ and $12.9 \mathrm{~dB}$, respectively. The purity of the demultiplexed optical modes was $100 \%$ for the $L P_{01}$ mode and higher than $90 \%$ for both $\mathrm{LP}_{11 \mathrm{a}}$ and $\mathrm{LP}_{11 \mathrm{~b}}$ modes. 
In conclusion, this results open up the possibility to simplify the MDM system using SSMF at $850 \mathrm{~nm}$ without the need of mode converters, mode rotators or mode strippers for mode multiplexing and demultiplexing.

\section{REFERENCES}

[1] P. Winzer, "Making spatial multiplexing a reality," Nat. Photonics 8(5), 345-348 (2014).

[2] D. J. Richardson, J. M. Fini, and L. E. Nelson, "Space-division multiplexing in optical fibres," Nat. Photonics 7(5), 354-362 (2013).

[3] E. Ip, G. Milione, M. J. Li, N. Cvijetic, K. Kanonakis, J. Stone, G. Peng, X. Prieto, C. Montero, V. Moreno, and J. Liñares, "SDM transmission of real-time 10GbE traffic using commercial SFP + transceivers over $0.5 \mathrm{~km}$ elliptical-core few-mode fiber," Opt. Express 23(13), 17120-17126 (2015).

[4] R. G. H. van Uden, R. A. Correa, E. A. Lopez, F. M. Huijskens, C. Xia, G. Li, A. Schülzgen, H. de Waardt, A. M. J. Koonen, and C. M. Okonkwo, "Ultra-high-density spatial division multiplexing with a few-mode multicore fibre," Nat. Photonics 8(11), 865-870 (2014).

[5] T. Mizuno, H. Takara, A. Sano, and Y. Miyamoto, "Dense space division multiplexed transmission overmulti-core andmulti-mode fiber," presented at the Optical Fiber Communication Conf. Exhibition, Los Angeles, CA, USA, Mar. 2015, Paper Th1D.2.

[6] N. Riesen, S. Gross, J. D. Love, Y. Sasaki, and M. J. Withford, "Monolithic mode-selective few-mode multicore fiber multiplexers," Sci. Rep. 7(1), 6971 (2017).

[7] J. L. Corral, D. Garcia-Rodriguez, R. Llorente, "Mode-selective couplers for Two-Mode Transmission at $850 \mathrm{~nm}$ in Standard SMF," Photon. Technol. Lett. 28(4), 425-428 (2016).

[8] A. Li, X. Chen, A. Al Amin, and W. Shieh, "Fused fiber mode couplers for few-mode transmission," IEEE Photon. Technol. Lett. 24(21), 1953-1956 (2012).

[9] R. Ismaeel, T. Lee, B. Oduro, Y. Jung, and G. Brambilla, "All-fiber fused directional coupler for highly efficient spatial mode conversion," Opt. Express 22(10), 11610-11619 (2014).

[10] A. Li, J. Ye, X. Chen, and W. Shieh, "Fabrication of a low-loss fused fiber spatial-mode coupler for few-mode transmission," IEEE Photon. Technol. Lett. 25(20), 1985-1988 (2013).

[11] P. Medina Sevila, V. Almenar and J. L. Corral, "Transmission Over SSMF at $850 \mathrm{~nm}$ : Bimodal Propagation and Equalization," J. Lightwave Technol., 35(19), 4125-4136 (2017).

[12] J. A. Tatum, D. Gazula, L. A. Graham, J. K. Guenter, R. H. Johnson, J. King, C. Kocot, G. D. Landry, I. Lyubomirsky, D. Vaidya, M. Yan, and F. Tang, "VCSEL-based interconnects for current and future data centers," J. Lightwave Technol. 33(4), 727-732 (2015).

[13] S. Moon and D. Y. Kim, "Effective single-mode transmission at wavelengths shorter than the cutoff wavelength of an optical fiber," IEEE Photon. Technol. Lett. 17(12), 2604-2606 (2005).

[14] Z. Tian, C. Chen, and D. V. Plant, "850-nm VCSEL transmission over standard singlemode fiber using fiber mode filter," IEEE Photon. Technol. Lett., 24(5), 368-370 (2012).

[15] D. Garcia-Rodriguez, J. L. Corral, and R. Llorente, "Mode Conversion for Mode Division Multiplexing at $850 \mathrm{~nm}$ in Standard SMF," Photon. Technol. Lett. 29(11), 929-932 (2017).

[16] David Garcia-Rodriguez, Maria Morant, Juan L. Corral and Roberto Llorente, "Mode Multiplexing and Demultiplexing by a Standard Single-Mode Coupler for $850 \mathrm{~nm}$ Few-Mode Transmission Systems", Proc. Eur. Conf. Opt. Commun. (ECOC 2017), Sep. 2017.

[17] D. Marcuse, Theory of dielectric optical waveguides, 2nd ed. Academic, San Diego (1991).

[18] B. Pal, Guided Wave Optical Components and Devices: Basics Technology, and Applications, Elsevier Academic Press, Amsterdam (2005). 


\title{
CHAPTER 4. Mode Division MultipleXing At 1550 NM
}

\subsection{Dimensional variation tolerant mode converter/multiplexer fabricated in SOI technology for two- mode transmission at $1550 \mathrm{~nm}$.}

\section{BIBLIOGRAPHY RECORD}

- Authors: David Garcia-Rodriguez, Juan L. Corral, Amadeu Griol and Roberto Llorente

- Publication: Optics Letters, vol. 42 , no. 7

- Year: 2017

- Pages: 1221-1224

- OCIS codes: (060.1810) Buffers, couplers, routers, switches, and multiplexers; (130.3120) Integrated optics devices; (230.7400) Waveguides, slab; (060.4230) Multiplexing.

\begin{abstract}
The use of an integrated asymmetrical directional coupler for two-mode transmission at $1550 \mathrm{~nm}$ is analysed. The design is based on silicon-on-insulator (SOI) technology and permits mode conversion and mode multiplexing/ demultiplexing. In the nominal design, mode conversion and mode (de)multiplexing are achieved with $97 \%$ efficiency and a $23.4 \mathrm{~dB}$ crosstalk level in the 1540-1560 nm band using a $0.1 \mu \mathrm{m}$ gap. The dimension tolerance of the SOI process has been taken into account in the selection of the optimum design, and the coupling efficiency would remain above $82.3 \%$ (corresponding to $0.8 \mathrm{~dB}$ excess loss) with $3 \sigma$ accuracy. A $90 \%$ efficiency has been experimentally obtained.
\end{abstract}


Recently, significant research efforts have focused on increasing the optical fiber transmission capacity to cope with the rising demand for information transmission. The standard single-mode fiber (SSMF) worldwide deployed operates exclusively in the infra-red band (1.3$1.6 \mu \mathrm{m})$. Although the exploitable capacity in the infra-red band is substantial, it shows signs of exhaustion, even when modern modulation schemes are used [1].

Mode division multiplexing (MDM) is a good solution to overcome the limit on the fiber capacity. New devices such as mode (de)multiplexers to combine and split the modes [2-5] would be required. Different techniques have been proposed to convert and (de)multiplex the modes: for example, liquid crystal on silicon, directional couplers (DCs), asymmetrical directional couplers (ADCs), and long-period fiber Bragg gratings.

ADCs are a compact solution that provide mode conversion and mode (de)multiplexing by exciting the higher order mode from the fundamental one.

Mode conversion is induced by matching the effective index of the higher mode in the wide fiber/waveguide and the effective index of the fundamental mode in the narrow fiber/waveguide [2-11]. ADCs are low loss and cheap devices. The fabrication of ADCs in integrated technology offers lower size, better repeatability, and higher robustness than the fiber-based devices.

Silica-based planar lightwave circuit (PLC) technology has been used to fabricate ADC [5-8], but higher sizes and a higher bending radius (above $5 \mathrm{~cm} \mathrm{[6])} \mathrm{are} \mathrm{required.} \mathrm{On} \mathrm{the} \mathrm{other} \mathrm{side,}$ silicon-on-insulator (SOI) technology offers a reduced size and a lower bending radius, which clearly improve the option to integrate several functions on the same chip $[9,10]$. However, the performance of SOI-based ADCs recently proposed are quite sensitive to dimensional variations, and excess losses around $2 \mathrm{~dB}$ or crosstalk levels up to $10 \mathrm{~dB}$ should be accepted for waveguide width variations of $\Delta w= \pm 5 \mathrm{~nm} \sim \pm 10 \mathrm{~nm}$ [9]. Unfortunately, size deviations up to $\pm 10 \mathrm{~nm}$ are not uncommon in typical SOI technology [12], which would lead to low device yields or high thermal tuning powers.

In this Letter, a comprehensive study of the optimum dimensions for an ADC mode converter and (de)multiplexer in SOI technology is presented. Both the nominal performance of the ADC at the design wavelength and around that wavelength are considered, and the best configuration is selected in terms of the sensitivity to the dimensional variations due to the fabrication accuracy of the state-of-the-art SOI processes. The selected device has been fabricated and measured.

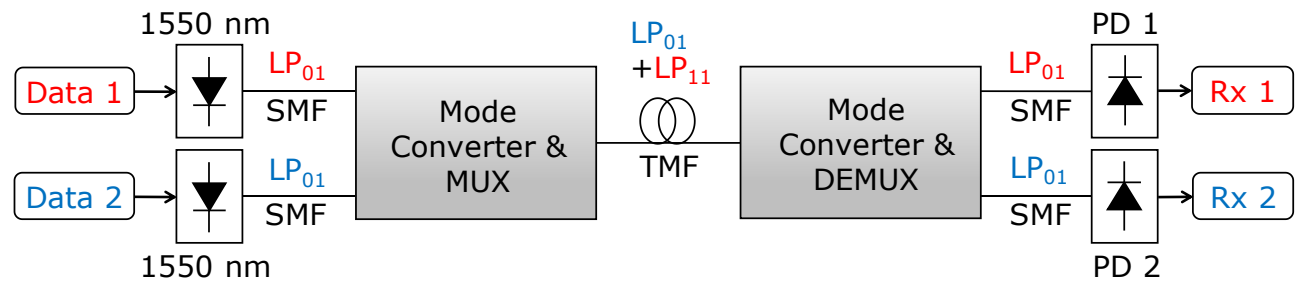

Figure 4.1.1. Scheme for mode division multiplexing (MDM) at $1550 \mathrm{~nm}$ with two-mode fiber (TMF) optical transmission media.

The general concept of the MDM link is depicted in Figure 4.1.1. The system consists of two lasers emitting at $1550 \mathrm{~nm}$ propagating the $L P_{01}$ mode in a single-mode fiber (SMF). The two $\mathrm{LP}_{01}$ modes are coupled to the SOI mode converter through grating couplers, where they are converted to $\mathrm{TE}_{0}$ and $\mathrm{TE}_{1}$ modes and multiplexed to the common output. Both optical signals are then coupled to the two-mode fiber (TMF), corresponding now to the $L P_{01}$ and $L P_{11}$ modes. At the receiver side, the same $A D C$ will be needed to demultiplex each mode to the corresponding photodiode. 

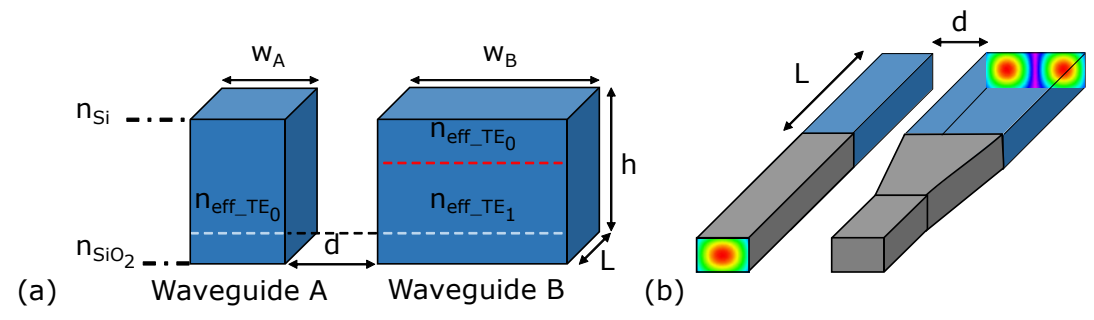

Figure 4.1.2. (a) Refractive index profile for mode coupling between $T E_{0}$ and $T E_{1}$ modes and (b) a $3 D$ sketch of the mode converter.

The mode converter (Figure 4.1.2) is constructed as an asymmetrical coupler, where the waveguide widths are not equal. The operation principle is regulated by the phase matching condition, where the effective index of the $\mathrm{TE}_{0}$ mode in the waveguide $\mathrm{A}\left(w_{A}\right)$ must match the effective index of the $\mathrm{TE}_{1}$ mode in the waveguide $\mathrm{B}\left(w_{B}\right)$. The waveguides in the $\mathrm{ADC}$ are separated a distance, $d$, with a certain coupling length, $L$, and height, $h$.

According to the coupled-mode theory, the optical fields along each waveguide can be expressed as

$$
\begin{aligned}
& \vec{E}^{A}(x, y, z, t)=A(z) \vec{\varepsilon}_{m n}^{A}(x, y) e^{j\left(\beta_{m n}^{A} z-\omega t\right)} \\
& \vec{E}^{B}(x, y, z, t)=B(z) \vec{\varepsilon}_{p q}^{B}(x, y) e^{j\left(\beta_{p q}^{B} z-\omega t\right)}
\end{aligned}
$$

where $\vec{\varepsilon}_{m n}^{A}(x, y)$ and $\vec{\varepsilon}_{p q}^{B}(x, y)$ are the modal profiles of the modes under consideration in each waveguide ( $m n$ in the waveguide $\mathrm{A}$ and $p q$ in the waveguide $\mathrm{B}$ ), while $\beta_{m n}^{A}$ and $\beta_{p q}^{A}$ are the propagation constants for both modes, respectively. The modal profiles and propagation constants correspond to the isolated waveguide modes, whereas the influence of the local modal profiles will be considered in the variation of the complex envelopes, $A(z)$ and $B(z)$, along the direction of propagation.

Assuming $L$ is the length of the directional coupler, if only one mode is present at the waveguide A input of the coupler $(B(0)=0)$, the optical power in both outputs (direct $(A)$ and coupled $(B)$ paths) as a function of the input power $P_{a}(0)$ is [13]

$$
\begin{gathered}
\frac{P_{a}(L)}{P_{a}(0)}=\left|\frac{A(L)}{A(0)}\right|^{2}=\left(\frac{\kappa_{a b} \kappa_{b a}}{\beta_{c}^{2}}\right) \cos ^{2}\left(\beta_{c} L\right)+\left(\frac{\delta}{\beta_{c}}\right)^{2} \\
\frac{P_{b}(L)}{P_{a}(0)}=\left|\frac{B(L)}{A(0)}\right|^{2}=\frac{\left|\kappa_{b a}\right|^{2}}{\beta_{c}^{2}} \sin ^{2}\left(\beta_{c} L\right) \\
\delta=\frac{\left(\beta_{p q}^{B}+\kappa_{b b}\right)-\left(\beta_{m n}^{A}+\kappa_{a a}\right)}{2} \\
\beta_{c}=\sqrt{\kappa_{a b} \kappa_{b a}+\delta^{2}}
\end{gathered}
$$

where $\kappa_{a a}$ and $\kappa_{b b}$ are the self-coupling coefficients that take into account the variation in the propagation constant of each mode due to the perturbation caused by the other waveguide; $\kappa_{a b}$ and $\kappa_{b a}$ are the mutual coupling coefficients of the waveguide B to A and vice versa which depend on the fiber separation, $d$; and $\delta$ is the phase mismatch. Finally, the coupling efficiency is defined as

$$
\eta=\frac{P_{b}(L)}{P_{a}(0)}=\frac{\left|\kappa_{b a}\right|^{2}}{\beta_{c}^{2}} \sin ^{2}\left(\beta_{c} L\right)
$$

Therefore, the optical power is periodically exchanged between both waveguides appearing at the first maximum in the coupled output for a distance: 


$$
L_{c}=\frac{\pi}{2 \beta_{c}}
$$

The power transfer will be maximized when the phase-matching condition is fulfilled $(\delta=0)$, which implies that the propagation constants of both modes in the coupler have to be the same and, therefore, the refractive indices will also be equal.

When the coupler is made of similar waveguides (DC), the phase match condition is always fulfilled if the modes to be coupled are the same one $(A=B)$, regardless of the wavelength. However, in an ADC, the effective indices of the modes to be coupled, which are wavelength dependent, must be matched by properly selecting the dimensions of the respective waveguides to be used.

A silicon-on-insulator wafer with a silicon thickness of $220 \mathrm{~nm}$ has been selected for the design of the coupler, as it has become the standard substrate for several established research and development foundries [12]. The refractive indices of $\mathrm{Si}$ and $\mathrm{SiO}_{2}$ are $n_{\mathrm{Si}}=3.47$ and $n_{\mathrm{SiO}_{2}}=1.46$, respectively, and the eigenmodes' effective indices in the waveguides will be calculated with the three-dimensional (3D) beam propagation method (3D-BPM). For this height, the TE polarization has lower bending losses [12].

$A D C$ is made of a single-mode waveguide propagating the $\mathrm{TE}_{0}$ mode and a two-mode waveguide propagating both $\mathrm{TE}_{0}$ and $\mathrm{TE}_{1}$ modes. $\mathrm{TE}_{0}$ enters the single-mode waveguide, and it is converted to $\mathrm{TE}_{1}$ in the two-mode waveguide. The width of the single-mode waveguide is taken as $w_{A}=0.45 \mu \mathrm{m}$ with an effective index value of $n_{\text {eff_TEO }}=2.4109$ at $1550 \mathrm{~nm}$. The effective index of $\mathrm{TE}_{0}$ in the single-mode waveguide must match the $\mathrm{TE}_{1}$ in the two-mode waveguide which can be accomplished by increasing the width of the two-mode waveguide up to $w_{B}=0.962 \mu \mathrm{m}$. For this width, the effective index values are $n_{\text {eff }}$ TEO $=2.7390$ and $n_{\text {eff_TE1 }}=2.4106$.

$T E_{0}$ to $T E_{1}$ coupling efficiency and $T E_{0}$ insertion losses are the most important parameters to evaluate the performance of the ADC as mode converter and multiplexer. Due to the effective index difference, both $\mathrm{TE}_{0}$ modes do not interact, and the $\mathrm{TE}_{0}$ insertion losses in the two-mode waveguide are negligible.

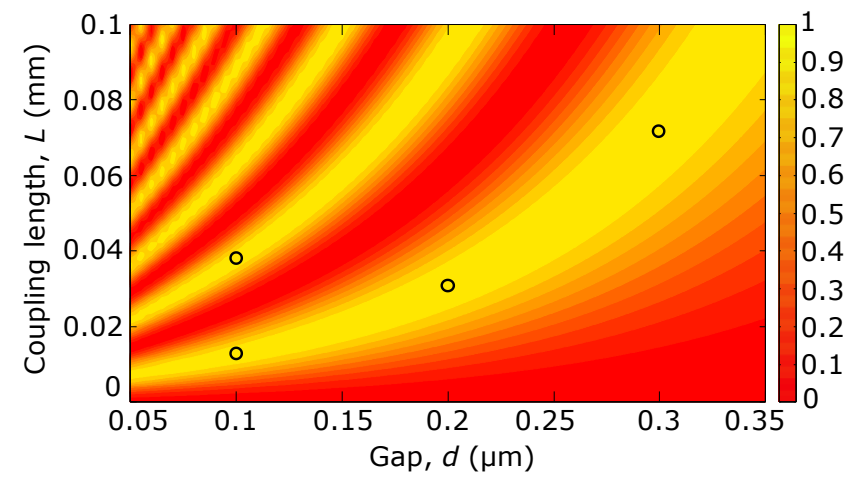

Figure 4.1.3. Coupling efficiency from $T E_{0}$ to $T E_{1}$ for an asymmetrical directional coupler with the refractive index profile from Figure 4.1.2.

The ADC has been simulated at $1550 \mathrm{~nm}$ for different gap, $d(\mu \mathrm{m})$, and coupling length, $L(\mathrm{~mm})$, values. The coupling efficiency results are depicted in Figure 4.1.3, where it is shown that for any given gap value the maximum coupling efficiency is achieved for different coupling lengths, according to equation (4.1.7).

For wider gaps between the waveguides, the mutual coupling coefficients are reduced and, therefore, the ADC requires longer coupling lengths to achieve the maximum coupling efficiency.

Different gap/length combinations have been selected in order to compare the performance of the $A D C$ around $1550 \mathrm{~nm}$ and its robustness against fabrication tolerances. The four cases are 
highlighted in Figure 4.1.3 and correspond to these dimensions: ( $\left.d=0.1 \mu \mathrm{m}, L_{1}=12.62 \mu \mathrm{m}\right)$, $\left(d=0.1 \mu \mathrm{m}, L_{2}=38.10 \mu \mathrm{m}\right),(d=0.2 \mu \mathrm{m}, L=30.98 \mu \mathrm{m})$, and $(d=0.3 \mu \mathrm{m}, L=72.08 \mu \mathrm{m})$.

When the $A D C$ acts as a mode demultiplexer, both $T E_{0}$ and $T_{1}$ modes enter the two-mode waveguide, and each mode should exit the coupler from different outputs. ( $\mathrm{TE}_{0}$ remains in the two-mode waveguide, whereas the $\mathrm{TE}_{1}$ is converted to $\mathrm{TE}_{0}$ in the single-mode output.) In addition to the previously considered parameters, the crosstalk between both outputs is the most important parameter to assess; namely, the extinction ratio of the $\mathrm{TE}_{1}$ mode in the two-mode waveguide and the undesired leakage of the $\mathrm{TE}_{0}$ mode to the single-mode waveguide.

(a)
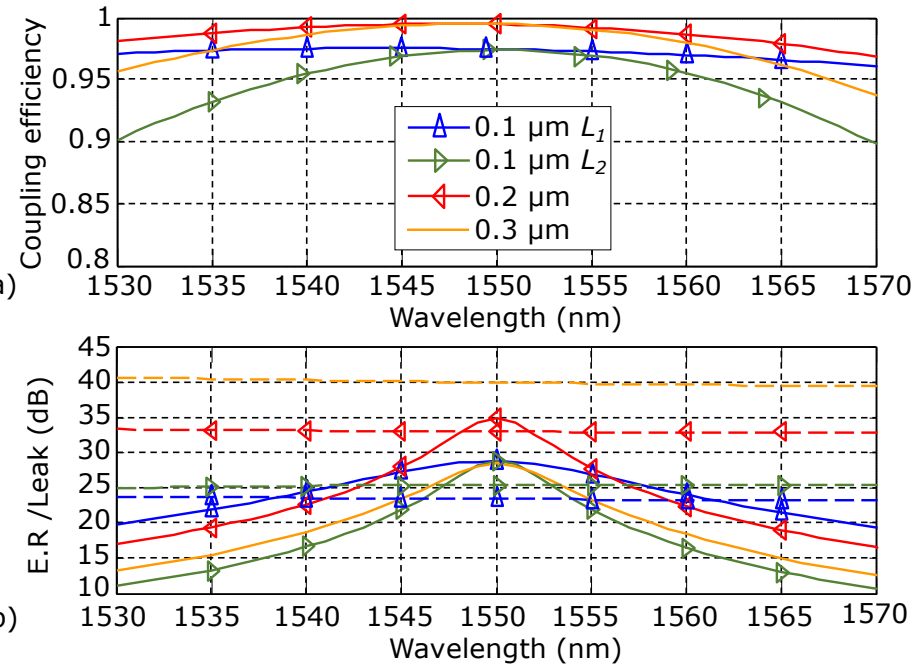

Figure 4.1.4. Coupling efficiency from $T E_{0}$ to $T E_{1}$ and (b) $T E_{1}$ extinction ratio and $T E_{0}$ leakage (dashed line) for the asymmetrical directional coupler from Figure 4.1.2 for different combinations of gap, $d$, and coupler length, $L$.

If the performance of all ADC parameters is considered both at $1550 \mathrm{~nm}$ and its evolution around the design wavelength, the optimum dimensions of all four selected cases are slightly modified as $\left(d=0.1 \mu \mathrm{m}, L_{1}=12.50 \mu \mathrm{m}\right),\left(d=0.1 \mu \mathrm{m}, L_{2}=37.74 \mu \mathrm{m}\right),(d=0.2 \mu \mathrm{m}, L=30.74 \mu \mathrm{m})$, and $(d=0.3 \mu \mathrm{m}, L=70.92 \mu \mathrm{m})$. The coupling efficiency associated with these four cases is depicted in Figure 4.1.4(a), where the wavelength dependence of the effective modal indices is also considered in the simulations. These results show that all cases offer an efficiency better than $97.4 \%$ at $1550 \mathrm{~nm}$. If a $20 \mathrm{~nm}$ bandwidth around the design wavelength is considered, the best performance in terms of coupling efficiency is obtained for a $0.2 \mu \mathrm{m}$ gap $(L=30.74 \mu \mathrm{m})$ with a $98.7 \%$ coupling efficiency (corresponding to $0.06 \mathrm{~dB}$ excess loss), compared to $97 \%$ for a $0.1 \mu \mathrm{m}$ gap. From the results in Figure 4.1.4(a), it can be stated that for any given gap distance the shortest length offering the maximum coupling should be selected, as the $\left(d=0.1 \mu \mathrm{m}, L_{2}=37.74 \mu \mathrm{m}\right)$ case is quite a bit more wavelength sensitive than the $(d=0.1 \mu \mathrm{m}$, $L_{1}=12.50 \mu \mathrm{m}$ ) case. For all three cases, with the shortest length, the coupling efficiency in $C$ band is better than $95.5 \%$ or $0.2 \mathrm{~dB}$ excess loss.

In Figure 4.1.4(b), it can be seen that all four cases have $\mathrm{TE}_{1}$ extinction ratio levels better than $28.4 \mathrm{~dB}$ at $1550 \mathrm{~nm}$ and as high as $34.7 \mathrm{~dB}$ for the best configuration. However, the $\mathrm{TE}_{1}$ extinction ratio is quite sensitive to wavelength variation, and the best performance is obtained for the $\left(d=0.1 \mu \mathrm{m}, L_{1}=12.50 \mu \mathrm{m}\right)$ case where an extinction ratio better than $24.2 \mathrm{~dB}$ is achieved in a $20 \mathrm{~nm}$ range around $1550 \mathrm{~nm}$. From this figure, it can be stated that the wider the gap between both waveguides, the lower the $\mathrm{TE}_{1}$ extinction ratio in a certain bandwidth around the design wavelength.

In terms of the $\mathrm{TE}_{0}$ leakage, the results from Figure 4.1.4(b) show that the lowest leakage is obtained when the waveguides are further separated, as could be expected due to the highest confinement of this mode. The $\mathrm{TE}_{0}$ leakage dependence on the wavelength is quite low, and a level better than $23.4 \mathrm{~dB}$ is obtained on a $20 \mathrm{~nm}$ bandwidth. All in all, the best performance in 
terms of crosstalk is obtained for the $\left(d=0.1 \mu \mathrm{m}, L_{1}=12.50 \mu \mathrm{m}\right)$ case. A narrower gap would impair further the $\mathrm{TE}_{0}$ crosstalk level. If the results for both cases with a $d=0.1 \mu \mathrm{m}$ gap are compared, the case with a shortest length offers again the best results.

In terms of the nominal design, all four cases offer an outstanding performance as mode converter and mode multiplexer/demultiplexer at the design wavelength. When a certain bandwidth is considered, the shortest coupling length for any given gap separation is always preferred. In these cases, there are some minor differences between the three gap values analyzed, and the selection of the best design will depend on which parameter (coupling efficiency, $\mathrm{TE}_{1}$ extinction ratio or $\mathrm{TE}_{0}$ leakage) is considered to be the most critical for the application.

We will now take into account the tolerances of the fabrication process in terms of waveguide width variations. We will consider a SOI CMOS-compatible process based on a $193 \mathrm{~nm}$ optical deep UV dry lithography and dry etching for $200 \mathrm{~mm}$ wafers as a typical process to be found in fabrication foundries. The $3 \sigma$ accuracy of the linewidth uniformity for this process has been found to be $\pm 8 \mathrm{~nm}$ [11]. Thus, the waveguide dimensions of the ADCs can be estimated to fulfill these accuracies with a $99.7 \%$ probability.

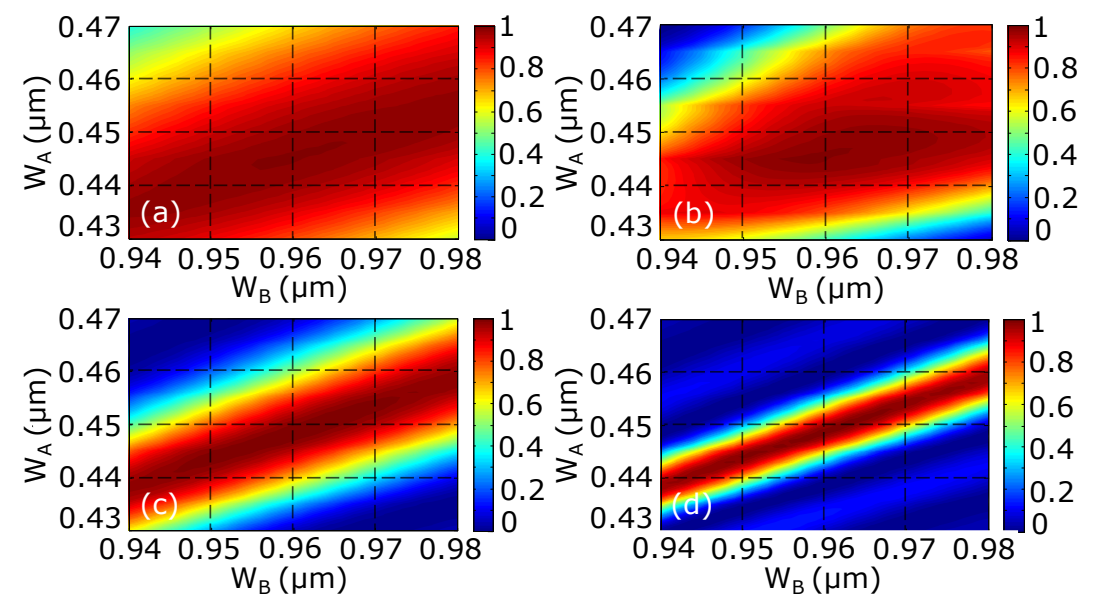

Figure 4.1.5. Sensitivity of $T E_{0}$ to $T E_{1}$ coupling efficiency to the variation of the waveguide widths.

(a) $0.1 \mu \mathrm{m} L_{1}$, (b) $0.1 \mu \mathrm{m} \mathrm{L} L_{2}$ (c) $0.2 \mu \mathrm{m}$, and (d) $0.3 \mu \mathrm{m}$.

The ADC configurations previously selected have been simulated for $w_{A}$ and $w_{B}$ waveguide widths, ranging from 0.43 to $0.47 \mu \mathrm{m}$ and 0.94 to $0.98 \mu \mathrm{m}$, respectively. The sensitivity of the coupling efficiency to these width variations is shown in Figure 4.1.5 for all four gap/length combinations previously selected. It can be seen that the best configuration in terms of coupling efficiency $(0.2 \mu \mathrm{m}$ gap), according to the nominal widths, offers a less robust design than the configurations with a narrower gap, $d=0.1 \mu \mathrm{m}$. Using a $0.1 \mu \mathrm{m}$ gap instead of the $0.2 \mu \mathrm{m}$ one implies that a shorter coupling length is needed. Therefore, any changes in the waveguide dimensions and, consequently, in the propagation constants, will affect to a lesser extent the coupling efficiency when equation (4.1.7) is considered.

In fact, Figure 4.1.5(a) shows a region where the efficiency coupling remains quite high if the variation in the widths is within $\pm 8 \mathrm{~nm}$, obtaining an efficiency above $82.3 \%$ or $0.8 \mathrm{~dB}$ excess loss.

However, when the gap between waveguides is 0.2 and $0.3 \mu \mathrm{m}$, the same width variation would imply quite a reduction in the coupling efficiency, obtaining minimum values around $46 \%$ and $0 \%$, respectively. An interesting result from Figure 4.1 .5 is that the coupling efficiency is at its maximum and remains constant when the width variations are proportional in both waveguides. If the variation of the coupling efficiency along any of the axes is compared, it can be seen that the accuracy of the width of the single-mode waveguide is more critical than the variation of the width for the wide waveguide. 
In order to test the thermal sensitivity of the $A D C$, the silicon thermo-optic coefficient $\left(1.86 \cdot 10^{-4} \mathrm{~K}^{-1}\right)$ has been considered in the simulations, and a coupling efficiency variation below $0.2 \%$ has been obtained when a $\pm 20 \mathrm{~K}$ temperature range is considered.

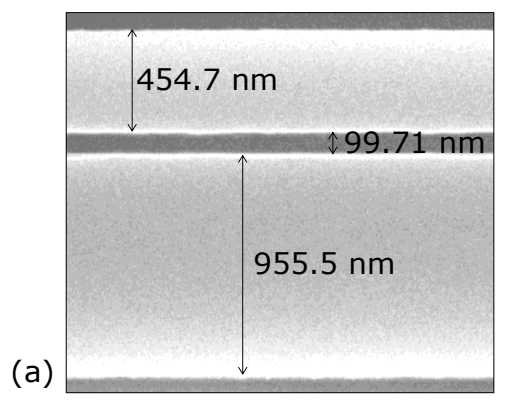

(b)

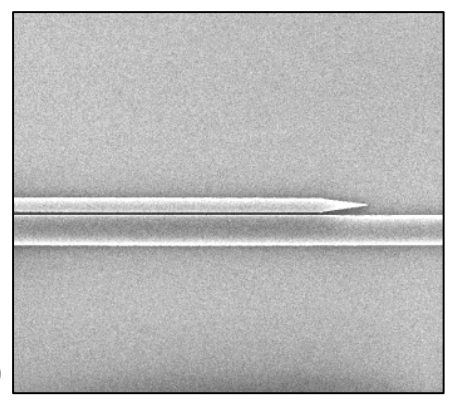

Figure 4.1.6. SEM image. (a) $w_{A}, w_{B}$ and gap values and (b) coupling zone.

The ADC with a $0.1 \mu \mathrm{m}$ gap has been fabricated in the NTC laboratory with a SOI CMOS-compatible process based on an E-beam lithography [14]. The device is made of two independent single-mode waveguides (width $w_{A}$ ) coupled through an intermediate two-mode waveguide (width $w_{B}$ ) so a double $T E_{0}-T E_{1}$ and $T E_{1}-T E_{0}$ coupling would take place. Both input and output single-mode waveguides are vertically coupled to an SSMF fiber by means of grating couplers. In order to avoid any undesirable reflection, the waveguides were tapered after the coupling zone, as shown in Figure 4.1.6(b), and the coupling length (straight zone) was reduced to $L=12.30 \mu \mathrm{m}$ to take into account the additional coupling within the tapered waveguides. The measurement results for this device show that the experimental coupling efficiency has a similar response to the theoretical one at the $1530-1570 \mathrm{~nm}$ band. The experimental coupling efficiency is $90 \%$ at $1550 \mathrm{~nm}, 7.5 \%$ below the theoretical coupling, corresponding to an additional $0.35 \mathrm{~dB}$ excess loss. If a $20 \mathrm{~nm}$ bandwidth around the design wavelength is considered, the experimental coupling is higher than the $84 \%$, as is shown in Figure 4.1.7. The small differences between theoretical and experimental coupling are due to waveguide and gap variations in the fabrication process, as can be seen in Figure 4.1.6(a).

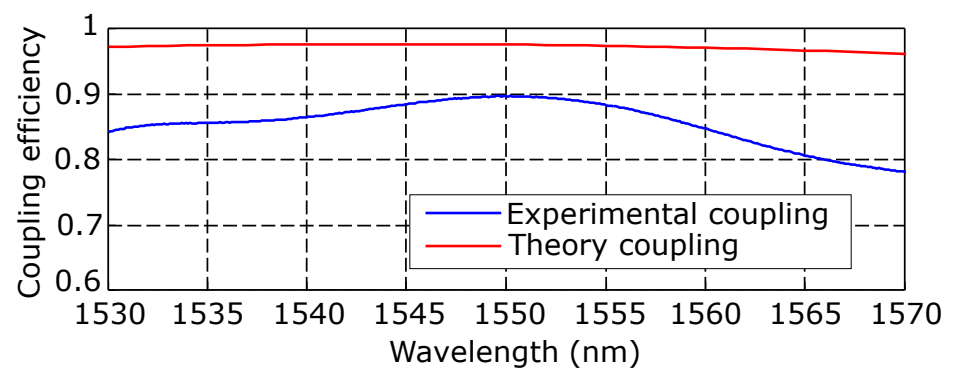

Figure 4.1.7. Theoretical and experimental conversion efficiency from the $T E_{0}$ to $T E_{1}$ mode for a $0.1 \mu \mathrm{m}$ gap.

Figure 4.1.6 shows a SEM image of the fabricated sample. In Figure 4.1.6(a), which is a zoomed area from Figure 4.1.6(b), it is possible to see the actual dimensions of the waveguide widths and the coupling gap in the coupling zone. According to Figure 4.1.5(a), the achieved dimension variations shown in Figure 4.1.6(a) will correspond to an efficiency of $90 \%$ at $1550 \mathrm{~nm}$, similar to the measured value. If the same error had occurred in the 0.2 and $0.3 \mu \mathrm{m}$ gap devices, the efficiency would be around $60 \%$ and $20 \%$, respectively.

\section{REFERENCES}

[1] J. J. Benson, Jr., S. Spaelte, and M. V. Shastri, in Optical Fiber Communication Conference and National Fiber Optic Engineers Conference, Anaheim, California, USA, 2013, paper OTh4B.7. 
[2] T. Uematsu, K. Saitoh, N. Hanzawa, T. Sakamoto, T. Matsui, K. Tsujikawa, and M. Koshiba, in Optical Fiber Communication Conference and National Fiber Optic Engineers Conference, Anaheim, California, USA, 2013, paper OTh1B.5.

[3] M. Greenberg and M. Orenstein, Opt. Express 13, 9381 (2005).

[4] T. Uematsu, Y. Ishizaka, Y. Kawaguchi, K. Saitoh, and M. Koshiba, J. Lightwave Technol. 30, 2421 (2012).

[5] Y. H. Ding, J. Xu, F. D. Ros, B. Huang, H. Y. Ou, and C. Peucheret, Opt. Express 21, 10376 (2013).

[6] N. Hanzawa, K. Saitoh, T. Sakamoto, T. Matsui, K. Tsujikawa, M. Koshiba, and F. Yamamoto, Opt. Express 22, 29321 (2014).

[7] N. Hanzawa, K. Saitoh, T. Sakamoto, T. Matsui, K. Tsujikawa, M. Koshiba, and F. Yamamoto, Opt. Express 21, 25752 (2013).

[8] N. Hanzawa, K. Saitoh, T. Sakamoto, T. Matsui, K. Tsujikawa, T. Uematsu, and F. Yamamoto, J. Lightwave Technol. 33, 1161 (2015).

[9] J. Wang, S. He, and D. Dai, Laser Photon. Rev. 8, L18 (2014).

[10] D. Dai, J. Wang, and Y. Shi, Opt. Lett. 38, 1422 (2013).

[11] J. L. Corral, D. Garcia-Rodriguez, and R. Llorente, IEEE Photon. Technol. Lett. 28, 425 (2016).

[12] D. X. Xu, J. H. Schmid, G. T. Reed, G. Z. Mashanovich, D. J. Thomson, M. Nedeljkovic, X. Chen, D. Van Thourhout, S. Keyvaninia, and S. K. Selvaraja, IEEE J. Sel. Top. Quantum Electron. 20, 422 (2014).

[13] J. M. Liu, Photonic Devices (Cambridge University, 2007).

[14] A. E. Grigorescu, M. C. van der Krogt, and C. W. Hagen, Proc. SPIE 6519, 65194A (2007). 


\subsection{Design of Asymmetrical Directional Couplers on Ridge and Strip SOI technology with high dimensional variation tolerance}

\section{BIBLIOGRAPHY RECORD}

- Authors: David Garcia-Rodriguez, Juan L. Corral and Roberto Llorente

- Publication: Optics Letters, vol. 43, no. 11

- Year: 2018

- Pages: 2491-2494

- OCIS codes: (060.1810) Buffers, couplers, routers, switches, and multiplexers; (060.4230) Multiplexing; (130.3120) Integrated optics devices; (230.7400) Waveguides, slab.

\section{ABSTRACT}

The supermode analysis of asymmetrical directional couplers (ADC) based on SOI technology for strip and ridge structures at $1550 \mathrm{~nm}$ is herein reported targeting to reduce ADC device fabrication requirements. The reported analysis based on supermodes permits to assess the sensitivity of the ADC coupling efficiency by calculating the index difference between even and odd supermodes. Optimum designs have been found for $100 \mathrm{~nm}$ and $400 \mathrm{~nm}$ gaps, respectively, capable of converting and (de) multiplexing both $\mathrm{TE}_{0}$ and $\mathrm{TE}_{1}$ modes taking into account the width, gap, height and slab thickness variations produced with respect to the nominal design. 
Data traffic demand is nowadays increasing dramatically due to the introduction of new telecommunication services like machine-to-machine communications in the internet-of-things (IoT) paradigm, and the continuous increase of video consumption in high bitrate-demanding formats as 4K [1]. Optical fiber capacity is increasing at approximately one order of magnitude per year [2]. If current growth rate continues, the commercial capacity may reach its upper limit within the next decade. In order to accommodate future data traffic growth, the space-dimension must be considered in the data multiplexing scheme [3].

Spatial Division multiplexing (SDM), both in multicore fiber [4], or in few mode fibers supporting mode division multiplexing (MDM), has been appointed as promising technique to avoid this capacity crunch [5-15]. Silicon on insulator (SOI) or planar lightwave circuit (PLC) processing technologies are optimal candidates to produce cost effective optical devices for modal multiplexing in MDM transmission systems [8-17]. In such systems, mode (de)multiplexers are the key devices. Several mode (de)multiplexers based on Asymmetric Directional Couplers (ADCs) [8], Y-junction [10] or in multimode interference (MMI) [13] have been reported in the literature.

These devices are typically fabricated in PLC technology, which offers several advantages such as low insertion loss, high yield rate and mass productivity; especially as the mode number increases [11,12]. However, higher sizes and long bending radii are usually required (exceeding $5 \mathrm{~cm}$ in some cases [14]). However, SOI technology offers a reduced size and shorter bending radii, which clearly improve the capability to integrate several functions [15].

ADC devices based on SOI technology with strip structure offer an excellent performance as mode (de)multiplexer but they require a precise phase matching of the different optical modes and therefore they are inherently sensitive to fabrication errors $[8,9,15]$. In order to ease tolerances in the fabrication process, the analysis of the effective refractive indexes of the even and odd supermodes have been recently used to study the performance and the structure of different integrated devices based on coupling waveguides [16,17]. This method has been used to analyze the tolerance against variations in width, height and gap for strip and ridge directional couplers (DC), demonstrating that the DC ridge design is 4 times more robust [17].

This letter address the design of an ADC in SOI technology to convert between $\mathrm{TE}_{0}$ and $\mathrm{TE}_{1}$ modes with the objective of maximizing the tolerance against dimensional variations due to the fabrication process. The ADC coupling efficiency is expressed in terms of the effective index difference between even and odd supermodes and an analysis of the sensitivity of the coupling efficiency to variations on the width, height, gap or etching depth dimensions has been carried out for both strip and ridge ADCs. Design guidelines to maximize the device tolerance are given and the best design is selected.

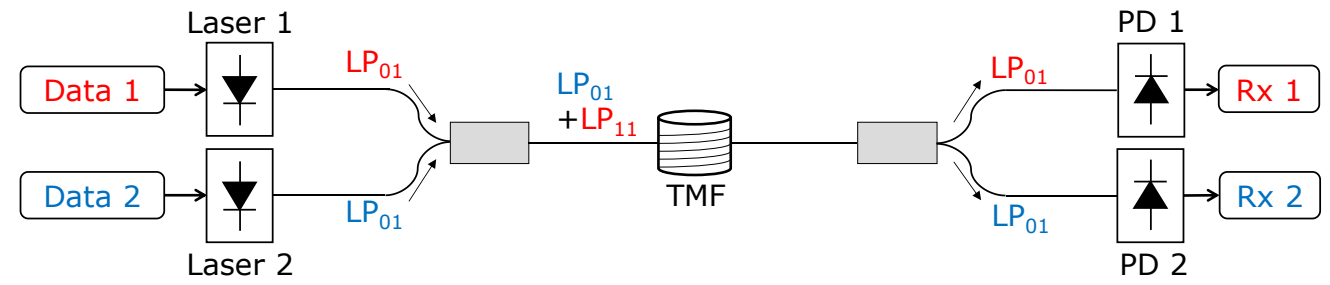

Figure 4.2.1. Scheme for mode division multiplexing (MDM) at $1550 \mathrm{~nm}$ with two mode fiber (TMF) optical transmission media.

Figure 4.2.1 depicts the scheme of a MDM link. At the transmitter, two directly modulated lasers emit at $1550 \mathrm{~nm}$ propagating the $\mathrm{LP}_{01}$ modes in their respective single-mode fibers (SMFs). One $\mathrm{LP}_{01}$ mode must be converted to a higher mode ( $L \mathrm{P}_{11}$ ) and multiplexed with the other $\mathrm{LP}_{01}$ mode. In our case, an ADC based on SOI technology has been selected as mode converter and multiplexer. Both $\mathrm{LP}_{01}$ modes will be coupled to the SOI ADC through input grating couplers converting the signals to $\mathrm{TE}_{0}$ modes. One of the $\mathrm{TE}_{0}$ modes (corresponding to the upper branch in Figure 4.2.1) will be converted to the $\mathrm{TE}_{1}$ and multiplexed to a common waveguide. Finally, both modes will be coupled to the two-mode fiber (TMF) through an output 
grating coupler, propagating now the $\mathrm{LP}_{01}$ and $\mathrm{LP}_{11}$ modes. At the receiver side, the same ADC is used to demultiplex each mode to the corresponding photodiode.

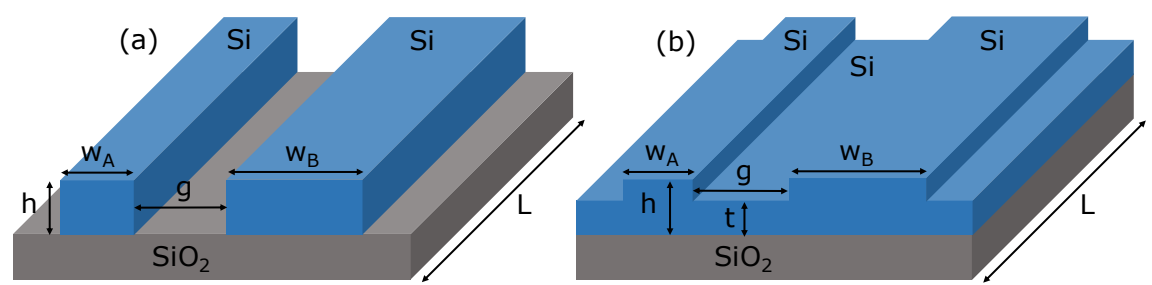

Figure 4.2.2. Mode converter and (de)multiplexer based on SOI technology for two structure types. (a) Strip and (b) ridge.

We propose two different designs, strip and ridge, for the ADC, as is depicted in Figure 4.2.2. In both cases, the waveguide widths are not equal and the device operation depends on achieving the phase matching condition, where the effective index of the $T_{E_{0}}$ mode in the waveguide $A\left(w_{A}\right)$ must match the effective index of the $T_{1}$ mode in the waveguide $B\left(w_{B}\right)$. Besides the waveguide widths, the main parameters used in the device optimization are the gap between waveguides, $g$, the coupling length, $L$, the height, $h$, and the slab thickness, $t$ ( $t=0$ for the strip structure).

Considering that the power is launched only into mode $a$ at $z=0$ (initial conditions $A(0) \neq 0$ and $B(0)=0)$, the mode expansion coefficients in the two coupled waveguides $(A(z)$ mode $a$ in waveguide $A$ and $B(z)$ mode $b$ in waveguide $B$ ) as a function of the propagation distance are given by [18]

$$
\begin{gathered}
\tilde{A}(z)=\tilde{A}(0)\left(\cos \left(\beta_{c} z\right)-\frac{i \delta}{\beta_{c}} \sin \left(\beta_{c} z\right)\right) e^{i \delta z} \\
\tilde{B}(z)=\tilde{A}(0)\left(\frac{i \kappa_{b a}}{\beta_{c}} \sin \left(\beta_{c} z\right)\right) e^{-i \delta z} \\
A(z)=\tilde{A}(z) e^{i \kappa_{a z} z} \\
B(z)=\tilde{B}(z) e^{i \kappa_{b b} z}
\end{gathered}
$$

where $\kappa_{a a}$ and $\kappa_{b b}$ are the self-coupling coefficients that take into account the variation in the propagation constant of each mode due to the perturbation caused by the other waveguide, $\kappa_{a b}$ and $\kappa_{b a}$ are the mutual coupling coefficients between both modes which depend on the waveguide separation, $g$. Finally, $\beta_{c}$ and $\delta$, the phase mismatch, can be expressed as

$$
\begin{gathered}
2 \delta=\left(\beta_{b}+\kappa_{b b}\right)-\left(\beta_{a}+\kappa_{a a}\right) \\
\beta_{c}=\sqrt{\kappa_{a b} \kappa_{b a}+\delta^{2}}
\end{gathered}
$$

where $\beta_{a}$ and $\beta_{b}$ are the propagation constants of modes $a$ and $b$ at their respective unperturbed waveguides. The complete field profile across the directional coupler can be obtained as a combination of the two mode fields as

$$
E(r)=\tilde{A}(0)\left(\varepsilon_{1}(x, y) e^{i \beta_{1} z}+\varepsilon_{2}(x, y) e^{i \beta_{2} z}\right)
$$

taking into account that

$$
\begin{aligned}
& \varepsilon_{1}(x, y)=\frac{\left(\beta_{c}-\delta\right) \hat{\varepsilon}_{a}(x, y)+\kappa_{b a} \hat{\varepsilon}_{b}(x, y)}{2 \beta_{c}} \\
& \varepsilon_{2}(x, y)=\frac{\left(\beta_{c}+\delta\right) \hat{\varepsilon}_{a}(x, y)-\kappa_{b a} \hat{\varepsilon}_{b}(x, y)}{2 \beta_{c}}
\end{aligned}
$$


where $\hat{\varepsilon}_{a}(x, y)$ and $\hat{\varepsilon}_{b}(x, y)$ are the normalized mode profiles of both modes in their respective isolated waveguides and

$$
\begin{gathered}
\beta_{1}=\bar{\beta}+\beta_{c} \\
\beta_{2}=\bar{\beta}-\beta_{c} \\
2 \bar{\beta}=\left(\beta_{b}+\kappa_{b b}\right)+\left(\beta_{a}+\kappa_{a a}\right)
\end{gathered}
$$

The total field in the coupler is a linear combination of two independent patterns $\varepsilon_{1}(x, y)$ and $\varepsilon_{2}(x, y)$ propagating with different propagation constants $\beta_{1}$ and $\beta_{2}$. These modes of the two-waveguide structure of the directional coupler are known as the supermodes of the structure and an example of their profile are shown in Figure 4.2.3 for both strip and ridge structures. In this figure, the magnitude of the field profile of the even and odd supermodes in the center of the guides $(y=0)$ along the $x$ direction are shown with a blue solid line. The main characteristics of the supermodes depend on $\delta, \kappa_{a b}$ and $\kappa_{b a}$.
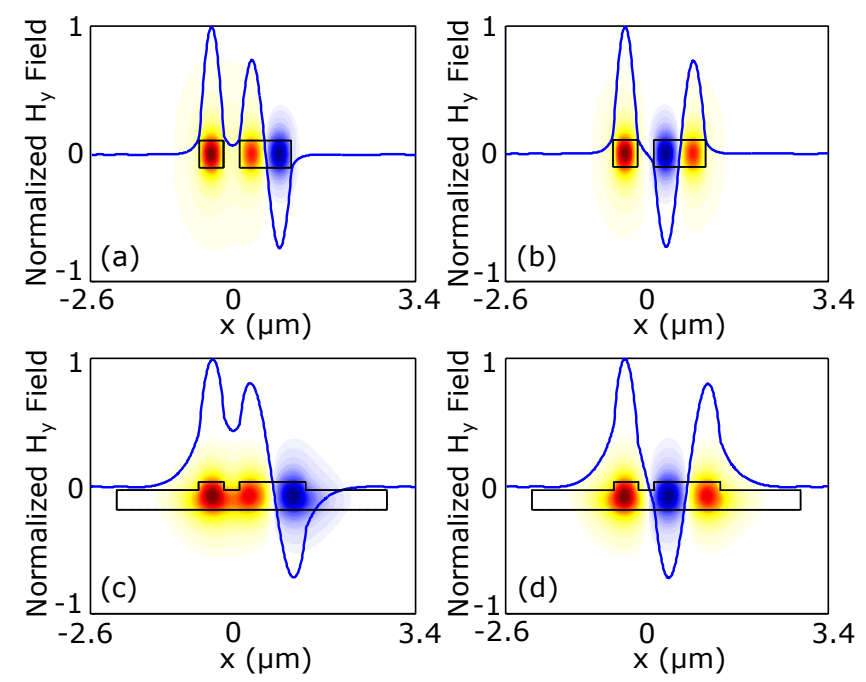

Figure 4.2.3. Normalized $H_{y}$ field of even and odd supermodes in the ADC for $T E_{0}$ and $T E_{1}$ modes. (a) Even and (b) odd supermodes in strip structure (c) Even and (d) odd supermodes in ridge structure.

From equation (4.2.2) the coupling efficiency from waveguide $A$ to waveguide $B$ in the ADC can be expressed as

$$
K=\frac{\left|\kappa_{b a}\right|^{2}}{\beta_{c}^{2}} \sin ^{2}\left(\frac{\pi \Delta n}{\lambda} L\right)
$$

where $\Delta n$ is the effective index difference between the even and odd supermodes; $\lambda$ is the wavelength; and $L$ is the length of the coupler.

If the modes to be coupled are phase-matched, then $\delta=0$ and $\kappa_{a b}=\kappa_{b a}^{*}$ the coupling efficiency is maximized,

$$
K=\sin ^{2}\left(\frac{\pi \Delta n}{\lambda} L\right)
$$

In this case, if we consider $\alpha$ as an arbitrary geometric parameter (i.e., $\alpha$ can be replaced by $w, g, h$, or $t$ ), for the minimum coupling length, $L$, in order to achieve $K=1$, the sensitivity of the coupling efficiency to this parameter can be expressed as [17]

$$
\frac{\partial K}{\partial \alpha}=2 \sin ^{-1}(\sqrt{K}) \sqrt{K(1-K)} \frac{1}{\Delta n} \frac{\partial \Delta n}{\partial \alpha}
$$


The sensitivity of the coupling efficiency is directly determined by the sensitivity parameter $(\partial \Delta n / \partial \alpha) / \Delta n$. A robust ADC design should consider the fabrication tolerances in the different device dimensions in order to optimize the fabrication process yield.

The common material used in the investigation of several integrated applications is the silicon on insulator wafer with a $220 \mathrm{~nm}$ silicon thickness [19]. The refractive indices of $\mathrm{Si}$ and $\mathrm{SiO}_{2}$ at $1550 \mathrm{~nm}$ are $n_{S i}=3.4764$ and $n_{S_{S O O}}=1.4440$, respectively, and the eigenmodes' effective indexes in the waveguides will be calculated with the three-dimensional (3D) beam propagation method (3D-BPM). For this height, the TE polarization is usually preferred for its lower bending losses [19].

Two structure types will be considered in the ADC coupler design, strip and ridge. In the strip structure a typical width for a single mode waveguide is $w_{A}=450 \mathrm{~nm}$ with an effective index value of $n_{\text {eff }}$ TEO $=2.4111$ at the wavelength design, $1550 \mathrm{~nm}$. The width of the two-mode waveguide must be selected to obtain a similar value for the effective index of the $\mathrm{TE}_{1}$ mode $\left(w_{B}=962 \mathrm{~nm}\right.$ for an unperturbed waveguide) so the phase-matched condition is fulfilled. The effective index value for the ridge $(t=150 \mathrm{~nm})$ single mode waveguide is $n_{\text {eff }}$ TEO $=2.6689$ when $w_{A}=450 \mathrm{~nm}$ and the corresponding width for the two-mode waveguide should be $w_{B}=1227 \mathrm{~nm}$ if an isolated waveguide is considered.

Supermodes permit to analyze the sensitivity of the ADC coupling efficiency by calculating the $\Delta n$ between the even and odd supermodes. The effective indexes of the even and odd supermodes have been also calculated with the 3D-BPM method.

Different designs have been considered ( $W_{A}$ from $430 \mathrm{~nm}$ to $470 \mathrm{~nm}$ and $g=100 \mathrm{~nm}, 200 \mathrm{~nm}$, $300 \mathrm{~nm}$ and $400 \mathrm{~nm}$ (only for the ridge design)) and for each case the two-mode width, $w_{B}$, has been selected in order to fulfil the phase-matched condition, $\delta=0$, when the influence of both waveguides are taken into account (equation (4.2.5)). Small variations of all parameters $(w, g, h$ and $t$ ) have been introduced in the simulations in order to analyze the robustness of the ADC against fabrication tolerances.

If the waveguide width is increased, the modes would be more confined in the center of the waveguide, which would reduce $\Delta n$. However, $\Delta n$ is also dependent on the gap between waveguides; therefore, a decrease in the gap, $g$, increases the mode overlap between the two waveguides and consequently $\Delta n$. If the variations in the waveguide widths are perfectly anti correlated with the gap variations, that is to say, the width variations are supposed to be symmetric with respect to their centerlines, a robust coupler design must fulfilled

$$
\frac{\partial \Delta n}{\partial w}-\frac{\partial \Delta n}{\partial g} \approx 0
$$

expression that was used also in [17] to select the most tolerant dimensions of symmetric DC and where a significant anti correlation between width and gaps variations was found in the device fabrication (correlation coefficient, $\rho=-0.6$ ). 

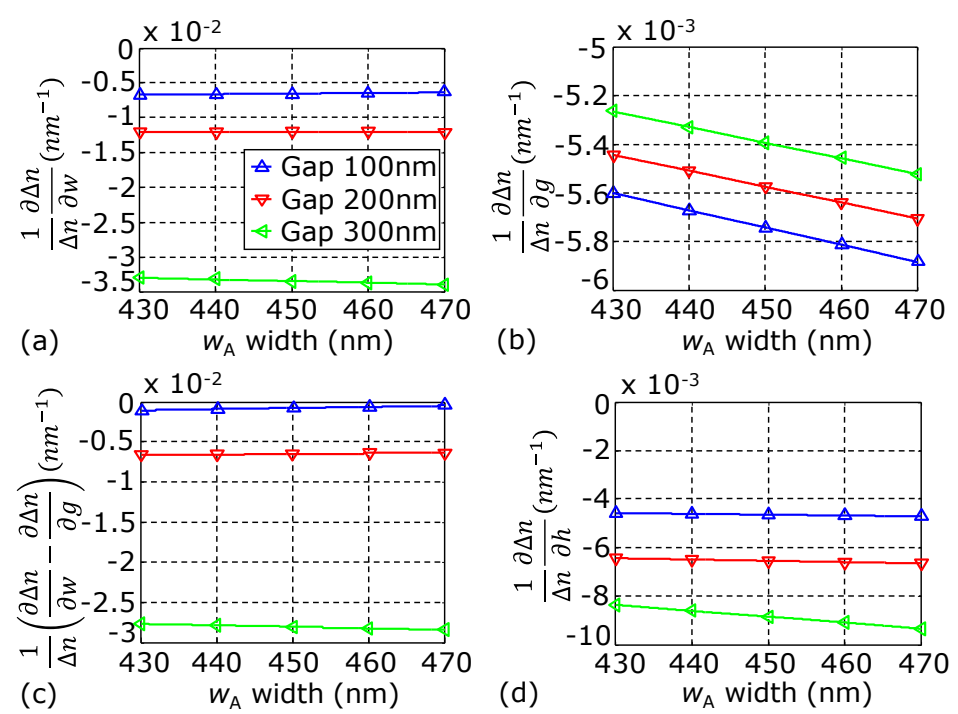

Figure 4.2.4. Fractional change in $\Delta n$ for strip structure with respect to (a) waveguide width, $w$,

(b) waveguide gap, $g$, (c) correlated changes in $w$ and $g$, and (d) waveguide height, $h$.

Figure 4.2.4 depicts the simulated sensitivity parameter for the width, gap and height variations in the strip structure at $1550 \mathrm{~nm}$. From Figure 4.2.4(a) it can be stated that in terms of sensitivity to width variations the $100 \mathrm{~nm}$ gap offers a more robust design than the configurations with a wider gap $(200 \mathrm{~nm}$ and $300 \mathrm{~nm})$. In fact, for the nominal design $\left(w_{A}=450 \mathrm{~nm}\right.$ and $\left.w_{B}=969 \mathrm{~nm}\right)$, the $100 \mathrm{~nm}$ gap obtains a value of $-0.64 \cdot 10^{-2} \mathrm{~nm}^{-1}$. However, the $200 \mathrm{~nm}$ and $300 \mathrm{~nm}$ gaps obtained worse values $\left(-1.2 \cdot 10^{-2} \mathrm{~nm}^{-1}\right.$ and $-3.3 \cdot 10^{-2} \mathrm{~nm}^{-1}$, respectively) for $w_{A}=450 \mathrm{~nm}$, being 2 and 5 times less robust than the $100 \mathrm{~nm}$ gap. If we consider the whole simulated $w_{A}$ width range $(430 \mathrm{~nm}$ to $470 \mathrm{~nm})$, the sensitivity to variations in the waveguides width remains practically constant for a fixed gap value. However, in terms of sensitivity to the gap dimension (Figure 4.2.4(b)), the sensitivity worsens slightly when the width increases. The difference between all gaps is quite small obtaining the best response for the $300 \mathrm{~nm}$ gap, as can be seen in Figure 4.2.4(b). We have not considered smaller gaps than $100 \mathrm{~nm}$ due to the additional difficulty in the fabrication process and the increase of the $\mathrm{TE}_{0}$ coupling to the TMF, which may result in a higher crosstalk level $(-23.4 \mathrm{~dB}$ at $1550 \mathrm{~nm}$ for $g=100 \mathrm{~nm}[8])$.

In Figure 4.2.4(c), the simultaneous anti correlated variations in the width and gap parameters for the purpose of fulfilling equation (4.2.16) remark that the $100 \mathrm{~nm}$ gap achieves the optimum result with a value of $-0.074 \cdot 10^{-2} \mathrm{~nm}^{-1}$ for $w_{A}=450 \mathrm{~nm}$. However, the $200 \mathrm{~nm}$ and $300 \mathrm{~nm}$ gaps obtain a value of $-0.66 \cdot 10^{-2} \mathrm{~nm}^{-1}$ and $-2.8 \cdot 10^{-2} \mathrm{~nm}^{-1}$ being 9 and 38 times less robust than the $100 \mathrm{~nm}$ gap. As it happened with the widths variations, the sensitivity to anti correlated variations in width and gap dimensions remain almost constant when a design with different $w_{A}$ is considered ( $\pm 20 \mathrm{~nm}$ respect to $\left.w_{A}=450 \mathrm{~nm}\right)$. Therefore, the selection of the $w_{A}$ parameter is not critical. In Figure 4.2.4(d) the sensitivity to height variations is depicted and the best response is again achieved for the $100 \mathrm{~nm}$ gap. The magnitude order of the sensitivity parameter is in the range of $10^{-3} \mathrm{~nm}^{-1}$ (as it happens with gap variations), whilst it is an order of magnitude higher in the case of the width and gap variations. Consequently, more attention must be paid to the width and gap dimension tolerances during the design process. 

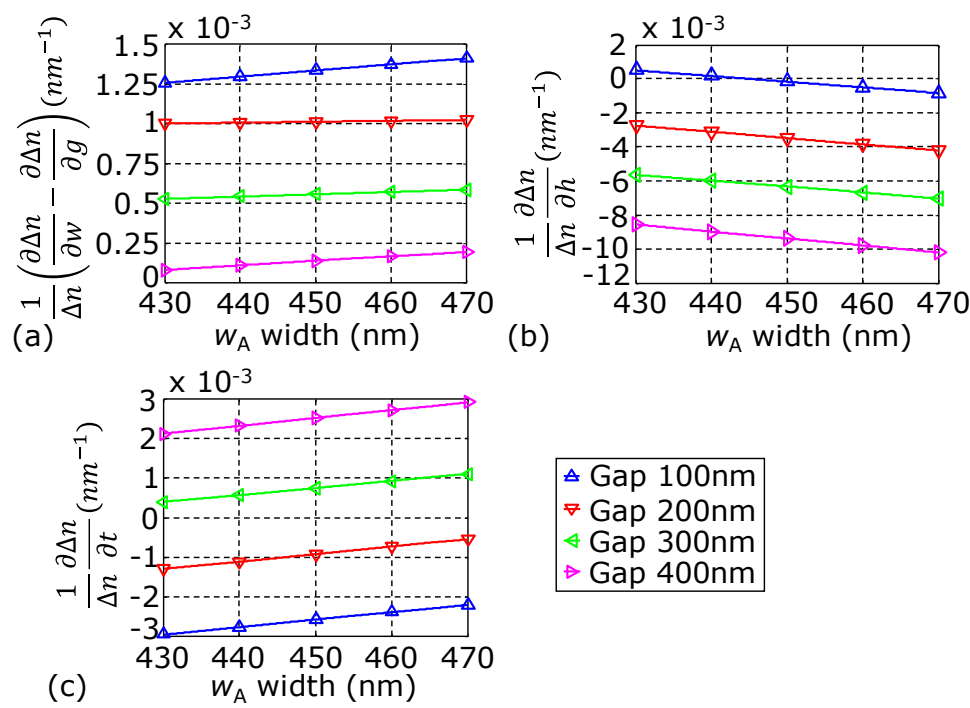

$\star$ Gap 100nm
$*$ Gap 200nm
$\star$ Gap 300nm
$\star$ Gap 400nm

Figure 4.2.5. Fractional change in $\Delta n$ for ridge structure with respect to (a) correlated changes in $w$ and $g$, (b) waveguide height, $h$ and (c) slab thickness, $t$.

The ADC with ridge structure was simulated considering the same parameters that were used in the strip simulations, but now including a new parameter, the slab thickness, $t$. In Figure 4.2.5(a), the sensitivity to the anti-correlated width and gap variations is shown. The best response was obtained for the highest gap $(400 \mathrm{~nm})$ with a value of $0.14 \cdot 10^{-3} \mathrm{~nm}^{-1}$ $\left(w_{A}=450 \mathrm{~nm}\right.$ and $\left.w_{B}=1223 \mathrm{~nm}\right)$. For the rest of gaps, the different values were $0.55 \cdot 10^{-3} \mathrm{~nm}^{-1}$, $1 \cdot 10^{-3} \mathrm{~nm}^{-1}$ and $1.3 \cdot 10^{-3} \mathrm{~nm}^{-1}$ being 4,7 and 10 times less robust than the $400 \mathrm{~nm}$ gap, respectively. If we consider a range of $\pm 20 \mathrm{~nm}$ around the nominal design widths, the sensitivity parameter of all gaps remain practically constant with small variations. The difference between all gaps has significantly decreased in comparison with the strip structure. However, as shown in Figure 4.2.5(b), the sensitivity to height variation presents the opposite behavior and the smallest gap $(100 \mathrm{~nm})$ has the best sensitivity parameter with a value of $-0.1 \cdot 10^{-3} \mathrm{~nm}^{-1}$ in the nominal design. In both SOI structures (strip and ridge) the optimum result is achieved for the minimum gap.

In Figure 4.2.5(c), the simulated sensitivity parameter for the slab thickness variation is shown. The optimum gap is achieved between two different gaps (200 nm and $300 \mathrm{~nm}$ ) depending on the $w_{A}$ width. When the nominal design is considered, the optimum gap is $300 \mathrm{~nm}$ with a value of $0.7 \cdot 10^{-3} \mathrm{~nm}^{-1}$ unlike the $200 \mathrm{~nm}$ gap that obtained a value of $-0.9 \cdot 10^{-}$ $3 \mathrm{~nm}^{-1}$. For $w_{A}$ widths lower than $450 \mathrm{~nm}$ is better to choose the $300 \mathrm{~nm}$ gap whilst for broader widths, it is better to select the $200 \mathrm{~nm}$ gap.

From the results obtained in Figure 4.2.5, it can be stated that the optimum gap is the $400 \mathrm{~nm}$ considering the anti-correlated changes in width and gap, the $100 \mathrm{~nm}$ gap for changes in height and $300 \mathrm{~nm}$ (when $w_{A}$ is $450 \mathrm{~nm}$ ) for changes in the slab thickness. However, in SOI fabrication process, the most critical parameter is the width and gap variation [20], therefore, the optimum ridge design is achieved when the $400 \mathrm{~nm}$ gap is selected. According to the trend shown in Figure 4.2.5(a), a $500 \mathrm{~nm}$ gap would obtain similar values of the sensitivity parameter module against $w$ and $g$ variations but offering a lesser compact design. Furthermore, the sensitivity will be worst against $h$ and $t$ variations following the trend shown in Figure 4.2.5(b) and Figure 4.2.5(c).

The proposed designs (100 nm gap for the strip structure and $400 \mathrm{~nm}$ gap for the ridge structure) for the ADCs are optimum in order to convert and (de)multiplex the $\mathrm{TE}_{0}$ mode into the $\mathrm{TE}_{1}$ mode taking into account the width and gap variations produced in the nominal design due to the fabrication tolerances. However, the ridge structure is more tolerant than the strip structure by a factor of $6\left(0.14 \cdot 10^{-3} \mathrm{~nm}^{-1}\right.$ by $-0.074 \cdot 10^{-2} \mathrm{~nm}^{-1}$, respectively), a similar performance as the obtained for the symmetric directional couplers [17]. 


\section{REFERENCES}

[1] "Cisco Visual Networking Index: Forecast and Methodology, 2016-2021," Cisco White Paper, https://goo.gl/DXQUFE

[2] R.-J. Essiambre, G. Kramer, P. J. Winzer, G. J. Foschini, and B. Goebel, J. Lightwave Technol. 28, 662 (2010).

[3] D. J. Richardson, Philos. Trans. R. Soc. A 374, 20140441 (2016).

[4] T. Mizuno, H. Takara, A. Sano, and Y. Miyamoto, J. Lightwave Technol. 34, 582 (2016).

[5] P. J. Winzer, Nat. Photonics 8, 345 (2014).

[6] D. Melati, A. Alippi, A. Annoni, N. Peserico, and A. Melloni, Opt. Lett. 42, 342 (2017).

[7] D. Garcia-Rodriguez, J. L. Corral, and R. Llorente, IEEE Photon. Technol. Lett. 29, 929 (2017).

[8] D. Garcia-Rodriguez, J. L. Corral, A. Griol, and R. Llorente, Opt. Lett. 42, 1221 (2017).

[9] Y.-S. Lee, Z. Wang, Z.-Y. Li, Y. Li, Q. Li, C. Cui, and C.-Q. Wu, Opt. Commun. 406, 173 (2018).

[10] C. Sun, Y. Yu, M. Ye, G. Chen, and X. Zhang, Sci. Rep. 6, 38494 (2016).

[11] N. Hanzawa, K. Saitoh, T. Sakamoto, T. Matsui, K. Tsujikawa, T. Uematsu, and F. Yamamoto, J. Lightwave Technol. 33, 1161 (2015).

[12] Y. Yamashita, T. Fujisawa, S. Makino, N. Hanzasa, T. Sakamoto, T. Matsui, K. Tsujikawa, F. Yamamoto, K. Nakajima, and K. Saitoh, J. Lightwave Technol. 35, 2252 (2017).

[13] A. Zanzi, A. Brimont, A. Griol, P. Sanchis, and J. Marti, Opt. Lett. 41, 227 (2016).

[14] N. Hanzawa, K. Saitoh, T. Sakamoto, T. Matsui, K. Tsujikawa, M. Koshiba, and F. Yamamoto, Opt. Express 22, 29321 (2014).

[15] J. Wang, S. He, and D. Dai, Laser Photon. Rev. 8, L18 (2014).

[16] C. Pan and B. M. A. Rahman, J. Lightwave Technol. 34, 2288 (2016).

[17] J. C. Mikkelsen, W. D. Sacher, and J. K. S. Poon, Opt. Express 22, 3145 (2014).

[18] J. M. Liu, Photonic Devices (Cambridge University, 2007).

[19] D. X. Xu, J. H. Schmid, G. T. Reed, G. Z. Mashanovich, D. J. Thomson, M. Nedeljkovic, X. Chen, D. Van Thourhout, S. Keyvaninia, and S. K. Selvaraja, IEEE J. Sel. Top. Quantum Electron. 20, 422 (2014).

[20] J. L. Corral, D. Garcia-Rodriguez, and R. Llorente, IEEE Photon. Technol. Lett. 28, 425 (2016). 


\title{
4.3 Bimodal grating coupler design on SOI technology for mode division multiplexing at $1550 \mathrm{~nm}$
}

\section{BIBLIOGRAPHY RECORD}

- Authors: David Garcia-Rodriguez, Juan L. Corral, Amadeu Griol and Roberto Llorente

- Publication: Optics Express, vol. 26, no. 15

- Year: 2018

- Pages: 19445-19455

- OCIS codes: (050.2770) Gratings; (060.1810) Buffers, couplers, routers, switches, and multiplexers; (060.4230) Multiplexing; (130.3120) Integrated optics devices; (230.7400) Waveguides, slab.

\begin{abstract}
In this paper, we evaluate by means of simulation and experimentally the simultaneous coupling of the $L P_{01 x}-L P_{11 a x}$ fiber modes and the $T E_{0}-T E_{1}$ nanophotonic SOI waveguide modes using a grating coupler for a two mode fiber at $1550 \mathrm{~nm}$. Both the grating width (ranging from $10 \mu \mathrm{m}$ to $15 \mu \mathrm{m}$ ) and the grating vertical profile have been considered in the design procedure. The optimum design ( $14 \mu \mathrm{m}$ width and $609 \mathrm{~nm}$ grating period) has been selected in terms of coupling efficiency (both $\mathrm{LP}_{01 \mathrm{x}}-\mathrm{TE}_{0}$ and $\mathrm{LP}_{11 \mathrm{ax}}-\mathrm{TE}_{1}$ ), compactness and tolerance to lateral misalignments between fiber and coupler. The $\mathrm{LP}_{01 \mathrm{x}}-\mathrm{TE}_{0}$ and $\mathrm{LP}_{11 \mathrm{ax}}-\mathrm{TE}_{1}$ modes achieved coupling efficiencies of $49 \%$ and $45 \%$, respectively.
\end{abstract}




\section{INTRODUCTION}

In the last decade, the data traffic demand has been continuously increasing due to new internet based services, particularly video streaming and machine to machine communications, driving optical communication systems based on single-mode fibers to their capacity limit [1]. Spatial division multiplexing (SDM) or mode division multiplexing (MDM) are promising techniques to overcome the capacity limit of the optical fiber [1]. In its simplest implementation, MDM permits to propagate the $\mathrm{LP}_{01}$ and $\mathrm{LP}_{11}$ modes in a two mode fiber (TMF) [2].

Silicon-on-insulator (SOI) or planar lightwave circuit (PLC) are key technologies to provide low-cost photonic integrated mode converters and mode (de)multiplexers. Different techniques have been proposed to convert and (de)multiplex the modes; for example, asymmetrical directional couplers (ADC), multimode interference (MMI), tapered directional coupler, adiabatic couplers, inverse tapers or trident couplers [3-14].

An important aspect in the different integrated devices for MDM is the coupling to the optical fiber. Depending on the employed technology, a vertical or horizontal coupling is required. On one hand, PLC favors the use of the horizontal coupling due to the similar dimensions between the waveguide and the fiber core. This technology offers low loss and mass productivity due to their mature manufacturing technology. However, the main drawback are the higher sizes and higher bending radius (above $5 \mathrm{~cm}[15]$ ). On the other hand, SOI technology offers lower size, better repeatability and higher robustness than the PLC based devices but the coupling to the fiber is more demanding.

When a few mode fiber (FMF) is under consideration the coupling of the different modes to/from the SOI waveguides must be solved. SOI with horizontal coupling are traditional based on spot size converter which were limited to the fiber fundamental mode [13]. Recently some modifications have been proposed in order to combine several inverse taper spot size converters by means of $\mathrm{Y}$-junction or trident waveguides to couple to fiber higher modes [13, 14]. The inverse tapers provide highly efficient, polarization insensitive, and broad bandwidth optical coupling but they require a high alignment precision, a complex design and long lengths $[13,16]$. Besides, a single horizontal coupler is required for each fiber mode to couple.

On the other hand, vertical coupling in SOI technology is based on the use of grating couplers. Grating couplers have been successfully used to couple the $\mathrm{TE}_{0}$ mode of the SOI waveguide to the $L \mathrm{P}_{01}$ mode of the standard single-mode fiber (SSMF), and vice versa [17-19]. Although grating couplers are easy to fabricate and provide on chip test measurements with small footprints, the main drawbacks are the limited bandwidth and their polarization sensitivity.

One first approach to vertically couple light from the SOI waveguide to the $L P_{01} / L P_{11}$ modes in a FMF was based on the use of one 2D grating coupler excited from two 90 degrees apart waveguides propagating the $T E_{0}$ and $T E_{1}$ modes [20]. Other proposed SOI devices are based on the use of $2 \mathrm{D}$ gratings simultaneously excited from several waveguides propagating the $\mathrm{TE}_{0}$ modes but with a fixed phase relation between the waveguide modes [21-23]. These devices offer the generation of up to six fiber modes (all $L P_{01}$ and $\mathrm{LP}_{11}$ degenerated modes) but a more complex feeder circuit is required with a higher footprint and thermal tuning to adjust the phase shift at the different waveguides [21-23]. A strictly vertical coupling to the fiber is also required on these devices with the corresponding issues of second order reflection at the waveguide fiber interface [20-23].

Thus, using a single grating coupler that couples simultaneously the $\mathrm{TE}_{0}$ and $\mathrm{TE}_{1}$ modes from a single SOI waveguide to the $\mathrm{LP}_{01}$ and $\mathrm{LP}_{11}$ modes in the FMF would be a key component for low cost and low size integrated devices in the MDM systems.

In this letter, a grating coupler for the simultaneous coupling of the $\mathrm{TE}_{0}$ and $\mathrm{TE}_{1}$ modes into one single polarization of the $\mathrm{LP}_{01}$ mode and one single polarization and azimuthal orientation of the $\mathrm{LP}_{11}$ mode of the TMF is designed. Both the nominal performance of the grating coupler at the design wavelength and the sensibility of the coupling efficiency to lateral misalignment 
of the fiber are considered. The best grating design is selected in terms of both $T E_{0}-\mathrm{LP}_{01}$ and $\mathrm{TE}_{1}-\mathrm{LP}_{11}$ coupling. The selected device has been fabricated and measured.

\section{GRATING COUPLER DESIGN}

The general concept of the MDM link is depicted in Figure 4.3.1. It consists of two lasers emitting at $1550 \mathrm{~nm}$ propagating the $\mathrm{LP}_{01}$ mode in a standard single-mode fiber (SSMF). Both $\mathrm{LP}_{01}$ modes pass through a polarization controller to impinge the standard $L \mathrm{P}_{01}-\mathrm{TE}_{0}$ grating coupler with the s-polarization. The $\mathrm{TE}_{0}$ mode from the upper branch is converted into the $\mathrm{TE}_{1}$ mode and it is multiplexed with the optical signal coming from the lower branch, propagating both modes, $T E_{0}$ and $T E_{1}$, to the common output. The $\mathrm{TE}_{1}$ mode is excited and coupled to the TMF propagating the $\mathrm{LP}_{11 \mathrm{a}}$ mode with s-polarization. Finally, both modes are coupled to the two mode fiber (TMF) using a specific grating coupler, corresponding now to the fiber $\mathrm{LP}_{01}$ and $\mathrm{LP}_{11 \mathrm{a}}$ modes. At the receiver, the same device is used in order to demultiplex each mode to the corresponding photodiode. As in any other mode demultiplexer based on vertical grating couplers, the polarization of the fiber modes must be properly aligned to the s-polarization at the fiber waveguide interface. Depending on the mode coupling induced by the TMF link a multiple input multiple output (MIMO) digital processing stage could be required at the receiver to properly split both data streams.

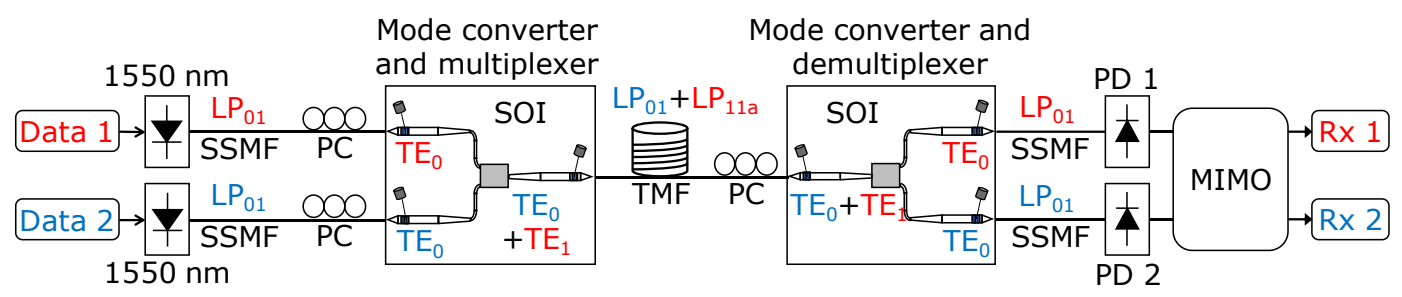

Figure 4.3.1. Scheme for mode division multiplexing (MDM) at $1550 \mathrm{~nm}$ with two mode fiber (TMF) optical transmission media.

\section{GRATING THEORY}

The periodic structures are defined by the Bragg condition that describes the relation between the wave vectors of the incident and diffracted waves. In the case of waveguide grating couplers, the incident wave is replaced by the guided mode of the waveguide, which is characterized by its propagation constant $\beta$. The equation can be defined as [24]

$$
\frac{2 \pi}{\lambda} n_{\text {cover }} \sin \left(\theta_{\mathrm{m}}\right)=\frac{2 \pi}{\lambda} n_{\text {eff }}+m \frac{2 \pi}{\Lambda}
$$

where $\lambda$ is the design wavelength, $n_{\text {cover }}$ is the refractive index of the cover substrate, $\theta_{m}$ is the tilt angle, $n_{\text {eff }}$ the effective index of the optical mode in the grating waveguide, $\Lambda$ is the grating period and the integer $m$ is the diffraction order.

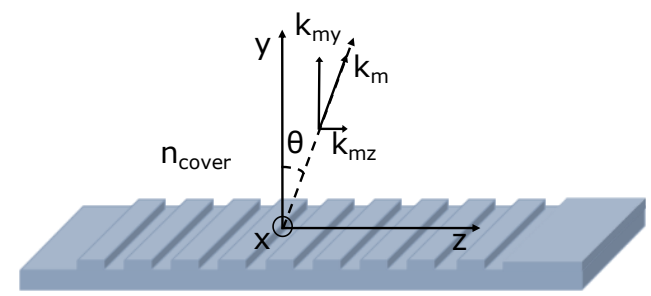

Figure 4.3.2. Schematic waveguide grating coupler diagram for coupling light to/from an optical fiber.

Considering the grating as an output coupler, the coupling to the optical fiber is done for the first diffraction order $(m=-1)$ with a specific tilted angle $(\theta)$, as depicted in Figure 4.3.2. In the case of simultaneous $\mathrm{TE}_{0}$ and $\mathrm{TE}_{1}$ modes coupling, equation (4.3.1) must be fulfilled in both cases, therefore, the effective indexes for both modes need to be approximately equal. If the waveguide widths are sufficiently large, the $T E_{0}$ and $T E_{1}$ modes are expected to have similar effective indexes. 
The coupling efficiency from fiber to waveguide is the same as from waveguide to fiber due to reciprocity theorem. When the electromagnetic fields at the position of the fiber facet are known, the coupling efficiency to fiber becomes [25]

$$
\eta=\left|\iint_{S} E \times H_{\text {fib }}^{*} d S\right|^{2}
$$

where $E$ is the normalized electrical field of the diffracted wave from the grating, and $H_{\text {fib }}$ is the normalized magnetic fields of the optical mode in the fiber and $S$ is the fiber facet. Equation (4.3.2) is a good approximation, even if the grating is covered by air instead of silica [26].

When the optical mode of the waveguide is launched from the grating, some light is refracted upwards and the rest is refracted downwards (across the substrate), due to the vertical grating symmetry. Thus, for this design the efficiency will not be higher than $50 \%$ unless reflective elements are used $[17,24]$.

The complete coupler problem is a 3D problem but can be approximated by two 2D problem due to the highly different dimensions between the waveguide width and height where the width is much larger than the height. In Figure 4.3.3, the fundamental mode $\left(\mathrm{TE}_{0}\right)$ and the first higher order mode ( $\left.\mathrm{TE}_{1}\right)$ for a $12 \mu \mathrm{m}$ SOI waveguide width are shown.
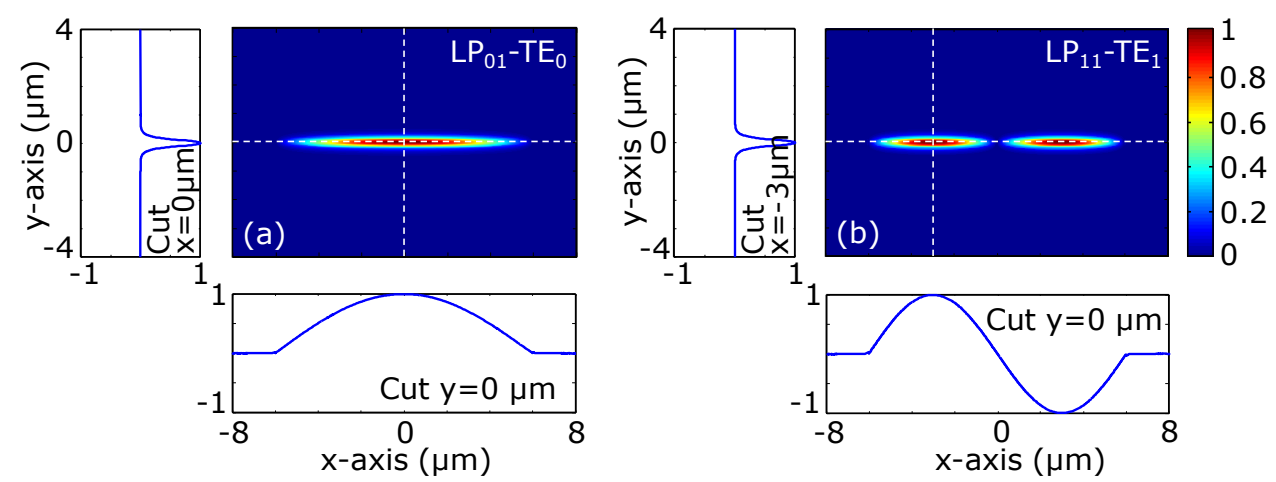

Figure 4.3.3. $2 D$ Near-field pattern and $1 D x-y$ cuts for the $T E_{0}$ and $T E_{1}$ modes considering a $12 \mu m$ wide and $220 \mathrm{~nm}$ high SOI waveguide. (a) TE mode with $x=0 \mu \mathrm{m}$ and $y=0 \mu \mathrm{m}$ cuts, (b) TE mode with $x=-3 \mu \mathrm{m}$ and $y=0 \mu \mathrm{m}$ cuts.

The complete coupler problem is a 3D problem but can be approximated by two 2D problem due to the highly different dimensions between the waveguide width and height where the width is much larger than the height. In Figure 4.3.3, the fundamental mode $\left(\mathrm{TE}_{0}\right)$ and the first higher order mode $\left(\mathrm{TE}_{1}\right)$ for a $12 \mu \mathrm{m}$ SOI waveguide width are shown.

\section{GRATING DESIGN}

As previously commented, the complete coupler design problem can be divided into two 2D problems. Firstly we will assess the width of the grating coupler by taking into account the overlap between the mode field distributions in the transverse planes of both the SOI waveguide and the FMF. The most common grating coupler design based on SOI technology $\left(\mathrm{SiO}_{2}\right.$ layer height $=220 \mathrm{~nm}$ ) for the $\mathrm{TE}_{0}-\mathrm{LP}_{01}$ coupling to SMF-28 fiber has a waveguide width $\left(w_{g}\right)$ of $12 \mu \mathrm{m}$ [17]. If we take a TMF at $1550 \mathrm{~nm}$ such as SM2000 fiber from Thorlabs $\left(11 \mu \mathrm{m}\right.$ core diameter with a $\mathrm{SiO}_{2}$ cladding, $\mathrm{n}_{\text {core }}=1.4495$ at $\left.1550 \mathrm{~nm}\right)$, the $\mathrm{LP}_{01}$ mode profile is slightly wider than the LP ${ }_{01}$ mode profile for the SMF-28 $(11 \mu \mathrm{m}$ in SM2000 vs. $10.4 \mu \mathrm{m}$ in SMF-28). Besides, the $T E_{1}$ mode needs to be coupled to the $L P_{11}$ mode and, as shown in Figure 4.3.4, the $\mathrm{LP}_{11}$ mode profile in the $\mathrm{SM} 2000$ fiber is much wider than the $\mathrm{TE}_{1}$ mode profile in the $12 \mu \mathrm{m}$ waveguide. If the peak of the modes are taken as a reference, the $\mathrm{LP}_{11}$ mode is $1.64 \mu \mathrm{m}$ wider than the $\mathrm{TE}_{1}$ mode. Therefore, the optimum waveguide width is expected to be $1 \mu \mathrm{m}$ to $2 \mu \mathrm{m}$ greater than $12 \mu \mathrm{m}$. 

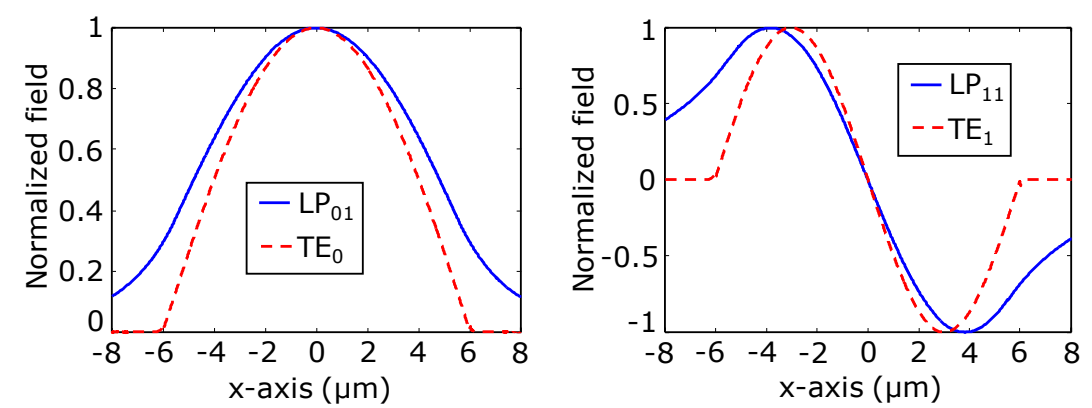

Figure 4.3.4. Comparison between the normalized horizontal field profile of the $L P_{01}$ and $L P_{11}$ modes from SM2000 fiber and the $T E_{0}$ and $T E_{1}$ modes for a SOI waveguide (height $=220 \mathrm{~nm}$ and waveguide width $=12 \mu \mathrm{m}$ ).

The grating coupler is composed by a $\mathrm{Si}$ core $\left(t_{\text {core }}\right)$, a $\mathrm{SiO}_{2}$ cladding ( $\left.t_{\text {cladding }}\right)$ and a $\mathrm{Si}$ substrate $\left(t_{\text {substrate }}\right)$, as can be seen in Figure 4.3.5. We use SOI wafers of $220 \mathrm{~nm} / 2 \mu \mathrm{m} \mathrm{Si} / \mathrm{SiO}_{2}$ layers

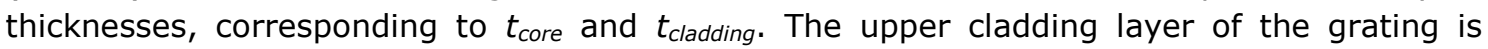
supposed to be air. The main parameters in the grating coupler design are the filling factor or duty cycle $(f f=w / \Lambda)$, the grating period $(\Lambda)$ and the etching depth $\left(e_{d}\right)$. Some typical values for the $\mathrm{TE}_{0}-\mathrm{LP}_{01}$ coupling using SMF 28 are: $f f=0.5, e_{d}=70 \mathrm{~nm}$ and $\Lambda=630 \mathrm{~nm}$, to maximize coupling efficiency at $1550 \mathrm{~nm}$ [26]. The incident angle $(\theta)$ is around 10 degrees in order to optimize the coupling efficiency and avoid reflections.

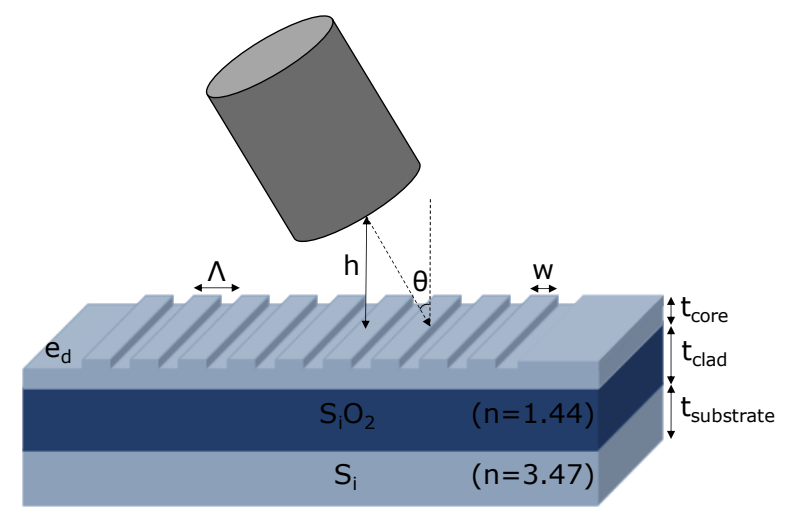

Figure 4.3.5. Grating coupler scheme based on SOI technology with an input fiber.

Figure 4.3.6 shows the simulation results of the coupling efficiency for the $\mathrm{LP}_{01}-\mathrm{TE}_{0}$ and $\mathrm{LP}_{11}-\mathrm{TE}_{1}$ modes with respect to the grating period and the filling factor. The results shown in this figure have been obtained according to the simulation approach described in section 3 for a $12 \mu \mathrm{m}$ waveguide width. Figure 4.3.6(a) results show a maximum efficiency region corresponding to an almost vertical line with optimum grating periods ranging from $607 \mathrm{~nm}$ to $611 \mathrm{~nm}$ when the filling factor varies from 0.48 to 0.52 , respectively. From all these possible design parameters the optimum grating period, $\Lambda=609 \mathrm{~nm}$, for a filling factor of $f f=0.5$ was selected. A similar performance is shown in Figure 4.3.6(b) for the $\mathrm{TE}_{1}-\mathrm{LP}_{11}$ coupling. Figure 4.3.6 was also simulated for different grating widths ( $w_{g}=10 \mu \mathrm{m}$ to $\left.15 \mu \mathrm{m}\right)$ with no influence on the results shown for $w_{g}=12 \mu \mathrm{m}$. Once the filling factor and the grating period were fixed the sensitivity to variations of the etching depth was found to be of less magnitude than the tolerance of the fabrication process in the vertical dimension so the typical $70 \mathrm{~nm}$ etching depth was not modified. 

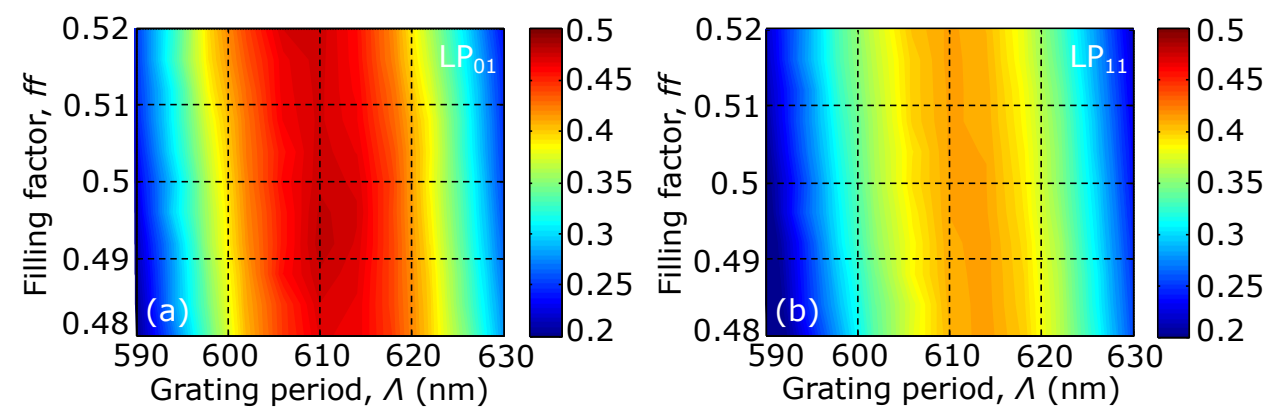

Figure 4.3.6. Coupling efficiency as a function of the grating period and filling factor for: (a) $L P_{01}-T E_{0}$ modes and $(b) L P_{11}-T E_{1}$ modes coupling.

Once the vertical dimensions of the grating coupler are fixed $\left(t_{\text {core }}=220 \mathrm{~nm}, \mathrm{e}_{\mathrm{d}}=70 \mathrm{~nm}, \mathrm{ff}=0.5\right.$ ) the SOI mode indexes in the grating can be estimated as the average value between the effective indexes of the unperturbed $\left(t_{c o r e}=220 \mathrm{~nm}\right)$ and perturbed $\left(t_{c o r e}-e_{d}=150 \mathrm{~nm}\right)$ waveguides. For a $w_{g}=12 \mu \mathrm{m}$ grating width quite similar effective indexes ( $n_{\text {effTE }}=2.6920 \mathrm{vs}$ $n_{\text {effTE1 }}=2.6897$ ) are obtained. As aforementioned in the grating theory subsection, both modes effective indexes are quite similar so equation (4.3.1) is fulfilled simultaneously and the small index difference would translate to a negligible 0.13 degrees difference in the incident angle. Thus, the grating coupler can be used for simultaneous coupling.

\section{SimULATION}

Simulations of fiber-grating coupling has been performed using 3D Finite Difference Time Domain method (3D-FDTD). Eigenmodes' effective indices in waveguides and optical fibers are obtained by means three-dimensional (3D) finite element method (3D-FEM) and three-dimensional beam (3D) propagation method (3D-BPM), respectively.

FDTD method is appropriate to simulate reduced regions with fast speed and low memory requirements. Therefore, we optimize the fiber height above the grating $(h=1 \mu \mathrm{m})$ to reduce our simulation region. Complete grating coupler has been simulated at $1550 \mathrm{~nm}$ for different waveguide widths $(10 \mu \mathrm{m}, 11 \mu \mathrm{m}, 12 \mu \mathrm{m}, 13 \mu \mathrm{m}, 14 \mu \mathrm{m}$ and $15 \mu \mathrm{m})$. The refractive indexes of $\mathrm{Si}$ and $\mathrm{SiO}_{2}$ are taken as $n_{S i}=3.4758$ and $n_{\mathrm{SiO}_{2}}=1.4442$, respectively. $\mathrm{LP}_{01}$ and $\mathrm{LP}_{11}$ modes are excited from the optical fiber and coupled to the waveguide. In order to assess the sensitivity to eventual fiber to coupler misalignments, the center of the fiber can be displaced along the width of the grating from $-4 \mu \mathrm{m}$ to $4 \mu \mathrm{m}$ with respect to the center of the grating coupler. Results of the coupling efficiency are depicted in Figure 4.3.7.

Figure 4.3.7(a) displays the coupling efficiency (CE) from $L P_{01}$ to $T^{2}$ and the undesired coupling efficiency from $L P_{01}$ to $\mathrm{TE}_{1}$, or leakage, as a function of displacement. All coupling efficiencies are symmetric and slow varying with respect to the center of the grating coupler, remaining practically constant for minor variations (up to $\pm 1 \mu \mathrm{m}$ ). However, depending on the selected width, the coupling efficiency from $L P_{01}$ to $T E_{0}$ increases from $26.4 \%$ (for a shift of $4 \mu \mathrm{m}$ ) to as high as $45.9 \%$ (no misplacement) for the $10 \mu \mathrm{m}$ width or, from $29.3 \%$ to as high as $49.7 \%$, respectively, for the $15 \mu \mathrm{m}$ width. For the maximum coupling, the difference between both grating widths is $3.8 \%$. If a $1 \mathrm{~dB}$ penalty in the coupling efficiency due to lateral misplacement is considered $\mathrm{a} \pm 2.4 \mu \mathrm{m}$ ( $10 \mu \mathrm{m}$ coupler) to $\pm 2.6 \mu \mathrm{m}$ (15 $\mu \mathrm{m}$ coupler) alignment range are obtained. Besides that, the $\mathrm{LP}_{01}$ to $\mathrm{TE}_{1}$ leakage is null for all widths when the fiber and the grating are perfectly aligned, but it ranges from $8.4 \%\left(w_{g}=15 \mu \mathrm{m}\right)$ to $13.3 \%$ $\left(w_{g}=10 \mu \mathrm{m}\right)$ for the maximum misplacement considered. The best performance in terms of $\mathrm{LP}_{01}$ to $\mathrm{TE}_{0}$ coupling efficiency is obtained for $w_{g}=15 \mu \mathrm{m}$ with a $49.7 \%$ efficiency, value that would decrease for wider widths $\left(49.6 \%\right.$ and $49.1 \%$ for $w_{g}=16 \mu \mathrm{m}$ and $\left.w_{g}=17 \mu \mathrm{m}\right)$. With this result in mind, widths wider than $15 \mu \mathrm{m}$ were not taken into consideration in the simulation.

The simulation results for the coupling efficiency from $L P_{11}$ to $T E_{1}$ and the undesired $L P_{11}$ to $\mathrm{TE}_{0}$ leakage are shown in Figure 4.3.7(b). In this case, the different responses are also symmetric with respect to the center of the grating coupler, but the sensitivity to lateral 
misalignment is quite higher than in the $L P_{01}-T_{E}$ case. From $L P_{11}$ to $T E_{1}$, the coupling efficiency increases approximately from $0.2 \%$ (for a lateral displacement of $4 \mu \mathrm{m}$ ) to $34.8 \%$ (no misplacement) for the $10 \mu \mathrm{m}$ width or, from $3.5 \%$ to as high as $46.35 \%$, respectively, for the $15 \mu \mathrm{m}$ width. In this case, the maximum difference between both cases is $11.5 \%, 3$ times higher than for the $L P_{01}-T_{0}$ coupling. The alignment range for the same $1 \mathrm{~dB}$ coupling efficiency penalty is reduced to $\pm 1.25 \mu \mathrm{m}$ (10 $\mu \mathrm{m}$ coupler) to $\pm 1.35 \mu \mathrm{m}$ ( $15 \mu \mathrm{m}$ coupler) in this case. In terms of $\mathrm{LP}_{11}$ to $\mathrm{TE}_{0}$ leakage, the efficiency at the maximum misplacement ranges from $42.3 \%$ to $21.8 \%$ for the $10 \mu \mathrm{m}$ and $15 \mu \mathrm{m}$ widths, respectively. It can be stated that $\mathrm{LP}_{11}$ shows a remarkable dependence with the waveguide width and the lateral displacement.

Even though the coupling efficiency simulation results imply that the $w_{g}=15 \mu \mathrm{m}, \Lambda=609 \mathrm{~nm}$, $f f=0.5, e_{d}=70 \mathrm{~nm}$ grating coupler would be the best option in terms of coupling efficiency a $w_{g}=14 \mu \mathrm{m}$ width has been selected to be fabricated as the best compromise when the integrated device size is also considered. The improvement in coupling efficiency with the wider coupler $\left(0.1 \%\right.$ and $1.2 \%$ difference for $L P_{01}-T_{E}$ and $L_{11}-T_{E}$ coupling, respectively) is incremental and the increase of $1 \mu \mathrm{m}$ in width (9.3\% increase) would translate to a quite longer adiabatic taper, increasing the device footprint by the same $9.3 \%$ factor. It is important to point out that the final design is $2 \mu \mathrm{m}$ wider than the optimum design for the $L \mathrm{P}_{01}-\mathrm{TE}_{0}$ and SMF-28 fiber, according to the SOI state of art. In this conventional $\mathrm{LP}_{01}-\mathrm{TE}_{0}$ grating coupler design for SMF-28 fiber the selected width $\left(w_{g}=12 \mu \mathrm{m}\right)$ is also slightly narrower than the optimal theoretical width.
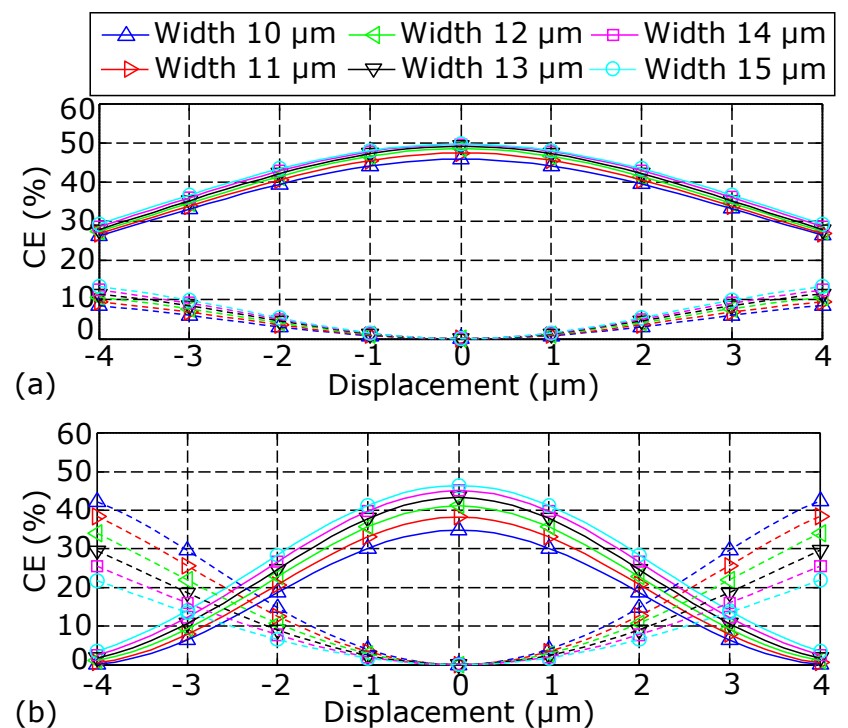

Figure 4.3.7. (a) Coupling efficiency from $L P_{01}$ to $T E_{0}$ (solid line) and from $L P_{01}$ to $T E_{1}$ (dashed line) and (b) Coupling efficiency from $L P_{11}$ to $T E_{1}$ (solid line) and from $L P_{11}$ to $T E_{0}$ (dashed line).
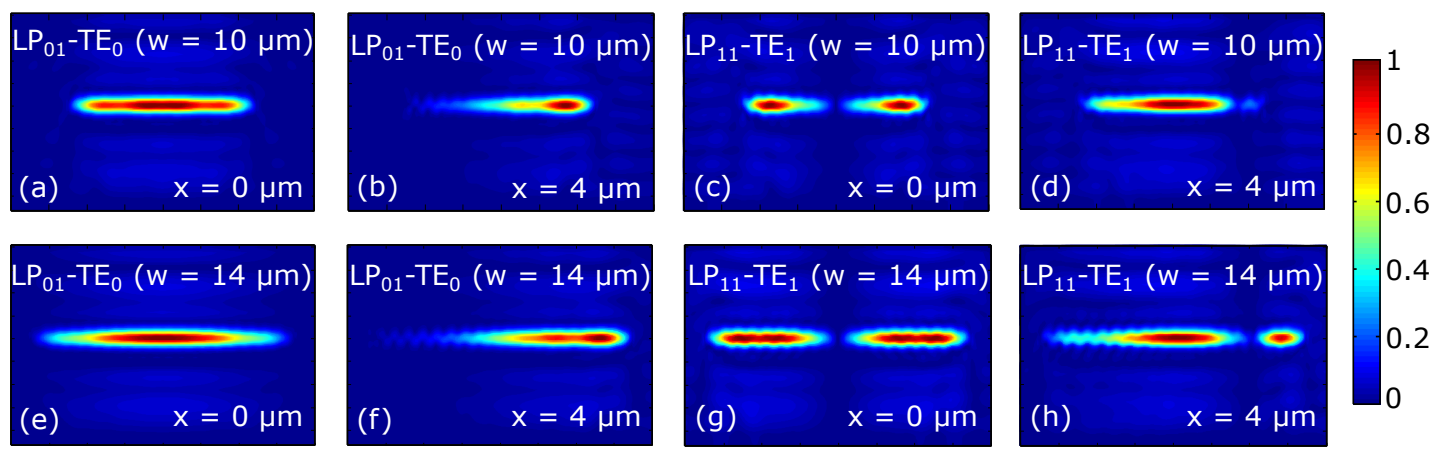

Figure 4.3.8. Normalized optical intensity in the SOI waveguide (width $w_{g}$, height $220 \mathrm{~nm}$ ) when the grating coupler is excited from a TMF fiber for different coupler widths ( $w_{g}=10 \mu \mathrm{m}$ and $\left.w_{g}=14 \mu \mathrm{m}\right)$, fiber-to-coupler misalignment $(x=0 \mu m$ and $x=4 \mu \mathrm{m})$ and exciting fiber mode ( $\left.L P_{01}, L P_{11}\right)$. 
Figure 4.3.8 shows the optical intensity profiles at the grating coupler output (at a $\Delta z=15 \mu \mathrm{m}$ distance from the end of the grating coupler) when the coupler is excited from a TMF fiber with just one mode with the proper polarization and azimuthal orientation. These plots are presented for the most sensitive design and the selected width from Figure 4.3.7, that is, $w_{g}=10 \mu \mathrm{m}(\mathrm{a}-\mathrm{d})$ and $w_{g}=14 \mu \mathrm{m}$ (e-h) waveguide widths. Two positions for the fiber are shown in the figure, centered $(x=0 \mu \mathrm{m})$ and with a $4 \mu \mathrm{m}$ lateral misalignment. The field intensity is represented by color scale, being brown the most intense area and blue the least one.

$\mathrm{TE}_{0}$ is symmetric at $x=0 \mu \mathrm{m}$ and non-symmetric at $x=4 \mu \mathrm{m}$ respect to $x-y$ axes for both $w_{g}=10 \mu \mathrm{m}(\mathrm{a}-\mathrm{b})$ and $w_{g}=14 \mu \mathrm{m}$ (e-f) waveguides. Without displacement, mode profiles exhibit a unique Gaussian profile whereas for a $4 \mu \mathrm{m}$ displacement, a fraction of the $\mathrm{TE}_{0}$ power is converted into $\mathrm{TE}_{1}$ mode. That trend is more pronounced as we increase the waveguide width. Apart from that, for $w_{g}=14 \mu \mathrm{m}$, the mode profile is more confined than for the other width at $x=0 \mu \mathrm{m}$ and less confined at $x=4 \mu \mathrm{m}$, which is consistent with the coupling efficiency results.

$\mathrm{TE}_{1}$ is symmetric at $x=0 \mu \mathrm{m}$ and breaks the symmetry at $x=4 \mu \mathrm{m}$ respect to $x-y$ axes for both $10 \mu \mathrm{m}(\mathrm{c}-\mathrm{d})$ and $14 \mu \mathrm{m}(\mathrm{g}-\mathrm{h})$ waveguides. Undesired mode conversion is evidenced for a $4 \mu \mathrm{m}$ displacement by the conversion of $\mathrm{TE}_{1}$ mode into $\mathrm{TE}_{0}$. This effect is enhanced for the $w_{g}=10 \mu \mathrm{m}$ waveguide in which case most of the power has been transformed into $\mathrm{TE}_{0}$ mode (Figure 4.3.8(d)). $\mathrm{LP}_{11}$ is coupled to the $\mathrm{TE}_{1}$ mode for both waveguide widths, nonetheless, the original mode profile is better preserved for the $w_{g}=14 \mu \mathrm{m}$ waveguide.

\section{EXPERIMENTAL RESULTS}

The grating couplers with $12 \mu \mathrm{m}$ and $14 \mu \mathrm{m}$ waveguide widths have been fabricated on samples from 6" SOI wafers. The wafers were purchased from SOITEC and have a $220 \mathrm{~nm}$ thin silicon top layer and a $2 \mu \mathrm{m}$ thick buried oxide layer. The total wafer thickness is approximately $750 \mu \mathrm{m}$, taking into account the silicon bulk. The NTC laboratory employed the SOI CMOS compatible process based on an electron beam direct writing lithography on HSQ resist, followed by dry etching of $220 \mathrm{~nm}$ SOI wafers, a typical process to be found in fabrication foundries.

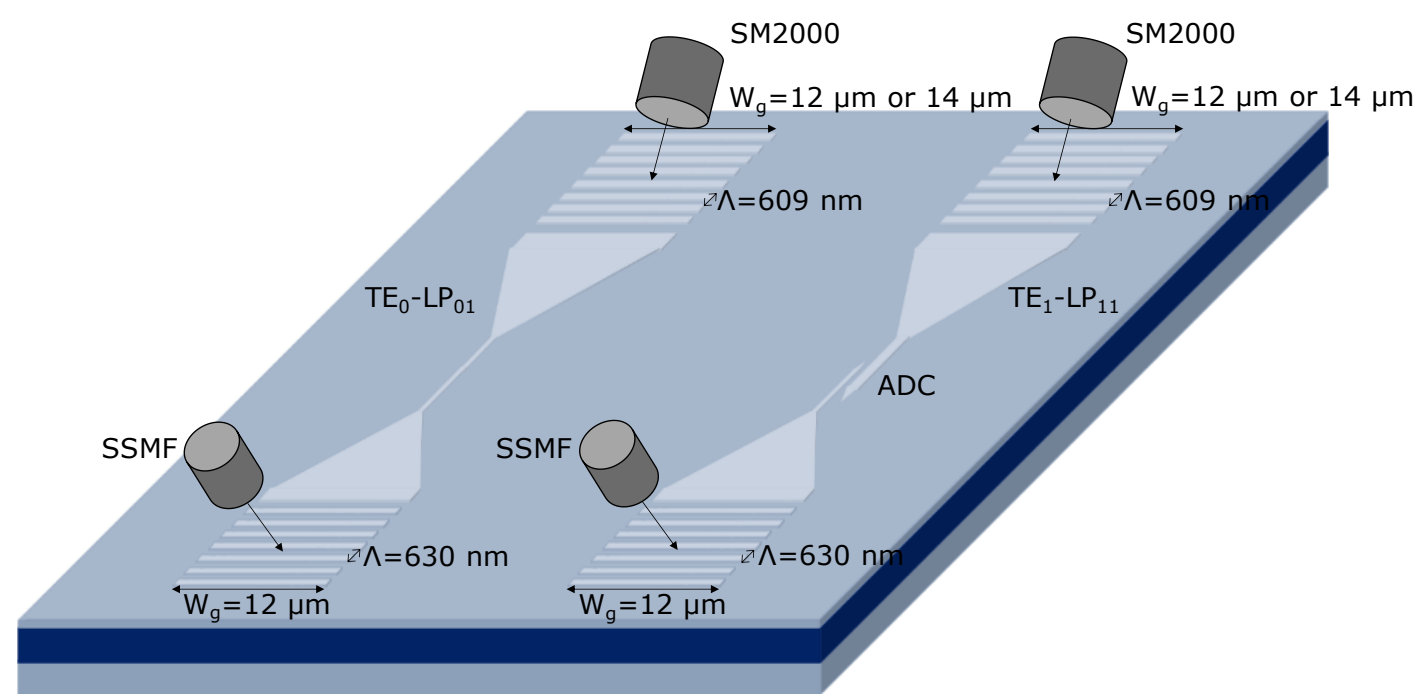

Figure 4.3.9. $3 D$-sketch of the experimental configuration in order to achieve the $L P_{01}$ and $L P_{11}$ modes in the TMF.

Figure 4.3.9 shows the experimental configuration based on two schemes, one for the $\mathrm{TE}_{0}-\mathrm{LP}_{01}$ mode coupling and the other for the $T E_{1}-L_{11}$. In both cases, light is launched to a standard $\mathrm{LP}_{01}-\mathrm{TE}_{0}$ grating $\left(w_{g}=12 \mu \mathrm{m}\right)$ by means of a standard single-mode fiber (SSMF). Previously, a polarization controller is employed to adjust and maximize the coupling to the grating coupler. When the first scheme is considered, the configuration does not need an ADC as only TE mode $_{0}$ is propagated and coupled to the $\mathrm{LP}_{01}$ mode in the TMF. The second scheme uses a mode converter based on an asymmetrical directional coupler (ADC) in order to transform the $\mathrm{TE}_{0}$ 
into $\mathrm{TE}_{1}[6]$. Next, the $\mathrm{TE}_{1}$ mode reaches the output grating coupler and it is finally coupled to the TMF. The optical modes are propagated in the TMF pigtail with a length of 2 meters. Additionally, to avoid any undesirable reflection, the ADC waveguides were tapered.

In order to characterize the performance of the grating couplers $\left(w_{g}=12 \mu \mathrm{m}\right.$ and $w_{g}=14 \mu \mathrm{m}$ wide) the insertion losses and the modal purity of the $\mathrm{LP}_{01}$ and $L \mathrm{P}_{11}$ modes in the TMF output were measured for all four cases. The modal purity has been determined by means of the normalized mode overlap integral of the measured mode with the theoretical pure $L P_{01}$ or $L P_{11}$ mode for the SM2000 fiber.
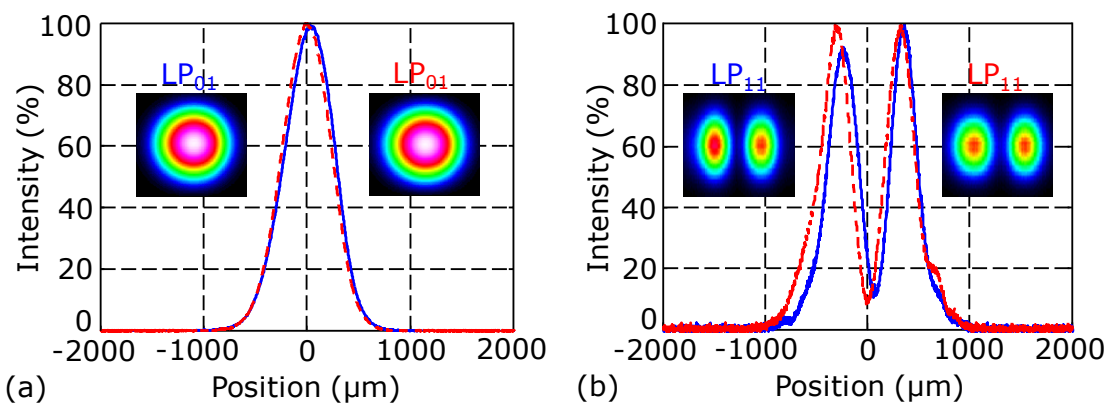

Figure 4.3.10. Experimental intensity pattern and modal profile at the TMF from $12 \mu \mathrm{m}$ (solid blue line) and $14 \mu \mathrm{m}$ (dashed red line) waveguide widths for: (a) $L P_{01}$ mode and (b) $L P_{11}$ mode.

Figure 4.3.10 depicts the intensity pattern and modal profile of the $L P_{01}$ and $L P_{11}$ modes when the grating coupler is driven by the $\mathrm{TE}_{0}$ or the $\mathrm{TE}_{1}$ modes to be coupled to the SM2000 TMF. A CCD camera Beam Profiler (Thorlabs BP209-IR) is placed at the output of the TMF verifying the correct coupling of both modes for the $12 \mu \mathrm{m}$ and $14 \mu \mathrm{m}$ widths. Both $\mathrm{LP} \mathrm{P}_{11}$ modes achieved a perfect Gaussian profile (modal purity of $100 \%$ ) and the two $\mathrm{LP}_{11}$ modes obtained a modal purity of $89 \%$ for the $w_{g}=12 \mu \mathrm{m}$ width and $92 \%$ for the $w_{g}=14 \mu \mathrm{m}$ width, respectively. The insertion losses (IL) for the $L P_{01}$ mode were $9 \mathrm{~dB}$ and $8.2 \mathrm{~dB}$, respectively. In the case of the $\mathrm{LP}_{11}$ mode, the IL obtained were $18 \mathrm{~dB}$ and $16 \mathrm{~dB}$, respectively. It can be stated that the $\mathrm{LP}_{01}$ mode achieved a better power coupling than $\mathrm{LP}_{11}$ mode due to the extra losses added by the mode integrated mode converter needed to obtain the $\mathrm{TE}_{1}$ mode in the experimental setup. These insertion losses values correspond to the losses from the laser output to the TMF output including all fiber pigtails, connectors and polarization controller needed for the experiment. Finally, both modes obtained an excellent modal purity and the insertion losses are lower than the state of the art result for $\mathrm{TE}_{1}-\mathrm{LP}_{11}$ vertical grating couplers in SOI technology which show insertion losses higher than $20 \mathrm{~dB}$ [21-23].
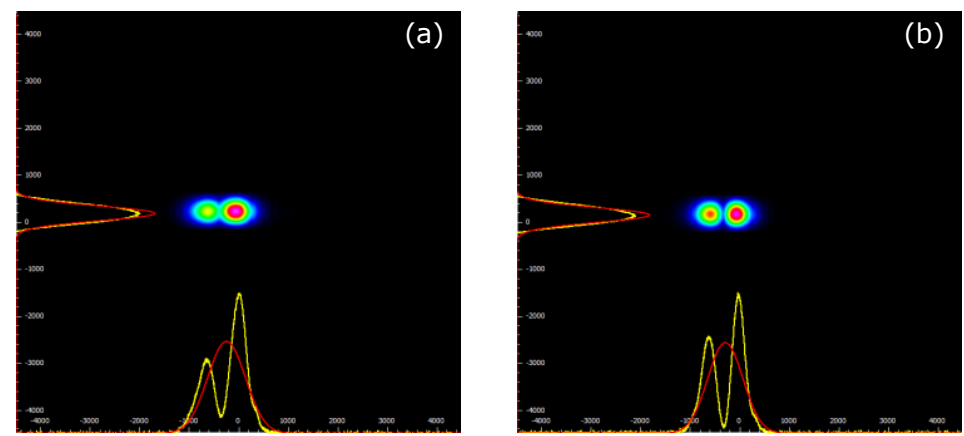

Figure 4.3.11. Captured image of the intensity pattern and modal profile of the $L P_{11}$ modes when a little misalignment in the coupling from fiber to grating coupler is produced for: (a) $12 \mu \mathrm{m}$ width and (b) $14 \mu \mathrm{m}$ width

In order to check the sensitivity of both designs to the fiber to grating coupler misalignment, the fiber was slightly laterally misplaced when the $T E_{1}$ to $L P_{11}$ was under measurement and the results are shown in Figure 4.3.11. The pictures were captured moving the positioner (NEWPORT M-562 XYZ with DM 13 micrometers) around $3 \mu \mathrm{m}$ respect to the waveguide center. Thus, the LP 11 mode profile at the SM2000 fiber output for the $w_{g}=14 \mu \mathrm{m}$ coupler obtains a higher purity $(86 \%)$ than the $\mathrm{LP}_{11}$ mode for the $w_{g}=12 \mu \mathrm{m}$ coupler $(77 \%)$, as shown in 
Figure 4.3.11. The experimental results confirm the greater tolerance to fiber coupler lateral misalignments for the $w_{g}=14 \mu \mathrm{m}$ design.

\section{Conclusion}

In this paper, we have reported the simulation and experimental analysis of the $\mathrm{LP}_{01}$ and $\mathrm{LP}_{11}$ fiber modes coupling to the $\mathrm{TE}_{0}$ and $\mathrm{TE}_{1}$ SOI waveguide modes through grating couplers. Different grating coupler widths $(10 \mu \mathrm{m}$ to $15 \mu \mathrm{m})$ have been assessed in terms of $\mathrm{LP}_{01}-\mathrm{TE}_{0}$ and $\mathrm{LP}_{11}-\mathrm{TE}_{1}$ coupling efficiency, sensitivity to lateral misalignment between the coupler and the fiber and design compactness. The optimum design $\left(\Lambda=609 \mathrm{~nm}, e_{d}=70 \mathrm{~nm}, f f=0.5\right)$ obtained a $49 \% \mathrm{LP}_{01}-\mathrm{TE}_{0}$ efficiency with a $5 \mu \mathrm{m}$ alignment tolerance whereas the $\mathrm{LP}_{11}-\mathrm{TE}_{1}$ efficiency is $45 \%$ with a $2.6 \mu \mathrm{m}$ alignment tolerance. Both efficiency results are quite close to the theoretical maximum efficiency of $50 \%$. The coupling efficiency and the sensitivity to lateral misalignment have been experimentally assessed for two different widths, $14 \mu \mathrm{m}$ as optimum design and $12 \mu \mathrm{m}$ as the typical width for single mode $\mathrm{TE}_{0}-\mathrm{LP}_{01}$ couplers. The near field pattern at the two mode fiber output confirms the perfect coupling of both modes for the $14 \mu \mathrm{m}$ width with a modal purity of $100 \%$ and $90 \%$, respectively.

\section{References}

[1] D. J. Richardson, "New optical fibres for high-capacity optical communications," Philos. Trans. R. Soc. Lond. A 374(2062), 20140441 (2016).

[2] M. Kasahara, K. Saitoh, T. Sakamoto, N. Hanzawa, T. Matsui, K. Tsujikawa, F. Yamamoto, and M. Koshiba, "Design of few-mode fibers for mode-division multiplexing transmission," IEEE Photonics J. 5(6), 7201207 (2013).

[3] H. Chen, V. Sleiffer, B. Snyder, M. Kuschnerov, R. Uden, Y. Jung, C. M. Okonkwo, O. Raz, P. O'Brien, H. Waardt, and T. Koonen, "Demonstration of a photonic integrated mode coupler with MDM and WDM transmission," Photon. Technol. Lett. 25(21), 2039-2042 (2013).

[4] C. Sun, Y. Yu, M. Ye, G. Chen, and X. Zhang, "An ultra-low crosstalk and broadband two-mode (de)multiplexer based on adiabatic couplers," Sci. Rep. 6, 38494 (2016).

[5] J. Xing, Z. Li, X. Xiao, J. Yu, and Y. Yu, "Two-mode multiplexer and demultiplexer based on adiabatic couplers," Opt. Lett. 38(17), 3468-3470 (2013).

[6] D. Garcia-Rodriguez, J. L. Corral, A. Griol, and R. Llorente, "Dimensional variation tolerant mode converter/multiplexer fabricated in SOI technology for two-mode transmission at 1550 nm," Opt. Lett. 42(7), 1221-1224 (2017).

[7] D. Garcia-Rodriguez, J. L. Corral, and R. Llorente, "Mode Conversion for Mode Division Multiplexing at $850 \mathrm{~nm}$ in Standard SMF," Photon. Technol. Lett. 29(11), 929-932 (2017).

[8] Z. Zhang, X. Hu, and J. Wang, "On-chip optical mode exchange using tapered directional coupler," Sci. Rep. 5, 16072 (2015).

[9] Y. Ding, J. Xu, F. Da Ros, B. Huang, H. Ou, and C. Peucheret, "On-chip two-mode division multiplexing using tapered directional coupler-based mode multiplexer and demultiplexer," Opt. Express 21(8), 10376-10382 (2013).

[10] A. Zanzi, A. Brimont, A. Griol, P. Sanchis, and J. Marti, "Compact and low-loss asymmetrical multimode interference splitter for power monitoring applications," Opt. Lett. 41(2), 227-229 (2016).

[11] T. Uematsu, K. Saitoh, N. Hanzawa, T. Sakamoto, T. Matsui, K. Tsujikawa, and M. Koshiba, "Low-loss and broadband PLC-type mode (de)multiplexer for mode-division multiplexing transmission," OFC2013, paper OTh1B.5 (2013).

[12] N. Hanzawa, K. Saitoh, T. Sakamoto, T. Matsui, K. Tsujikawa, M. Koshiba, and F. Yamamoto, "Two-mode PLC-based mode multi/demultiplexer for mode and wavelength division multiplexed transmission," Opt. Express 21(22), 25752-25760 (2013). 
[13] Y. Lai, Y. Yu, S. Fu, J. Xu, P.P. Shum, and X. Zhang, "Efficient spot size converter for higher order mode fiber-chip coupling," Optics Letters 42(18), 3702-3705 (2017).

[14] Nobuaki Hatori, Takanori Shimizu, Makoto Okano, Masashige Ishizaka, Tsuyoshi Yamamoto, Yutaka Urino, Masahiko Mori, Takahiro Nakamura, and Yasuhiko Arakawa, "A Hybrid Integrated Light Source on a Silicon Platform Using a Trident Spot-Size Converter," J. Lightwave Technol. 32(7), 1329-1336 (2014).

[15] N. Hanzawa, K. Saitoh, T. Sakamoto, T. Matsui, K. Tsujikawa, M. Koshiba, and F. Yamamoto, "Mode multi/demultiplexing with parallel waveguide for mode division multiplexed transmission," Opt. Express 22(24), 29321-29330 (2014).

[16] D. Dai and M. Mao, "Mode converter based on an inverse taper for multimode silicon nanophotonic integrated circuits," Opt. Express 23(22), 28376-28388 (2015).

[17] D. Taillaert, F. Van Laere, M. Ayre, W. Bogaerts, D. Van Thourhout, P. Bienstman, and R. Baets, "Grating Couplers for Coupling between Optical Fibers and Nanophotonic Waveguides," Jpn. J. Appl. Phys. 45, 6071-6077 (2006).

[18] S. Lardenois, D. Pascal, L. Vivien, E. Cassan, S. Laval, R. Orobtchouk, M. Heitzmann, N. Bouzaida, and L. Mollard, "Low-loss submicrometer silicon-on-insulator rib waveguides and corner mirrors," Opt. Lett. 28, 1150-1152 (2003).

[19] R. Orobtchouk, A. Layadi, H. Gualous, D. Pascal, A. Koster, and S. Laval, "High-efficiency light coupling in a submicrometric silicon-on-insulator waveguide," Appl. Opt. 39(31), 57735777 (2000).

[20] Y. Ding, H. Ou, J. Xu, M. Xiong, and C. Peucheret, "On-chip mode multiplexer based on a single grating coupler" IEEE Photonics Conference IPC (IEEE, 2012), paper ThB4.

[21] B. Wohlfeil, G. Rademacher, C. Stamatiadis, K. Voigt, L. Zimmermann, and K. Petermann, "A Two-Dimensional Fiber Grating Coupler on SOI for Mode Division Multiplexing," Photon. Technol. Lett. 28(11), 1241-1244 (2016).

[22] A. M. J. Koonen, H. Chen, H. P. A. van den Boom, and O. Raz, "Silicon photonic integrated mode multiplexer and demultiplexer," IEEE Photon. Technol. Lett. 24(21), 1961-1964 (2012).

[23] Y. Ding, H. Ou, J. Xu, C. Peucheret, "Silicon photonic integrated circuit mode multiplexer", Photon. Technol. Lett. 25(7), 648-651 (2013).

[24] J. V. Galan, P. Sanchis, J. Blasco, and J. Marti, "Study of High Efficiency Grating Couplers for Silicon-Based Horizontal Slot Waveguides," IEEE Photon. Technol. Lett. 20(12), 985-987 (2008).

[25] G. Roelkens, D. Van Thourhout, and R. Baets, "High efficiency Silicon-on-Insulator grating coupler based on a poly-Silicon overlay," Opt. Express 14, 11622 (2006).

[26] D. Taillaert, "Grating couplers as Interface between Optical Fibres and Nanophotonic Waveguides," PhD Dissertation, Universiteit Gent, 2005. 


\section{ChAPTER 5. General Discussion}

The articles presented in the Chapters 3 and 4 constitute the core of the research done in this Ph.D. Thesis. These papers cover two different scenarios concerning the mode division multiplexing. Basically, the contents of the articles can be summarized in six different topics that correspond to the following sections in which this Chapter has been splitted, namely: fused optical coupler (section 5.1), Mechanical mode converter (section 5.2), Commercial optical couplers (section 5.3), Strip waveguide (section 5.4), Supermodes based on strip and ridge waveguides (section 5.5), and bimodal grating coupler (section 5.6).

This chapter will give an overview of the aforementioned articles, detailing the motivation, objectives, main results and conclusions for each analyzed problem.

\subsection{Fused optical coupler}

It is well-known that the main multiplexers/demultiplexers are based on optical couplers that are the key components in a MDM system to combine and split the different modulated optical signals. In fact, recent studies reveal the use of fiber based directional couplers for a MDM system at $1550 \mathrm{~nm}$ [LI12], [ISM14], [Li13]. However, the system relies on the use of a specialty fiber, increasing the cost of the system and not taking profit of the actual SSMF deployment. Thus, it is proposed another technique to avoid this problem. On the one hand, the use of the SSMF opens up the possibility to employ the already deployed fiber as a twomode fiber (TMF) if short wavelengths below cutoff wavelength are chosen. On the other hand, the use of VCSELs with modulation bandwidth beyond $10 \mathrm{~Gb} / \mathrm{s}$ at $850 \mathrm{~nm}$ offers a low cost device.

In this section, the feasibility of using fused optical couplers at $850 \mathrm{~nm}$ wavelength with the symmetric and asymmetric structures is explored. To reach this aim, in a first stage it is analyzed the structure of the coupler taking into account the coupled mode theory. The coupling between modes is conditioned by the coupling coefficients which are related with the physical parameters of the structure, the separation between cores and the coupling length. In the symmetric structure, the phase matching condition is always fulfilled since the optical modes to couple are the same. However, the asymmetric structure needs to select properly the features of the fiber (reducing its core) in order to achieve the same effective indexes of the modes that will be coupled. In a second stage, both structures were simulated considering different designs according to the separation between cores and coupling length. The results obtained revealed that the asymmetric coupler reduces the core diameter of the TMF to $4.17 \mu \mathrm{m}$ ( $51 \%$ of its original core diameter) in order to fulfill the phase matching condition between the $L P_{01}$ mode from the single mode fiber and the $L P_{11}$ mode in the TMF. In this case, the coupling efficiency and the insertion losses determine the optimum design. Therefore, if the coupler acts a mode converter or multiplexer, the configuration with the lower spacing and the shorter coupling length $\left(d=6 \mu \mathrm{m}, L_{1}=15.66 \mathrm{~mm}\right.$ ) achieved a $93.5 \%$ (corresponding to a $0.3 \mathrm{~dB}$ insertion loss) coupling efficiency for a $10 \mathrm{~nm}$ bandwidth around $850 \mathrm{~nm}$. Also, when the coupler acts as a mode conversion or demultiplexer the best configuration is again achieved for the lower spacing and the shorter coupling length with an extinction ratio above $11.6 \mathrm{~dB}$ and a leakage above $33.6 \mathrm{~dB}$. However, the performance as demultiplexer is limited by the sensitivity of the $\mathrm{LP}_{11}$ mode and, therefore, it would be improved by adding a modal filter after the TMF output of the coupler with $20 \mathrm{~dB}$ attenuation for 30 turns around a cylinder of 9-mm radius [MOO05]. In the symmetric coupler case, the results revealed that the phase matching condition is always fulfilled since the modes are the same, however, a mode converter is needed in the multiplexer in order to convert the $L P_{01}$ mode into $L P_{11}$ mode. However, this limitation is balanced due to that is not required to employ a mode converter in the demultiplexer. When the minimum coupling length for any fixed fiber separation is selected, the coupling efficiency and the insertion losses are quite similar for all configurations (higher than $99 \%$ with insertion losses around $0.09 \mathrm{~dB}$ in a $10 \mathrm{~nm}$ bandwidth). However, if the demultiplexer is considered, the best overall performance is achieved for the wider separation 
( $d=8 \mu \mathrm{m}, L=56.58 \mathrm{~mm}$ ), taking into account the same proposed consideration in the asymmetrical structure when the demultiplexer was considered.

Finally, in both structures the coupling efficiency and the extinction ratio were maximized for a separation between fibers and a coupling length of $d=6 \mu \mathrm{m}, L_{1}=15.66 \mathrm{~mm}$ and $d=8 \mu \mathrm{m}$, $L=56.58 \mathrm{~mm}$, respectively. Also, it is worth to mention that a polarization controller is needed after the TMF in order to tune the orientation of the optical mode according to the plane containing the fibers of the coupler.

\subsection{Mechanical mode converter}

The mode converters and mode (de)multiplexers are required to combine and split the modes. Several techniques such as those based on spatial light modulators or q-plates have been reported [MIL15], However, the devices based on fused fibers offer a compact solution, reducing the losses and with direct integration with the optical fiber. For example, the symmetrical and asymmetrical couplers are good alternatives to the spatial light modulators. In the case of the asymmetrical couplers, the fabrication tolerances required by the asymmetric couplers are more difficult to fulfill than the equivalent tolerances for the symmetric device but, as a counterpart, the symmetrical couplers previously need a mode converter in order to convert the $L P_{01}$ mode into $L P_{11}$ mode. The use of a mode converter based on a mechanical grating at $1550 \mathrm{~nm}$ has been reported [GIL12], [PRA15]. Nevertheless, the main drawback is the use of a special fiber which increases the system cost. An alternative is to employ the SMF28 at $850 \mathrm{~nm}$, taking advantage of the benefits explained in the section 5.1.

In this section, the design and fabrication of a mechanical grating at $850 \mathrm{~nm}$ band is exhaustively analyzed. Firstly, the mode conversion is created causing a periodic perturbation on the optical fiber through a grating period with a certain number of coupling points. The refractive effective indexes are the key of the mode conversion in order to determine the grating period and the detuning parameter. Furthermore, the effective grating period can be tuned in a wide bandwidth due to the relative inclination of the grating axis with respect to the fiber $\left(0^{\circ}\right.$ to $\left.15^{\circ}\right)$. This variation in the effective grating period adds flexibility to adjust the mode converter to the both the specific characteristics of the fiber and the wavelength emitted by the laser. This variation in the grating axis allows tuning the effective grating period in a $124 \mathrm{~nm}$ bandwidth (from $798 \mathrm{~nm}$ to $922 \mathrm{~nm}$ ). Then, an experimental analysis transmitting the $\mathrm{LP}_{01}$ and $\mathrm{LP}_{11}$ modes with $1.25 \mathrm{~Gb} / \mathrm{s}$ and $2.5 \mathrm{~Gb} / \mathrm{s}$ along $1 \mathrm{~km}$ length fiber was realized. In a first step, the $\mathrm{LP}_{11}$ mode output is erased using a mode striper based on a cylinder of $1 \mathrm{~cm}$ diameter, obtained by bending the fiber in a few loops without damaging the $\mathrm{LP}_{01}$ mode (losses $<0.01 \mathrm{~dB}$ ). In a second step, the pure $L P_{01}$ mode is converted into $\mathrm{LP}_{11}$ mode using the mechanical grating applying pressure over the fiber (losses around 0.2-0.5 dB), with a $250 \mu \mathrm{m}$ cladding and no additional coating. It must be taken into account that if the pressure is too high the fiber will be broken. For the optimum mode converter configuration these losses are equal to $0.5 \mathrm{~dB}$, achieving a mode conversion around $89 \%$ (losses of $9.5 \mathrm{~dB}$ ). After that, each mode ( $L P_{01}$ or $L P_{11}$ ) is transmitted over $1 \mathrm{~km}$ using $1.25 \mathrm{~Gb} / \mathrm{s}$ and $2.5 \mathrm{~Gb} / \mathrm{s}$ employing an OOK signal with a $2^{31}-1$ pseudo random bit sequence (PRBS). This transmission was analyzed taking into account the quality factor $(Q)$ that it is directly related to the bit error rate $(B E R)$. The results revealed that the transmissions for both modes over $1.25 \mathrm{~Gb} / \mathrm{s}$ and $2.5 \mathrm{~Gb} / \mathrm{s}$ obtained a quality factors around 6.8 and 6.2, respectively. It is important to mention that both signals have a quality factor higher than $Q=6$, corresponding to a $B E R=10^{-9}$.

Finally, the mechanical grating was designed to operate in a $124 \mathrm{~nm}$ bandwidth allowing converting the $\mathrm{LP}_{01}$ mode into $\mathrm{LP}_{11}$ with a $89 \%$ conversion efficiency. The data transmission over $1 \mathrm{~km}$ at $1.25 \mathrm{~Gb} / \mathrm{s}$ and $2.5 \mathrm{~Gb} / \mathrm{s}$ OOK signal was achieved obtaining a quality factor higher than $Q=6\left(B E R=10^{-9}\right)$ for both modes. The link distance and achieved bitrate were limited by the reduced output power $(-13 \mathrm{dBm})$ and modulation bandwidth of the VCSEL source. 


\subsection{Commercial optical couplers}

As it was discussed in sections 5.1 and 5.2, mode converters and multiplexers/demultiplexers to combine/divide the different modulated signals are still the key devices for the implementation of MDM links. The most typical applications are based on a specialty fiber at $1550 \mathrm{~nm}$ wavelength. However, with the aim to improve the techniques proposed in the last sections, the use of commercially-available standard single-mode couplers designed for the $1550 \mathrm{~nm}$ window operating at $850 \mathrm{~nm}$ represent an optimum and low cost solution. In fact, the design of a new fiber (symmetrical or asymmetrical structures) or the use of a mechanical mode converter is not required. This opens up the possibility to simplify the MDM system reducing the insertion losses.

This section covers the commercial optical couplers analysis for the $L P_{01}$ and $L P_{11}$ modes coupling. Several types of commercial couplers with distinct coupling ratios are considered. In a first stage, the mode coupling theory is studied. The simulation results were performed using the commercial software RSoft 3D-BPM. This tool is able to analyze the coupler structure taking into account the coupling length and the separation between cores. The results obtained both from theory and simulation revealed that different coupling ratios are useful at $850 \mathrm{~nm}$. For a coupling length of $17 \mathrm{~mm}$ and separation between cores of $6 \mu \mathrm{m}$, the $L P_{01}$ and $L P_{11}$ modes obtained a coupling efficiency of $98 \%$ and $100 \%$, respectively. In a second stage, it was explored the demultiplexing of the $\mathrm{LP}_{01}$ and $\mathrm{LP}_{11}$ modes taking into account the different coupling ratios. In this case, all couplers achieved a high pure mode for both orthogonal polarizations ( $\mathrm{LP}_{11 \mathrm{a}}$ and $\left.\mathrm{LP}_{11 \mathrm{~b}}\right)$. However, only the 90:10 optical coupler obtained a similar behavior at $850 \mathrm{~nm}$ than the specs at $1550 \mathrm{~nm}$. Fortunately, all couplers obtained purities higher than $90 \%$, thus demonstrating an excellent purity after the demultiplexing process.

Finally, the commercial optical coupler at $850 \mathrm{~nm}$ operation confirms the correct demultiplexing of the optical modes, specifically the 90:10 optical coupler. The simulation results indicate a coupling efficiency of $98 \%$ and $100 \%$ for the $L P_{01}$ and $L P_{11}$ modes at $850 \mathrm{~nm}$, respectively. The experimental results achieved a purity of $100 \%$ and $90 \%$ for the $\mathrm{LP}_{01}$ and $\mathrm{LP}_{11}$ modes, respectively.

\subsection{Strip waveguide}

Mode converters and mode (de)multiplexers, based on integrated technology (silicon-oninsulator (SOI) or silica-based planar lightwave circuit (PLC)), have been extensively analyzed. Besides, many studies about asymmetrical directional couplers (ADCs) have focused on the conversion of the $\mathrm{TE}_{0}$ mode to $\mathrm{TE}_{1}$ mode, or higher modes [WAN14], [DIN13]. However, the $A D C$ optimization taking into account the fabrication tolerances is a topic that has not been covered yet. In fact, the ADCs proposed are quite sensitive to dimensional variations, and excess losses around $2 \mathrm{~dB}$ or crosstalk levels up to $10 \mathrm{~dB}$ must be accepted for size deviations up to $\pm 10 \mathrm{~nm}$. Unfortunately, the $10 \mathrm{~nm}$ are not uncommon in typical SOI technology [XU14], which would lead to low device yields or high thermal tuning powers.

In this section, it is carried out a comprehensive study of the optimum dimensions for an ADC mode converter and (de)multiplexer in SOI technology. In a first stage, the structure of the ADC was analyzed taking into account the coupled mode theory. The mode conversion of the $T E_{0}$ into $T E_{1}$ mode depends on the coupling length and separation between waveguides whenever that the phase mismatch condition is fulfilled $(\delta=0)$. In that case, both effective refractive indexes will be equal, however, in the ADCs the effective indices must be matched by properly selecting the dimensions of the respective waveguides. In a second stage, the device was simulated considering different designs according to the separation between cores and coupling lengths. In fact, the coupling efficiency and the insertion losses were analysed, obtaining an evaluation of the performance of the ADC around $1550 \mathrm{~nm}$ and its robustness against fabrication tolerances. The best configuration was obtained for the $0.2 \mu \mathrm{m}$ gap $(L=30.74 \mu \mathrm{m}$ ) with a $98.7 \%$ coupling efficiency (corresponding to $0.06 \mathrm{~dB}$ excess loss). However, for the $0.1 \mu \mathrm{m}$ gap $(L=12.50 \mu \mathrm{m})$ case an extinction ratio better than $24.2 \mathrm{~dB}$ is achieved in a $20 \mathrm{~nm}$ range around $1550 \mathrm{~nm}$. In a third stage, the parameters previously 
selected were analyzed taking into account the waveguide widths variations. The results obtained from simulation revealed that the lowest gap $(0.1 \mu \mathrm{m})$ gets the best robust design to fabrication tolerances. This statement was also confirmed by experimental measurements, demonstrating the coupling efficiency obtained (90\%) for a $0.1 \mu \mathrm{m}$ gap at $1550 \mathrm{~nm}, 7.5 \%$ below the theoretical coupling, corresponding to an additional $0.35 \mathrm{~dB}$ excess loss. The small differences is due to the fabrication tolerances, however, if the same error had occurred in the $0.2 \mu \mathrm{m}$ and $0.3 \mu \mathrm{m}$ gap devices, the efficiency would be around $60 \%$ and $20 \%$, respectively.

Finally, the ADC obtained a mode conversion and mode (de)multiplexing of $97 \%$ efficiency and a $23.4 \mathrm{~dB}$ crosstalk level in the $1540-1560 \mathrm{~nm}$ band using a $0.1 \mu \mathrm{m}$ gap. Also, it was achieved a coupling efficiency higher than $82.3 \%$ (corresponding to $0.8 \mathrm{~dB}$ excess loss) with $3 \sigma$ accuracy. Regarding with the experimental results, it was found a $90 \%$ coupling efficiency.

\subsection{Supermodes based on strip and ridge waveguides}

Most of the mode converters and (de)multiplexers based on silicon-on-insulator (SOI) or planar lightwave circuit (PLC) processing technologies are cost-effective optical devices such as asymmetrical directional couplers (ADCs) [GAR17]. However, the SOI technology has been appointed as a promising technology to integrate several functions due to the integration requirements [WAN14]. In fact, the ADC devices based on SOI technology with strip structure offer an excellent yield, although due to the high phase matching requirements they are inherently sensitive to fabrication errors [GAR17]. Thus, a new method of analysis, using the effective refractive indexes of the even and odd supermodes, has been recently used to study the performance and structure of different integrated devices based on coupling waveguides [PAN16], [MIK14]. The implementation of this method in strip and ridge structures for directional couplers (DC) demonstrated that the DC ridge design is four times more robust than DC strip design [MIK14].

In this section, the analysis of the sensitivity of the coupling efficiency to variations on the width, height, gap, or slab thickness dimensions has been carried out for both strip and ridge ADCs. In a first stage, the coupled mode theory based on the use of the supermodes determines the sensitivity of the coupling efficiency to fabrication tolerances. In a second step, several designs (gaps from $100 \mathrm{~nm}$ to $400 \mathrm{~nm}$ and waveguide widths for the fundamental mode from $w_{A}=430 \mathrm{~nm}$ to $470 \mathrm{~nm}$ ) have been selected. In order to fulfill the phase matched condition, the width of the waveguide for the first high order mode was properly selected. The results showed an optimum robust design for the $100 \mathrm{~nm}$ gap, both for simultaneous gap, width and height variations. However, in the third step, the ridge structure was analyzed taking into account the same characteristics but adding the slab thickness parameter. In this case, the results obtained different robust designs for different gaps: the $400 \mathrm{~nm}$ gap considering the anti-correlated changes in width and gap $\left(0.14 \cdot 10^{-3} \mathrm{~nm}^{-1}\right)$, the $100 \mathrm{~nm}$ gap for changes in height $\left(-0.1 \cdot 10^{-3} \mathrm{~nm}^{-1}\right)$, and $300 \mathrm{~nm}$ gap (when $w_{A}$ is $450 \mathrm{~nm}$ ) for changes in the slab thickness $\left(0.7 \cdot 10^{-3} \mathrm{~nm}^{-1}\right)$. The difference in the sensitivity parameter is determined by a factor of $6\left(0.14 \cdot 10^{-3} \mathrm{~nm}^{-1}\right.$ by $-0.074 \cdot 10^{-2} \mathrm{~nm}^{-1}$, for the ridge and strip structures, respectively).

Finally, the ADC obtained an optimum gap design (100 nm and $400 \mathrm{~nm}$ ) for strip and ridge structures, taking into account the supermode analysis based on width, height, gap and slab thickness variations produced with respect to the nominal design.

\subsection{Bimodal grating coupler}

Grating couplers have been extensively analyzed in the case of $\mathrm{TE}_{0}-\mathrm{LP}_{01}$ mode coupling from the optical waveguide to the single-mode standard fiber (SSMF) or vice versa. Besides, many studies have focused their attention on the study of increasing the coupling efficiency with apodized gratings or bottom reflectors [ANT11], [VAN07]. However, the grating coupler using the first high order mode is a topic not covered so far. In fact, very little literature about the coupling of the first high order mode using grating couplers can be found [WOH16], [DIN13]. Also, these references are based on the use of multiple fundamental modes to generate the first high order mode in the optical fiber. 
In this section, it is analyzed the coupling of the $\mathrm{TE}_{0}$ and $\mathrm{TE}_{1}$ modes into one single polarization of the $L P_{01}$ mode and one single polarization and azimuthal orientation of the $\mathrm{LP}_{11}$ mode of the two mode fiber (TMF) at $1550 \mathrm{~nm}$. The coupling to/from grating couplers is defined by the Bragg condition, relating the wave-vectors of the incident and diffracted waves. In a first step, the grating coupler was simplified to a $2 \mathrm{D}$ problem due to the high difference between the waveguide width and the heigh dimensions. The results demonstrated that the optimum waveguide width is around $14 \mu \mathrm{m}$ width, for the SM2000 fiber (11 um core diameter). Also, the grating period and filling factor were optimized, obtaining values of $630 \mathrm{~nm}$ and 0.5 , respectively. However, in a second step, several waveguide widths (10 $\mu \mathrm{m}$ to $15 \mu \mathrm{m})$ using 3D Finite Difference Time Domain method (3D-FDTD) were analysed, in order to study the effect on the misalignments during the fiber to grating coupling on the coupling efficiency. The results evidenced the best performance for the $15 \mu \mathrm{m}$ width in the case of the $\mathrm{TE}_{0}-\mathrm{LP}_{01}$ and $\mathrm{TE}_{1}-\mathrm{LP}_{11}$ modes, with a coupling efficiency around $49.7 \%$ and $46.3 \%$, respectively. The grating couplers with $12 \mu \mathrm{m}$ and $14 \mu \mathrm{m}$ waveguide widths were fabricated. The $14 \mu \mathrm{m}$ width was fabricated since it shows the best compromise when the integrated device size is considered, whilst the $12 \mu \mathrm{m}$ width was chosen because is the standardized grating coupler for the $\mathrm{TE}_{0^{-}}$ $\mathrm{LP}_{01}$ mode coupling. The experimental results achieved a perfect Gaussian profile (modal purity of $100 \%$ ) for the $L P_{01}$ mode, and the two $L P_{11}$ modes evidenced a modal purity of $89 \%$ for the $12 \mu \mathrm{m}$ width and $92 \%$ for the $14 \mu \mathrm{m}$ width. Also, the insertion losses for the $\mathrm{LP}_{01}$ mode were $9 \mathrm{~dB}$ and $8.2 \mathrm{~dB}$, and for the $\mathrm{LP}_{11}$ were $18 \mathrm{~dB}$ and $16 \mathrm{~dB}$, respectively.

Finally, the grating coupler with a $14 \mu \mathrm{m}$ width obtained a $49 \% \mathrm{LP}_{01}-\mathrm{TE}_{0}$ efficiency with a $5 \mu \mathrm{m}$ alignment tolerance, whereas the $\mathrm{LP}_{11}-\mathrm{TE}_{1}$ efficiency is $45 \%$ with a $2.6 \mu \mathrm{m}$ alignment tolerance. The purity achieved was $100 \%$ and $90 \%$, respectively. 


\section{CHAPTER 6. CONCLUSION AND FURTHER WORK}

\subsection{Main conclusions}

This PhD thesis covers the study of the mode division multiplexing in some specific scenarios, which are interesting for both the industry and the scientific community devoted to the design and manufacturing of fiber-based or integrated devices in optical transmission media. In fact, there are some gaps in the mode division multiplexing literature that this works attempts to fulfill. According to this, several different topics are treated throughout this work, with the mode division multiplexing as the common nexus to all of them. Despite the particularities of each case, the treatment given for the analysis of the mode coupling breakdown is very similar. The theoretical analysis of the different scenarios is based on the mode coupling theory. This constitutes the basic theory to understand the coupling between the same or higher modes in different structures, such as the waveguide or the optical fiber. After that, a simulation analysis based on the three-dimensional beam (3D) propagation method (3D-BPM) and the 3D Finite Difference Time Domain method (3D-FDTD) is employed in order to achieve a deeper knowledge of the mode coupling. Both analysis constitute a great aid to understand the coupling between the different modes in the different structures of the considered problem. Although the contents of this Ph.D. thesis are mainly focused on the numerical and simulation analysis, in most of the treated scenarios experimental measurements were carried out in order to assess the presented simulation frame.

The content of this Ph.D. thesis can be splitted into two different thematic areas: mode division multiplexing based on optical fibers using a standard single-mode fiber at $850 \mathrm{~nm}$, and mode division multiplexing in integrated devices based on SOI technology at $1550 \mathrm{~nm}$. For each of these topics, relevant and novel conclusions of the present study can be extracted. Next, the most remarkable findings will be outlined.

The mode division multiplexing analysis performed at $850 \mathrm{~nm}$ for optical fibers revealed the capability to increase the optical transmission using low cost devices. The first study is focused on the design of two different optical couplers (symmetric and asymmetric). With the symmetric coupler a mode converter is required to convert the $L P_{01}$ mode into $L P_{11}$ mode, however, the precision requirement during the fabrication process is low compared with the asymmetric coupler. In the latter case, the fiber is pre-tapered during the fabrication of the coupler and, therefore, a high alignment precision is required. However, no additional elements are needed to achieve the mode conversion. A second study related with the mode division multiplexing at $850 \mathrm{~nm}$ is based on the design of a mechanical mode converter using symmetrical couplers. For this case, it was considered a standard single-mode fiber with a $250 \mu \mathrm{m}$ cladding and no additional coating. This point is relevant for the correct conversion in order to apply pressure with a grating, generating an asymmetric perturbation in the optical fiber. However, a mode stripper is placed after each VCSEL in order to eliminate any residual $\mathrm{LP}_{11}$ content without reducing the $\mathrm{LP}_{01}$ power. Additionally, the mode converter can be tuned in a great bandwidth with the advantage of adapting it to tolerances in fiber specifications or laser performance. This statement was also experimentally verified in a $1 \mathrm{~km}$ fiber transmission test. Finally, the third study point out the possibility of using commercially available coupler devices based on SMF-28 and designed for $1550 \mathrm{~nm}$ operation. Different optical couplers (with distinct coupling ratios) have been considered. An experimental test campaign was carried out to validate the simulation results, demonstrating the feasibility of using commercially optical couplers at $850 \mathrm{~nm}$ operation and designed for $1550 \mathrm{~nm}$. In this way, it is possible to use low cost devices with no extra mode strippers, mode converters or mode rotators for mode multiplexing and demultiplexing.

With regard to the mode division multiplexing analysis at $1550 \mathrm{~nm}$ in integrated devices based on SOI technology, optimization of the different devices in order to increase the robustness to fabrication tolerances has been presented. The first study is focused on the optimization of the strip structure acting as an asymmetrical directional coupler. The main parameter analyzed was the gap between the waveguides, based on the effective refractive indexes in the mode 
coupling theory, and a good agreement was found when the results were compared with the proper waveguide simulations of the whole devices performed with the commercial software RSoft 3D-BPM. As a result, the optimization of the ADC design allows to increase the fabrication tolerances in comparison with the state-of-the-art. An experimental test was carried out corroborating the simulation results. The second study presents a new method of analysis based on the even and odd supermodes. This method is revealed as an alternative to the common analysis based on the effective refractive indexes of each individual waveguide, offering an extra point of view. The analysis of the width, gap, height and slab thickness allows to determine the most critical parameter during the fabrication. Additionally, the ridge structure was analyzed in comparison with the strip structure obtaining a high yield. Finally, the third study treats the analysis of several grating coupler designs with the aim to find the optimum $L P_{01}-T E_{0}$ and $L P_{11}-T E_{1}$ coupling. Until now, only the $L P_{01}-T E_{0}$ coupling has been employed with the standardized grating coupler parameters. This new study offers the possibility to achieve an improved design for the simultaneous coupling of both modes pairs and to optimize the alignment tolerances. Moreover, the 3D Finite Difference Time Domain method (3D-FDTD) revealed significant results that were corroborated experimentally in the test measurements.

\subsection{Further work}

As a result of the work performed throughout this Ph.D. thesis, it has been achieved a deeper understanding of several specific scenarios related with the mode division multiplexing in the $850 \mathrm{~nm}$ and $1550 \mathrm{~nm}$ bands. Thus, the conclusions extracted are of interest for the design of many types of mode converters and multiplexers/demultiplexers devoted to MDM systems. However, it is evident that the presented studies do not entirely cover all the possible cases, and further investigation of these topics will lead to the appearance of novel results. Next, we propose briefly some topics that could be of interest as future research lines.

- Study of bimodal propagation at $850 \mathrm{~nm}$ using IEEE standards: adaptation of the LP 01 and $\mathrm{LP}_{11}$ modes propagation for short range optical data links to the current IEEE standards.

- Study of new integrated converter structures: it is proposed to extend the study of the asymmetrical directional couplers to other type of structures such as tapers, $\mathrm{Y}-$ junctions, etc.

- Study of a new design of grating coupler for TMF based on apodization: in this Ph.D. thesis, the new design of grating coupler for the simultaneous coupling of the both $\mathrm{LP}_{01}$ and $\mathrm{LP}_{11}$ modes was restricted to the periodic structure. In this kind of structure only can be obtain a maximum of $50 \%$ coupling efficiency for both modes. Hence, there are other types of structure such as apodized gratings that it can achieve a higher coupling efficiency. It would be necessary to find a compromise between the best coupler design for the $T E_{0}-\mathrm{LP}_{01}$ design and the $\mathrm{TE}_{1}-\mathrm{LP}_{11}$ design. 


\section{ANNeX A. Publications}

\section{A.1 International peer-reviewed journals}

[Cor16] J. L. Corral, D. Garcia-Rodriguez, R. Llorente, "Mode-selective couplers for Two-Mode Transmission at $850 \mathrm{~nm}$ in Standard SMF," Photon. Technol. Lett. 28(4), 425-428 (2016).

[Gar17a] D. Garcia-Rodriguez, J. L. Corral, and R. Llorente, "Mode Conversion for Mode Division Multiplexing at $850 \mathrm{~nm}$ in Standard SMF," Photon. Technol. Lett. 29(11), 929-932 (2017).

[Gar 17b] D. Garcia-Rodriguez, J. L. Corral, A. Griol, and R. Llorente, "Dimensional variation tolerant mode converter/multiplexer fabricated in SOI technology for two-mode transmission at $1550 \mathrm{~nm}, "$ Opt. Lett. 42(7), 1221-1224 (2017).

[Gar 18a] D. Garcia-Rodriguez, J. L. Corral, and R. Llorente, "Design of Asymmetrical Directional Couplers on Ridge and Strip SOI technology with high-dimensional variation tolerance," Opt. Lett. 43(11), 2491-2494 (2018).

[Gar 18b] D. Garcia-Rodriguez, J. L. Corral, A. Griol, and R. Llorente, "Bimodal grating coupler design on SOI technology for mode division multiplexing at $1550 \mathrm{~nm}$," Opt. Exp. 26(15), 19445-19455 (2018).

\section{A. 2 International conferences}

[Llo14] Roberto Llorente, Maria Morant, David Garcia-Rodriguez, Pau Medina, Juan Luis Corral and Vicenç Almenar, "Few-Mode Optical Transmission Systems in the Visible Band", Invited paper in International Conference on Transparent Optical Networks, ICTON2014, Graz, Austria, 6-10 July 2014.

[Llo15] Roberto Llorente, Maria Morant, Andres Macho, David Garcia-Rodriguez and Juan Luis Corral, "Demonstration of a Spatially Multiplexed Multicore Fibre-Based Next-Generation Radio-Access Cellular Network", Invited paper in International Conference on Transparent Optical Networks, ICTON2015, Budapest, Hungria, 5-9 July 2015.

[Gar15] David Garcia-Rodriguez, Roberto Llorente, Juan L. Corral and Maria Morant, "Conversión Modal mediante Acopladores Pasivos para Sistemas de Comunicaciones Ópticas de Modos Limitados", 19th International Congress on Project Management and Engineering, AEIPRO 2015, Granada, España, 15-17 July 2015.

[Llo16] Roberto Llorente, Andres Macho, David Garcia-Rodriguez, Maria Morant and Juan Luis Corral, "Towards Multidimensional Multiplexing in Multicore Fiber Optical Data Links", Invited paper in International Conference on Transparent Optical Networks, ICTON2016, Trento, Italia, 10-14 July 2016.

[Gar16] David Garcia-Rodriguez, Juan L. Corral, Amadeu Griol and Roberto Llorente, "Silicon Photonic Integrated Mode Converter and Multiplexer for Few-Mode Fiber at 1550 nm", V Conferencia Española de Nanofotónica, CEN 2016, 20-22 June 2016.

[Gar17a] David Garcia-Rodriguez, Juan L. Corral, Amadeu Griol and Roberto Llorente, "Mode-converter and multiplexer based on SOI technology for few-mode fiber at $1550 \mathrm{~nm}$ ", Proc. Next-Generation Optical Communication: Components, Sub-Systems, and Systems VI, SPIE OPTO, 28 Jan-2 Feb 2017.

[Gar17b] David Garcia-Rodriguez, Maria Morant, Juan L. Corral and Roberto Llorente, "Mode Multiplexing and Demultiplexing by a Standard Single-Mode Coupler for $850 \mathrm{~nm}$ Few-Mode Transmission Systems", 43th European Conference and Exhibition on Optical Communication (ECOC2017), 17-21 September 2017.

[Llo17] Roberto Llorente, Maria Morant, David Garcia-Rodriguez and Juan Luis Corral, "Spatial division multiplexing in the short and medium range: From the datacenter to the fronthaul", 
Invited paper in International Conference on Transparent Optical Networks, ICTON2017, Girona, España, 2-6 July 2017. 


\section{References}

[ESS10] R.-J. Essiambre, G. Kramer, P. J. Winzer, G. J. Foschini, and B. Goebel, "Capacity limits of optical fiber networks," J. Lightwave Technol. 28(4), 662-701 (2010).

[RIC13] D. J. Richardson, J. M. Fini, and L. E. Nelson, "Space Division Multiplexing in Optical Fibers," Nat. Photonics 7(5), 354-362 (2013)

[MIZ16] T. Mizuno, H. Takara, A. Sano, and Y. Miyamoto, "Dense space-division multiplexing transmission systems using multi-core and multi-mode fiber," J. Lightwave Technol. 34(2), 582-592 (2016).

[RYF12] R. Ryf, S. Randel, A. Gnauck, C. Bolle, A. Sierra, S. Mumtaz, M. Esmaeelpour, E. Burrows, R. Essiambre, P. Winzer, D. Peckham, A. McCurdy, and R. Lingle, "Mode-division multiplexing over $96 \mathrm{~km}$ of few-mode fiber using coherent $6 \times 6$ MIMO processing," J. Lightwave Technol. 30(4), 521-531 (2012).

[SAS17] Y. Sasaki, K. Takenaga, S. Matsuo, K. Aikawa, K. Saitoh, "Few-mode Multicore Fibers for Long-haul Transmission Line", Optical Fiber Technology (ELSEVIER) 35, 19-27 (2017).

[MOR11] T. Mori, T. Sakamoto, T. Yamamoto, S. Tomita, "Coherent optical MIMO transmission over $20 \mathrm{~km}$ GI multi-mode fiber by using digital coherent receiver with mode convergence unit", Opt. Exp. 19(17), 16252-16258 (2011).

[FRA10] B. Franz, D. Suikat, R. Dischler, F. Buchali, and H. Buelow, "High speed OFDM data transmission over $5 \mathrm{~km}$ GI-multimode fiber using spatial multiplexing with $2 \times 4$ MIMO processing," Proc. European Conference on Optical Communication (ECOC'10), Tu.3.C.4 (2010).

[BER82] S. Berdague and P. Facq, "Mode division multiplexing in optical fibers," Appl. Opt. 21(11), 1950-1955 (1982).

[TAT15] J. A. Tatum, D. Gazula, L. A. Graham, J. K. Guenter, R. H. Johnson, J. King, C. Kocot, G. D. Landry, I. Lyubomirsky, A. N. MacInnes, E. M. Shaw, K. Balemarthy, R. Shubochkin, D. Vaidya, Y. Man, and F. Tang, "VCSEL-based interconnects for current and future data centers," J. Lightwave Technol. 33(4), 727-732 (2015).

[KAS13] M. Kasahara, K. Saitoh, T. Sakamoto, N. Hanzawa, T. Matsui, K. Tsujikawa, F. Yamamoto, and M. Koshiba, "Design of few-mode fibers for mode-division multiplexing transmission," IEEE Photonics J. 5(6), 7201207 (2013).

[HAN15] N. Hanzawa, K. Saitoh, T. Sakamoto, T. Matsui, K. Tsujikawa, T. Uematsu, and F. Yamamoto, "PLC-based four-mode multi/demultiplexer with $\mathrm{LP}_{11}$ mode rotator on one chip," J. Lightwave Technol. 33(6), 1161-1165 (2015).

[WAN14] J. Wang, S. He, and D. Dai, "On-chip silicon 8-channel hybrid (de)multiplexer enabling simultaneous mode- and polarization-division-multiplexing," Laser Photon. Rev. 8(2), L18-L22 (2014).

[CIS17] Cisco white paper, "Cisco Visual Networking Index: Forecast and Methodology, 2016-2021,"https://www.cisco.com/c/en/us/solutions/collateral/service-provider/visual-netw orking-index-vni/complete-white-paper-c11-481360.pdf

[SAK11] T. Sakamoto, T. Mori, T. Yamamoto, L. Ma, N. Hanzawa, S. Aozasa, K. Tsujikawa, and S. Tomita, "Transmission over large-core few-mode photonic crystal fiber using distanceindependent modal dispersion compensation technique," Opt. Express 19(26), B478-B485 (2011).

[VIS11] D. Visani, C. M. Okonkwo, Y. Shi, H. Yang, H. P. A. van den Boom, G. Tartarini, E. Tangdiongga, and A. M. J. Koonen, " $3 \times 2^{\mathrm{N}}-\mathrm{QAM}$ constellation formats for DMT over $1-\mathrm{mm}$ core diameter plastic optical fiber," IEEE Photon. Technol. Lett. 23(12), 768-770 (2011). 
[KRU11] R. Kruglov, S. Loquai, C. A. Bunge, O. Ziemann, B. Schmauss, and J. Vinogradov, "10 Gbit/s short-reach transmission over 35 m large-core graded-index polymer optical fiber", Optical Fiber Communication Conference and Exposition (OFC/NFOEC), 1-3 (2011).

[LLO14] R. Llorente, M. Morant, D. Garcia-Rodriguez, P. Medina, J. L. Corral and V. Almenar, "Few Mode Optical Transmission Systems in the Visible Band", Invited paper in International Conference on Transparent Optical Networks, ICTON2014, paper Th.A1.3.

[HAN11] N. Hanzawa, K. Saitoh, T. Sakamoto, T. Matsui, S. Tomita, and M. Koshiba, "Demonstration of mode-division multiplexing transmission over $10 \mathrm{~km}$ two-mode fiber with mode coupler," in Optical Fiber Communication Conference, OSA Technical Digest (CD) (Optical Society of America, 2011), paper OWA4.

[TIA12] Z. Tian, C. Chen, and D. V. Plant, "850-nm VCSEL transmission over standard singlemode fiber using fiber mode filter," IEEE Photon. Technol. Lett., 24(5), 368-370 (2012).

[MIL16] G. Milione, E. Ip, M. J. Li, J. Stone, G. Peng, and T. Wang, "Mode crosstalk matrix measurement of a $1 \mathrm{~km}$ elliptical core few-mode optical fiber," Opt. Lett. 41(12), 2755-2758 (2016).

[LI11] A. Li, A. Al Amin, X. Chen, and W. Shieh, "Transmission of 107-Gb/s mode and polarization multiplexed CO-OFDM signal over a two-mode fiber," Opt. Express 19(9), 88088814 (2011).

[DAI13] D. Dai, J. Wang, and Y. Shi, "Silicon mode (de)multiplexer enabling high capacity photonic networks-on-chip with a single-wavelength-carrier light," Opt. Lett. 38(9), 14221424 (2013).

[SUN16] C. Sun, Y. Yu, M. Ye, G. Chen, and X. Zhang, "An ultra-low crosstalk and broadband two-mode (de)multiplexer based on adiabatic couplers," Sci. Rep. 6, 38494 (2016).

[ZAN16] A. Zanzi, A. Brimont, A. Griol, P. Sanchis, and J. Marti, "Compact and low-loss asymmetrical multimode interference splitter for power monitoring applications," Opt. Lett. 41(2), 227-229 (2016).

[ZHA15] Z. Zhang, X. Hu, and J. Wang, "On-chip optical mode exchange using tapered directional coupler," Sci. Rep. 5, 16072 (2015).

[XIN13] J. Xing, Z. Li, X. Xiao, J. Yu, and Y. Yu, "Two-mode multiplexer and demultiplexer based on adiabatic couplers," Opt. Lett. 38(17), 3468-3470 (2013).

[DIN13] Y. Ding, J. Xu, F. Da Ros, B. Huang, H. Ou, and C. Peucheret, "On-chip two-mode division multiplexing using tapered directional coupler-based mode multiplexer and demultiplexer," Opt. Express 21(8), 10376-10382 (2013).

[HAN14] N. Hanzawa, K. Saitoh, T. Sakamoto, T. Matsui, K. Tsujikawa, M. Koshiba, and F. Yamamoto, "Mode multi/demultiplexing with parallel waveguide for mode division multiplexed transmission," Opt. Express 22(24), 29321-29330 (2014).

[LIU07] J. M. Liu, Photonic Devices (Cambridge University, 2007).

[VEN96] A. M. Vengsarkar, P. J. Lemaire, J. B. Judkins, V. Bhatia, T. Erdogan, and J. E. Sipe, "Long-period fiber gratings as band-rejection filters," J. Lightwave Technol. 14(1), 58-65 (1996).

[GIL12] I. Giles, A. Obeysekara, R. Chen, D. Giles, F. Poletti, and D. Richardson, "Fiber LPG mode converters and mode selection technique for multimode SDM," IEEE Photonics Technology Letters 24, 1922-1925 (2012).

[GAL08] J. V. Galan, P. Sanchis, J. Blasco, and J. Marti, "Study of High Efficiency Grating Couplers for Silicon-Based Horizontal Slot Waveguides," IEEE Photonics Technol. Lett. 20(12), 985-987 (2008). 
[ROE06] G. Roelkens, D. Van Thourhout, and R. Baets, "High efficiency Silicon-on-Insulator grating coupler based on a poly-Silicon overlay," Opt. Express 14(24), 11622-11630 (2006).

[TAI05] D. Taillaert, "Grating couplers as Interface between Optical Fibres and Nanophotonic Waveguides," PhD Dissertation, Universiteit Gent, 2005.

[RSOFT] https://www.synopsys.com/optical-solutions/rsoft/rsoft-products.html

[LI12] A. Li, X. Chen, A. Al Amin, and W. Shieh, "Fused fiber mode couplers for few-mode transmission," IEEE Photon. Technol. Lett. 24(21), 1953-1956 (2012).

[ISM14] R. Ismaeel, T. Lee, B. Oduro, Y. Jung, and G. Brambilla, "All-fiber fused directional coupler for highly efficient spatial mode conversion," Opt. Express 22(10), 11610-11619 (2014).

[Li13] A. Li, J. Ye, X. Chen, and W. Shieh, "Fabrication of a low-loss fused fiber spatial-mode coupler for few-mode transmission," IEEE Photon. Technol. Lett. 20, 1985-1988 (2013).

[MO005] S. Moon and D. Y. Kim, "Effective single-mode transmission at wavelengths shorter than the cutoff wavelength of an optical fiber," IEEE Photon. Technol. Lett. 17, 2604-2606 (2005)

[MIL15] G. Milione, M. P. J. Lavery, H. Huang, Y. Ren, G. Xie, T. A. Nguyen, E. Karimi, L. Marrucci, D. A. Nolan, R. R. Alfano, and A. E. Willner, " $4 \times 20 \mathrm{Gbit} / \mathrm{s}$ mode division multiplexing over free space using vector modes and a q-plate mode (de)multiplexer," Opt. Lett. 40(9), 1980-1983 (2015).

[PRA15] P. Pradhan, D. Sengupta, H. Rehouma, V. Francois, C. Tremblay, and B. Ung, "Excitation of vector modes in few-mode fiber using wire-based mechanical long period fiber grating," Proc. Photonics North, 1 (2015).

[XU14] D.-X. Xu, J. H. Schmid, G. T. Reed, G. Z. Mashanovich, D. J. Thomson, M. Nedeljkovic, X. Chen, D. Van Thourhout, S. Keyvaninia, and S. K. Selvaraja, "Silicon photonic integration platform Have we found the sweet spot," IEEE J. Sel. Top. Quantum Electron. 20(4), 189-205 (2014).

[GAR17] D. Garcia-Rodriguez, J. L. Corral, A. Griol, and R. Llorente, "Dimensional variation tolerant mode converter/multiplexer fabricated in SOI technology for two-mode transmission at $1550 \mathrm{~nm}$," Opt. Lett. 42(7), 1221-1224 (2017).

[PAN16] C. Pan and B. M. A. Rahman, "Accurate Analysis of the Mode (de)multiplexer Using Asymmetric Directional Coupler," J. Lightwave Technol. 34(9), 2288-2296 (2016).

[MIK14] J. C. Mikkelsen, W. D. Sacher, and J. K.-S. Poon, "Adiabatically widened silicon microrings for improved variation tolerance," Opt. Express 22(8), 9659-9666 (2014).

[ANT11] M. Antelius, K. B. Gylfason, and H. Sohlström, "An apodized SOI waveguide-to-fiber surface grating coupler for single lithography silicon photonics," Opt. Express 19(4), 35923598 (2011).

[VAN07] F. Van Laere, G. Roelkens, M. Ayre, J. Schrauwen, D. Taillaert, D. Van Thourhout, T. F. Krauss, and R. Baets, "Compact and highly efficient grating couplers between optical fiber and nanophotonic waveguides," J. Lightwave Technol. 25, 151-156 (2007).

[WOH16] B. Wohlfeil, G. Rademacher, C. Stamatiadis, K. Voigt, L. Zimmermann, and K. Petermann, "A Two-Dimensional Fiber Grating Coupler on SOI for Mode Division Multiplexing," IEEE Photonics Technol. Lett. 28(11), 1241-1244 (2016). 